\title{
Ondes et radiations électromagnétiques et gravitationelles en relativité générale.
}

\author{
Memoire de Andrí Lichnerowicz (Paris)
}

A Antonio Signorini pour son $70^{m e}$ anniversaire.

\begin{abstract}
Résụmé. - Théorie des ondes et radiations gravitationelles basée sur l'analogie existant entre les comportements au tenseur de courbure et du tenseur champ électromagnétique en relativité générale. Contribution à la quantification des champs.
\end{abstract}

\section{INTRODUCTION}

L'un des problèmes le plus importants de la théorie relativiste de la gravitation concerne la définition et les propriétés des ondes et radiations gravitationnelles. Le problème a fait l'objet, an cours des dernières années, de nombreux et intéressants travanx.

Dans le cadre de la relativité restreinte une théorie classique satisfaisante des ondes et radiations éleotromagnétiques a pu être élaborée et il m'a semblé de bonne méthode de la développer dans le cadre de la relativité générale, de façon à l'adapter aisément au cas gravitationnel. Il apparaît que, dans ce cas, c' est le tenseur de courbure qui doit jouer le rôle essentiel, au point de vae mathématique comme au point de vue physique, et ceci se trouve en plein accord avec le point de vue de Pirani [1, 2]. Pour une métrique satisfaisant aux équations d'EINstein $R_{\alpha \beta}=0$, le tenseur de courbure satisfait à deux groupes de relations dont la ressemblance formelle avec les equations de MAXwell da vide est frappante, soit:

$$
S \nabla_{Y} R_{x \beta, \lambda \mu}=0 \quad \nabla_{\alpha} R_{\beta, \lambda \mu}=0
$$

où $\nabla_{\alpha}$ est l'opérateur de dérivation covariante et où $S$ indique une somma. tion après permutation circulaire sur les trois indices $\alpha, \beta, \gamma$, Ce sont ces relations qut jouent un rôle fondamental dans la partie gravitationnelle de ce travail.

Le chapitre I est consacré à la théorie des ondes et radiations électromagnétiques en relativité générale. Après avoir rappelé des résultats classiques, la notion de 2-forme singulière est dégagée et appliquée à la définition de la notion de radiation électromagnétique pure comme à l'étude des discontinuités du tenseur dérivé du champ électromagnétique. Dans ce cas, on voit 
apparaitre de manière naturelle aux points du front d'onde un tenseur d'ordre 4 conservatif.

Dans le chapitre II sont étudiées les discontinuités du tenseur de courbure. Cette étude conduit ì distinguer, parmi les tenseurs $H_{\alpha \beta, \lambda \mu}$ admettant le type de symétrie du tensear de courbure, ceux correspondant à ce qu'on pourrait nommer une «double 2-forme singulière». Tres conditions correspondantes entrainent pour le tenseur contracté $H_{\alpha \beta}$ une forme remarquable. $L$ analyse des relations differentielle (1) anx quelles sont soumises les discontinuités du tenseur de courbure dans le cas $B_{\alpha \beta}=0$ conduit à des identités de conservation pour un tenseur d'ordre 4. Il en est encore de même s'il y a simultanément discontinuité du tenseur de courbure et discontinuité $d u$ tenseur dérivé d'un champ électromagnétique satisfaisant aux équations de MaXWell da vide $\left({ }^{2}\right)$.

Dans le chapitre III, j' indique une définition des notions de radiation totale et radiation gravitationnelle pure, notions qui correspondent à un cas particulier remarquable de la classification de Patrov-Pinanr. Le champ de vecteurs isotropes mis en évidence admet pour trajectoires des géodesiques de longueur nulle. Gracé a un travail de Bond, perfectionnant un exemple de Rosen, on peut construire des exemples effectifs de telles radiations. Dans la cas oủ $R_{x_{\beta}}=0$ (ou plus généralement $\lambda g_{\alpha_{\beta}}$ ), j'étudie les propriétés d'un tenseur d'ordre 4 introduit, en accord avec mon point de vue, par L. BEL [2]. Ces propriétés sont formellement très semblables ì celles du tenseur de MAXwELi d'un champ électromagnétique satisfaisant aux équatiaes de MAXweLL du vide et il semble qu ce tenseur appelle de nouvelles et importantes recherches.

Le chapitre IV est consacré au comportement de l'accélération relative de deux particules d'essai voisines relativement à une onde ou radiation, selon un point de vue développé par Sxnge et par Pirani [2]. L'étude du cas des particules chargées en présence d'un champ électromaguetique met en évidence une difference de comportement pour une onde gravitationnelle et une onde électromagnétique.

Dans le chapitre $V$, j'ai adopté le cadre pentadimensionnel pour traduire radiation electromagnétique et radiation gravitationnelle dans un même formalisme, ce qui conduit à «tronquer» le tenseur de courbure de la varieté pentadimensionnelle.

Au chapitre VI, j'exploite enfin les analogies signalées entre gravitation et électromagnétisme pour développer des processus de quantification parallèles $d n$ champ électromagnétique en relativité restreinte et du champ gravi. tationnel a l'approximation linéaire. Dans le premier eas, la quantification.

(1) Relations clonnées indépendamment par TraUTMax et par l'auteur. 
s'effectue directement sur le tenseur champ électromagnétique et conduit naturellement à la représentation classique da photon, dans le second cas elle s'effectue sur le tenseur de courbure et conduit, comme je le montre, a une représentation equivalente à celle du graviton. Il semble cependant que ce processus de quantification soit plus satisfaisant, à la fois mathema* tiquement et physiquement, que celui mis en jeu par la théorie classique du graviton. Les équations écrites au début. de cette introduction apparaissent bien comme les équations fondamentales de champ, la condition $R_{\alpha \beta}=0$ se présentant alors comme un simple condition initiale.

\section{I. - Ondes et radiations électromagnétiques en relativité générale.}

\section{Généralités.}

Dans toute théorie relativiste du champ gravitationnel, l'élément primitif est constitué par une variété «espace-temps $V_{4}$ de dimensions 4., donée d'une structure de variété différentiable qu'il est essentiel de préciser: pour des raisons étroitement liées à la covariance du formalisme et qui apparaissent par l'analyse des équations du champ gravitationnel (voir $\$ 17-18$ ), nous sommes amenés à supposer que dans l'intersection des domaines de deux systèmes de coordonnées locales admissibles, les coordonnées locales d'un point dans l'un des systèmes sont des fonctions 4 fois dérivables, à jacobien non nul, des coordonnées de ce point dans l'autre système, les dérivées premières et secondes étant continues, les dérivées troisièmes étant seulement continues par morceanx. Nous traduisons ceci en disant que la variété $V_{4}$ est de classe $\left(O^{2}, C^{4}\right.$ par morceaux). Sauf avis contraire, $V_{4}$ est supposé orientable.

Sur $V_{4}$ est définie une métrique riemannienne $d s^{2}$, de type hyperbolique normal, à un carré positif et 3 carrés négatifs. L' expression locale de cette métrique dans un système de coordonnées admissibles est:

$$
d s^{2}=g_{\alpha \beta} d x^{\alpha} d x^{e} \quad(\alpha, \beta, \text { tout indice grec }=0,1,2,3) .
$$

Le tenseur métrique $g_{\alpha \beta}$, ou tenseur de gravitation, est supposé exactement $\left(C^{3}, C^{3}\right.$ par morceaux) ce qui est strictement compatible avec la structure différentiable imposée à $V_{4}$.

L'équation $d s^{2}=0$ définit en chaque point $x$ de $V_{4}$, un cône réel $C_{x}$, le cône élémentaire en $x$. Son intérienr et son extérieur définissent respectivement pour une direction l'orientation dans le temps et l'orientation dans l'espace. Un vecteur tangent a $V_{4}$ est dit normé si le module de son carré est égal à 1 . Un repère orthonormé au point $x$ de $V_{4}$ est un ensemble ordonné de 4 vecteurs $\left(\vec{e}_{\alpha}\right)$ en $x$ tels que

$$
\overrightarrow{e_{\alpha}} \cdot \overrightarrow{e_{\beta}}=0 \text { pour } \alpha \neq \beta \quad{\overrightarrow{e_{0}}}^{2}=1 \quad \vec{e}_{u}^{2}=-1 \quad(u=1,2,3)
$$


$\vec{e}_{0}$ définit une direction de temps et le 3 -plan perpendiculaire défini par les $\vec{e}_{u}$ est dit l'espace associé à cette direction de temps. Nous rappelons qu' un 2-plan ou un 3-plan est dit orienté dans l'espace si toutes ses direc. tions sont orientées dans l'espace; il est orienté dans le temps dans le cas contraire.

Si un voisinage $U$ de $V_{4}$ est muni de repéres orthonormés, le $d s^{2}$ pent s'écrire localement

$$
d s^{2}=\left(\theta^{0}\right)^{2}-\sum_{w}\left(\theta^{u}\right)^{2}
$$

où les $\theta^{x}$ sont des formes de PFafr locales linéairement indépendantes.

Naturellement $V_{4}$ peut etre l'espace-temps de Minkowski de la relativité restreinte. Un repère orthonormé est alors an repère galiléen ordinaire (avec $c=1$ ). Dans le cas riemannien, la métrique définit sur l'espace vectoriel tangent au point $x$ de $V_{4}$, une structure d'espace de Minkowski. L'interprétation physique d'un tenseur défini au point $x$ de $V_{4}$ se déduit immediatement de la considération de cet espace de Mrnkowski tangent: rapporté ¿̀ un repère orthonormé, cet espace doit ètre identifié à l'espace-temps de la relativité restreinte rapporté a un repére galiléen, ce qui fournit directement, en termes de temps et d'espace associés à ce repère, les interprétations physiques désirées.

\section{Le champ électromagnétique en l'absence d'induction.}

Sur un domaine de $V_{4}$, et en l'absence de tout phénomène d'induction, le champ électromagnétique est représenté par un champ de tenseurs antisymétriques $F_{x \beta}$, de classe $\left(C^{0}, C^{2}\right.$ par morceaux). A ce tenseur on pent associer la 2-forme

$$
F=\frac{1}{2} F_{\alpha \beta} \theta^{\alpha} \wedge \theta^{\beta}
$$

Si $\eta_{x \beta \gamma \delta}$ est le tenseur elément de volume de la variété riemannienne $V_{4}$, on peut dédnire de $F$ le tenseur antisymétrique «adjoint» $(* F)_{x \beta}$ défini par:

$$
(* F)_{\alpha \beta}=\frac{1}{2} \eta_{\alpha \beta \gamma \delta} F r^{\delta} \text {. }
$$

Nous désignerons par $* F$ la 2 - forme associée, dite l'adjointe de $F$. On notera que, le discriminant $\mathrm{du} d s^{2}$ étant négatif $* * F=-F$.

Par rapport à un repére orthonormé $\left(\overrightarrow{e_{\alpha}}\right)$ en $x$, l'interprétation physique de $F$ et $* F$ est fournie par la règle suivante: si $\vec{u}=\overrightarrow{e_{0}}$, les vecteurs champ électrique et champ magnétique relatifs au temps et à l'espace définis par le èrerep sont les vecteurs d'espace (c' est-à-dire les vecteurs orthogonaux à $\vec{u}$ ) déterminés par

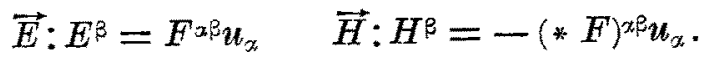


Si $(X, Y, Z)$ et $(L, M, N)$ sont les composantes respectives de $\vec{E}$ et $\vec{H}$ par rapport an repere $\left(\overrightarrow{e_{\alpha}}\right)$, on a le tableau:

$$
\left\{\begin{array} { l } 
{ X = F ^ { 0 1 } = F _ { 1 0 } = ( * F ) _ { 2 3 } = ( * F ) ^ { 2 3 } } \\
{ Y = F ^ { 0 2 } = F _ { 2 0 } = ( * F ) _ { 3 1 } = ( * F ) ^ { 3 1 } } \\
{ Z = F ^ { 0 3 } = F _ { 3 0 } = ( * F ) _ { 1 2 } = ( * F ) ^ { 1 2 } }
\end{array} \quad \left\{\begin{array}{l}
L=(* F)^{10}=(* F)_{01}=F_{23}=F^{23} \\
M=(* F)^{20}=(* F)_{02}=F_{31}=F^{31} \\
N=(* F)^{30}=(* F)_{03}=F_{12}=F^{12}
\end{array}\right.\right.
$$

On notera que, comme dans le repère $\left(e_{\alpha}\right) g_{00}=1, g_{u u}=-1$, la montée on la descente d'un indice d'espace $u$ s'effectue areo changement de signe, alors $q \mathbf{u}^{\prime}$ il n'en est pas de même pour l'indice temporel 0. An tenseur champ électromagnétique on peut attacher deux scalaires intéressants: les produits scalaires de la forme $F$ par elle-même ou par la forme $* F$, soit:

$$
\Psi=(F, F)=\frac{1}{2} F_{\alpha \beta} F^{\alpha \beta} \quad \Phi=(F, * F)=\frac{1}{2} F_{\alpha \beta \beta}(* F)^{\alpha \beta}
$$

$\Phi=0$ exprime que la forme $F$, et par suite la forme $* F$, sont de rang infé. rieur à 4. Dans un repère orthonormé, les scalaires $\Phi$ et $\Psi$ ont pour expressions :

$$
\Psi=L^{2}+M^{2}+N^{2}-X^{2}-Y^{2}-Z^{2} \quad \Phi=2(L X+M Y+N Z)
$$

soit, les carrés et produits scalaires étant évalués à l'aide de la métrique d'espace définie positive:

$$
\Psi=\vec{H}^{2}-\vec{E}^{2} \quad \Phi=2 \vec{E} \cdot \vec{H}
$$

et les seconds membres sont des invariants d'espace-temps.

\section{Tenseur de Maxwell et vecteur de Poynting.}

L'étude de la relativité restreinte a conduit à introduire comme tenseur d'impulsion-énergie du champ électromagnétique le tenseur de MaxwELL défini par:

$$
\tau_{\alpha \beta}=\frac{1}{4} g_{\alpha \beta} F_{\lambda \mu} F^{\lambda \mu}-F_{\alpha \beta} F_{\beta}^{\rho} \quad\left(\tau_{\alpha}^{\alpha}=0\right)
$$

Lorsqu'on rapporte le champ electromagnétique à un repère orthonormé $\left(\overrightarrow{e_{a}}\right)$, on voit que ce tenseur de MaxwrLL est construit avec le tenseur d'espace $\left(\tau_{u v}\right)$ des tensions électromagnétiques le vecteur d'espace de Poynting de composantes :

$$
P_{0}=0 \quad P_{u}=\tau_{0 u}
$$

et l'énergie du ehamp électromagnétique

$$
\tau_{00}=\frac{1}{2}\left(E^{2}+H^{2}\right)
$$


Si $n^{n}$ est un vecteur normé d'espace, le flux d'énergie électromagnétique à travers un élément de 2-surface orthogonal à $n^{u}$ de l'espace est proportionnel à $P_{u} n^{\prime}$. Pour que, quel que soit cet élément de surface, ce flux soit nul, il faut et il suffit que le vecteur de Porntring correspondant à $\vec{e}_{0}$ soit nul. Or si $\vec{u}=\overrightarrow{e_{0}}$, le vecteur de Poynting correspondant peut s'écrire:

$$
P_{z}=\left(g_{x} \curlyvee-u_{x} u r\right) \tau_{\beta \gamma} u^{\beta}
$$

Pour que $P_{x}$ soit nul, il faut et il suffit que

$$
\tau_{x \beta} u^{\beta}=\left(\tau_{\beta \gamma} u^{\beta} u Y\right) u_{\alpha}
$$

$c^{\circ}$ est-h̀-dire que $\vec{u}$ soit vecteur propre du tenseur de Maxwell par rapport an tenseur métrique. On a été ainsi conduit à étudier les vecteurs propres de $\tau_{\not \beta}$ par rapport à $g_{x \beta}$.

\section{Les équations du champ électromagnétique.}

a) Le champ électromagnétique satisfait aux équations de Maxwelt, dont le premier groupe exprime que $F$ dérive localement d'un potentielvecteur et $l e$ second relie $F$ aux sources de ce champ, c' est-ì-dire au vecteurcourant élecrtique. Ces équations peuvent s'écrire:

$$
E^{x} \equiv \frac{1}{2} \eta^{\beta \gamma \delta x} \nabla_{\beta} F_{Y \delta}=0 \quad D^{x} \equiv \nabla_{\beta} F^{\beta x}=J^{x}
$$

où $\nabla$ est l'opérateur de dérivation covariante dans la connexion riemannienne et $J^{x}$ le vecteur-courant électrique. De ces équations, il résulte par un calcul classique:

$$
\nabla_{\alpha} \tau_{\beta}^{x_{2}}=F_{\rho p} J^{p} \text {. }
$$

Je me placerai généralemeut ioi dans le cas purement électromagnétique: dans le domaine envisagé de $V_{4}$, le vecteur courant électrique est nul et le champ électromagnétique contribue seul au tensenr d'impulsion-énergie. Si $S_{x \beta}=R_{x_{\beta}}-(1 / 2) g_{\alpha \beta} R$ est le tenseur d'Einstein de la métrique, champ gravitationnel et champ électromagnétique sont liés par les équations d' Einstern:

$$
S_{\alpha \beta}=\chi \tau_{\alpha \beta} \text {. }
$$

De la nullité du courant, il résult d'aprés (4-2):

$$
\nabla_{x} \tau_{\beta}=0
$$

Cela résulte aussi des équations d'Enssteis (4-3) puisque le tenseur $S_{\alpha \beta}$ satisfait aux identités de conservation. Il y a ainsi compatibilité entre (4-3) et la condition de nullité du courant.

Soit $d$ l'operateur de différentiation extérieure sur les formes $\left(d^{2}=0\right)$, $\delta$ 
A. Lrchnerowicz: Ondes et radiations électromagnétiques, etc.

l'opérateur de codifférentiation défini sur une forme de degré $p$ par $\delta=(-1)_{*}^{p_{-1}} d *\left(\delta^{2}=0\right)$. Les équations de MaxwELL peuvent s' ecrire :

$$
d F=0 \quad \text { ou } \quad \delta(* F)=0
$$

et dans le cas purement électromagnétique:

$$
d(* F)=0 \quad \text { ou } \quad \delta F=0 .
$$

b) Dans cette section, nous ne nous servirons pas des equations d' EINstern. Remarquons seulement que si l'on substitue au champ électromagnétique pur un fluide matériel sans pression, au tenseur $\tau_{\alpha \beta}$ se trouve substitué le tenseur d'impulsion-énergie

$$
T_{\alpha \beta}=\rho u_{\alpha} u_{\rho}=\left(\sqrt{\rho} u_{\alpha}\right)\left(\sqrt{\rho} u_{p}\right)
$$

où $\rho$ est la densité de matière propre et $u_{\alpha}$ le vecteur-vitesse unitaire $\left.\overrightarrow{(u}^{2}=1\right)$ du fluide. Des conditions de conservation $\nabla_{\alpha} T_{\beta}^{\alpha_{\beta}}=0$ satisfaites par le tenseur d'impulsion-énergie, on déduit en vertu du caractère unitaire de $\vec{u}$

$$
\nabla_{\alpha}\left(\rho u^{\alpha}\right)=0
$$

et

$$
u^{*} \nabla_{\alpha} u^{\beta}=0
$$

(4-8) est l'équation de continuité d'après (4-9) les lignes de courant du fluide, trajectoires $d u$ champ de vecteurs $\vec{u}$, sont des géodésiques orientées dans le temps de la variété $V_{4}$.

\section{Valeurs propres du tenseur de Maxwell $\left({ }^{3}\right)$.}

L'étude de la nullité du vecteur de Pornting conduit à rechercher les valeurs et vecteurs propres de $\tau_{\alpha \beta}$ par rapport à $g_{\alpha \beta}$. Il s'agit ici d'une étude purement algébrique qui a lieu en un point déterminé de $\nabla_{4}$ et qui conduit a des expressions réduites, en repère orthonormé $\left(\vec{e}_{\alpha}\right)$ de $V_{4}$, pour le couple de formes $\left(F, * F^{\prime}\right)$. Dans ce paragraphe, $F$ pent être une 2-forme quelconque interprétée en termes de champ électromagnétique.

Donnons-nous arbitrairement le vecteur $\vec{e}_{0}$ de $\left(\vec{e}_{\alpha}\right)$ orienté dans le temps. Il lui correspond des vecteurs champ électrique et champ magnétique $\vec{E}$ et $\vec{H}$. Il est possible de choisir le 2-plan $\left(\overrightarrow{e_{2}}, \overrightarrow{e^{3}}\right)$ parallèle à la fois à $\vec{E}$ et $\vec{H}$. Le vectenr $e_{1}$ est alors fixé au signe près et l'on a $X=L=0$. Un repère orthonormé satisfaisant à cette condition sera dit un repère adapté à la forme $F$. Dans le 2-plan $\left(\overrightarrow{e_{2}}, \overrightarrow{e_{3}}\right)$ fixé, on peut alors choisir les vecteurs $\overrightarrow{e_{2}}$ et $\overrightarrow{e_{3}}$ de façon qu'ils soient vecteurs propres de la matrice $\left(\tau_{A B}\right)(A, B=2,3)$ par

(3) Nous résumons ici les résultats exposes dans LIohnerowacz Théories relativistes de la gravitation et de l'étectromagnétisme. Chap. 1. 
rapport a l'identité. On a alors $Y Z+M N=0$. Un repère adapté satisfaisant à cette condition sera dit simple pour $F$. Si l'on introduit deux nombres $\xi, \eta$ satisfaisant à

$$
Y^{2}+M^{2}=\xi^{2} \quad Z^{2}+N^{2}=\eta^{2} \quad Z M-Y N=\xi_{\eta}
$$

on démontre que, pour un repère simple, la matrice $\left(\tau_{\alpha \beta}\right)$ prend la forme

$$
\left(\tau_{\alpha \beta}\right)=\left(\begin{array}{cccc}
\frac{\xi^{2}+\eta^{2}}{2} & \xi \eta & 0 & 0 \\
\frac{\xi^{2}+\eta^{2}}{2} & 0 & 0 \\
& & \frac{\eta^{2}-\xi^{2}}{2} & 0 \\
& & & \frac{\xi^{2}-\eta^{2}}{2}
\end{array}\right) .
$$

Inversement si, relativement a un repère orthonormé, la matrice $\left(\tau_{\alpha \beta}\right)$ prend la forme:

$$
\left(\begin{array}{llll}
\tau_{00} & \tau_{01} & 0 & 0 \\
& \tau_{11} & 0 & 0 \\
& & \tau_{22} & 0 \\
& & & \tau_{33}=-\tau_{22}
\end{array}\right)
$$

on établit aisément que le repère est simple.

Cela posé, il est facile d'obtenir, à partir de (5-2), les valeurs propres de $\tau_{x \beta}$ par rapport à $g_{\alpha \beta}$. L'equation aux valeurs propres s'écrit:

Il en résulte:

$$
\left[s^{2}-\left(\frac{\xi^{2}-\eta^{2}}{2}\right)^{2}\right]^{2}=0
$$

THÉOREME. - Le tenseur de Maxwell d'un champ électromagnétique admet 4 valeurs propres réelles 2 à 2 égales et opposées, $\mathrm{k}, \mathrm{k},-\mathrm{k},-\mathrm{k}$.

On a posé

$$
4 k^{2}=\left(\xi^{2}-\eta^{2}\right)^{2}
$$

Il est aisé de relier $k$ aux invariants $\Psi$ et $\Phi$. On a $k^{2}=\left[\left(H^{2}-Z^{2}\right)-\left(N^{2}-Y^{2}\right)\right]^{2}=\left[M^{2}-Z^{2}+N^{2}-Y^{2}\right]^{2}-4\left(M^{2}-Z^{2}\right)\left(N^{2}-Y^{2}\right)$. Or de $(2 \cdot 6)$ et des conditions de simplicité il résulte: $\left[M^{2}+N^{2}-Y^{2}-Z^{2}\right]^{2}=\Psi^{2} \quad-4\left(M^{2}-Z^{2}\right)\left(N^{2}-Y^{2}\right)=4(M Y+N Z)^{2}=\Phi^{2}$ 
Ainsi :

$$
4 k^{2}=\Psi^{2}+\Phi^{2}
$$

\section{Le cas régulier.}

Il convient maitenant de distinguer le cas $k \neq 0$ et le cas $k=0$. Dans le premier cas nous dirons que la forme $F$ est régulière, dans le second $q u$ 'elle est singulière. Examinons le cas régulier; il existe alors deux valeurs propres distinctes $k$ et $-k$ et par suite deux 2-plans de vecteurs propres orthogonaux dont l'un est nécessairement orienté dans le temps et l'autre dans l'espace. Si $\overrightarrow{e_{\theta}}$ est un vecteur normé, orienté dans le temps, du premier 2-plan, $\overrightarrow{e_{3}}$ le vecteur normé orthogonal de ce 2-pla,n si $\overrightarrow{e_{1}}$ et $\overrightarrow{e_{2}}$ sont deux vecteurs orthogonaux normes du 2-plan orienté dans l'espace, le repére $\left(\overrightarrow{e_{\alpha}}\right)$ est un repère orthonormé composé de vecteurs propres. On a:

$$
g_{\alpha \beta}=e_{(0) x} e_{(0) \beta}-e_{(1) x} e_{(1) \beta}-e_{(2) \alpha} e_{(2) \beta}-e_{(3) \alpha} e_{(3) \beta}
$$

et

$$
\tau_{\alpha \beta}=k\left[e_{(0) \alpha} e_{(0) \beta}+e_{(1) \alpha} e_{(1) \beta}+e_{(2) z} e_{(2) \beta}-e_{(3) \alpha} e_{(3) \beta}\right] .
$$

Rapporté au repère $\left.\overrightarrow{(e}_{\alpha}\right)$, le tensenr de Maxwell donne un vecteur de Poynting nul et est nécessairement représenté par une matrice de la forme (5.2), avec $\xi_{\eta}=0$, puisque, d'après une remarque précédente, $\left(\overrightarrow{e_{\alpha}}\right)$ est un repère simple, Comme $\overrightarrow{e_{0}}$ et $\overrightarrow{e_{3}}$ ont même valeur propre, on a $\xi=0$, soit $\mathrm{Y}=M=0$.

Seuls $Z$ et $N$ ne sont pas nuls en général. Par suite, dans le repère précédent, $F$ et $* F$ admettent les expressions :

$$
\left\{\begin{array}{l}
F=-Z \theta^{0} \wedge \theta^{3}+N \theta^{1} \wedge \theta^{2} \\
* F=N \theta^{0} \wedge \theta^{3}+Z \theta^{1} \wedge \theta^{2}
\end{array}\right.
$$

$F$ et $* F$ sont linéairement indépendantes et il est clair qu'il n'existe aucune forme linéaire non nulle telle que les produits extérieurs de cette forme par $F$ et $* F$ scient tous deux nuls.

Nous observerons que $\tau_{\alpha \beta}$ admet deux vecteurs propres isotropes que l'on peut définir par:

$$
\vec{l}=\overrightarrow{e_{0}}+\overrightarrow{e_{3}} \quad \overrightarrow{l^{*}}=\overrightarrow{e_{0}}-\overrightarrow{e_{3}}
$$

Ces vecteurs sont aussi vecteurs propres de $F$ et $* F$ : de $(6 \cdot 3)$ on déduit par exemple :

$$
l^{x} F_{\alpha \beta}=-Z l_{\beta} \quad l^{\alpha}(* F)_{\alpha \beta}=N l_{\beta} .
$$


A. Licinerowicz: Ondes et radiations électromagnétiques, etc.

Inversement tout vecteur propre de $F$ est manifestement vecteur propre de $\tau_{\alpha \beta}$; par suite $s^{\prime}$ il est isotrope, il est colinéaire à $\vec{l}$.ou $\overrightarrow{l^{*}}$

\section{Le cas singulier.}

Pour $k=0$, on a $\Psi=\Phi=0$. Relativement à tout repère orthonormé, le champ électrique et le champ magnétique sont orthogonaux et ont même longueur:

a) Dans ce cas $\eta^{2}=\xi^{2}$ et par suite $\eta= \pm \xi$. Dans un repère simple, $\tau_{01}= \pm \xi^{2}$. En changeant $\overrightarrow{e_{1}}$ en $-\overrightarrow{e_{1}}$ et simultanément $\overrightarrow{e_{2}}$ en $-\overrightarrow{e_{2}}$ pour ne pas modifier l'orientation $d u$ repère, on peut faire en sorte que $\eta=-\xi$. Pour un tel repère, le tensenr Maxwell admet ponr composantes

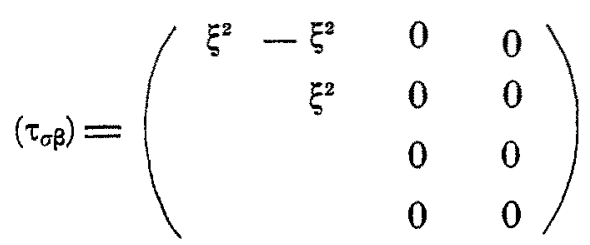

Introduisons le vecteur de longueur nulle:

$$
\vec{l}=\overrightarrow{e_{0}}+\overrightarrow{e_{1}}
$$

qui admet pour composantes covariantes:

$$
l_{0}=1 \quad l_{1}=-1 \quad l_{2}=l_{3}=0
$$

(7-1) peut ètre traduite par la relation.

$$
\tau_{\alpha \beta}=\xi^{2} l_{\alpha} l_{\beta}
$$

$\tau_{x \beta}$ admet pour vecteurs propres les vecteurs $d u$ 3-plan tangent le long de $\vec{l}$ au còne elémentaire $d s^{2}=0$. Ils définissent, sauf la direction isotrope définie par $\vec{l}$, des directions orientées dans l'espace. Il est done impossible ici de trouver un vecteur propre orienté dans le temps et par suite d'annuler le vecteur de Poynting. La forme (7.2) da tenseur d'impulsion énergie du champ électromagnétique, où $\vec{l}$ est un vecteur isotrope, est à rapprocher de la forme (4-7) du tenseur d'impulsion-énergie d'un fluide matériel sans pression.

b) Soit $\left(\overrightarrow{e_{x}}\right)$ un repère adapté. Si dans le 2-plan $\left(\vec{e}_{2}, \overrightarrow{e_{3}}\right), \vec{E}$ a pour composantes $(Y, Z)$, le vecteur $\vec{H}$ orthogonal à $\vec{E}$ et de même longueur a pour composantes $M=-\varepsilon Z$ et $N=\varepsilon Y$ (où $\varepsilon= \pm 1$ ). Comme $Y Z+M N=0$ le repère est simple. Ainsi, dans le cas singulier, tout repère adapté est simple. D' après (5.1), on a en repère simple:

$$
Z N-Y N=-\xi^{2} .
$$


Il vient:

$$
-\varepsilon\left(Y^{2}+Z^{2}\right)=-\xi^{2}
$$

et par suité $\varepsilon=1$. Ainsi, avec nos conventions de signe, on a en repère adapté :

$$
M=-\mathrm{Z} \quad N=Y .
$$

En utilisant ces expressions des composantes de $F$ et $* F$, il -vient:

$$
\begin{gathered}
F=Y \theta^{2} \wedge\left(\theta^{0}-\theta^{1}+Z \theta^{3} \wedge\left(\theta^{0}-\theta^{1}\right)\right. \\
* F^{\top}=Z \theta^{2} \wedge\left(\theta^{0}-\theta^{1}\right)-Y \theta^{3} \wedge\left(\theta^{0}-\theta^{1}\right) .
\end{gathered}
$$

En introduisant la forme linéaire $\lambda$ définie par $l_{\alpha}$ :

on obtient

$$
\lambda=l_{\alpha} \theta^{x}=\theta^{0}-\theta^{1}
$$

$$
\left\{\begin{array}{r}
F=\left(Y \theta^{2}+Z \theta^{3}\right) \wedge \lambda \\
* F=\left(Z \theta^{2}-Y \theta^{3}\right) \wedge \lambda
\end{array} .\right.
$$

On pourrait en particulier choisir, dans le 2 -plan $\left(\overrightarrow{e_{2}}, \overrightarrow{e_{3}}\right), \overrightarrow{e_{2}}$ parallèle au champ électrique et $\overrightarrow{e_{3}}$ parallèle au champ magnétique; on aurait alors $Z=0$.

Il est clair que pour qu' une forme linéaire annule à la fois $F$ et $* F$ il faut et il suffit qu' elle soit proportionelle à $\lambda$. L'existence de telles formes annulant simultanément $F$ et $* F$ caractérise le cas singulier. Notons que $\lambda \wedge(* F)=0$ est équivalent à $l^{\alpha} F_{\alpha \beta}=0$. Ainsi.

$$
l^{\alpha} F_{\alpha \beta}=0 \quad l^{\alpha}(* F)_{\alpha \beta}=0
$$

c) Soit $V_{m+1}$ une varieté de dimension $(m+1)$ munie d'uue métrique riemannienne de type hyperbolique normal. Si $F \neq 0$ est une 2-forme de cette variété, nous dirons que $F$ est une 2-forme singulière s'il existe un vectenr $\vec{l}$ tel que

$$
l_{\alpha} F_{\beta \gamma}+l_{\beta} F_{\gamma \alpha}+l_{\gamma} F_{\alpha \beta}=0 \quad(\alpha, \beta, \gamma=0,1, \ldots, m)
$$

et

$$
l^{\alpha} F_{\alpha \beta}=0 .
$$

Le vecteur $\vec{l}$ défini à un facteur scalaire près sera dit vecteur fondamental de $F$. Dans le cas de la relativité générale $(m=3),(7.5)$ et $(7.6)$ caractérisent bien les 2-formes correspondant an cas singulier puisque ces relations expriment qu'il existe une forme linéaire anuulant simultanément $F$ et $* F$ (qui est ici une 2-forme).

Dans le cas général le vecteur $\vec{l}$ est nécessairement isotrope. En effet s'il ne l'était pas on pourrait choisir un repère orthonormé $\left(\overrightarrow{e_{x}}\right)$ tel que l'un des vecteurs du repère, soit $\overrightarrow{e_{0}}$, soit colinéaire à $\vec{l}$. Désignons provisoirement 
par $u, v$ des indices prenant les valeurs $1, \ldots, m$. En faisant $\alpha=0, \beta=u$, $\gamma=v$ dans (7.5), il vient $F_{u n}=0$. D'autre part d'après. $(7-6), F_{\alpha \beta}=0$. Ainsi si $\vec{l}$ n'était pas isotrope, nécessairement $F=0$.

Il est aisé de dédnire de (7-5), (7-6) des expressions de $F$ qui nous seront commodes pour la suite.

Au point $x$ de $V_{m+1}$, donnous-nous une direction isotrope définie par nn vecteur $\vec{l}$ et désignons par $\left.\overrightarrow{(n}^{(t)}\right)(i=1, \ldots, m-1)$ un système de $(m-1)$ vectenrs orthogonaux, normés, tangents au cône élémentaire le long de la génératrice définie par $\vec{l}$. Au $(m-1)$-plan déterminé par les $\vec{n}^{(i)}$ est orthogonal un 2-plan orienté dans le temps et contenant $\vec{l}$. Dans ce 2-plan choisissons un vecteur unitaire $\left.\overrightarrow{e_{0}} \overrightarrow{\left(e_{n}^{2}\right.}=1\right)$ arbitraire et soit $\overrightarrow{e_{m}}$ le vecteur normé orthogonal $\dot{a} \vec{l}_{0}^{*}$ tel que nous puissions prendre $\overrightarrow{e_{0}}+\overrightarrow{e_{m}}=\vec{l}$. Considérons le repère ortho. norme $\overrightarrow{\left(\vec{e}_{k}\right)}$ défini par $\vec{e}_{0}, \vec{e}_{i}=\vec{n}^{(i)}, \vec{e}_{m} ;$ si $F$ est une 2-forme singulière de vecteur fondamental isotrope $\vec{l},(7-5)$ se traduit par:

$$
F_{i j}=0 \quad F_{0 t}+F_{m i}=0
$$

et (7.6) par:

$$
F_{\beta \beta}+F_{m \beta}=0
$$

et la forme $F$ est déterminée par la connaissance des $(m-1)$ nombres $a_{i}=F_{i 0}$.

Introduison les $(m-1)$ 2-forme singulières $\varphi^{(t)}$ définies par:

$$
\varphi_{\alpha \beta}^{(i)}=l_{\alpha} n_{\beta}^{(i)}-l_{\beta} n_{\alpha}^{(i)} .
$$

Les carrés et produits scalaires de ces formes sont manifestement nuls. Dans le repère $\overrightarrow{\left(e_{\lambda}\right)}$ envisagé :

$$
\varphi_{j 0}^{(i)}=-n_{j}^{(i)}=\delta_{j^{*}}^{i}
$$

Il en résulte que si $F$ est une 2-forme singulière de vecteur fondamental $\vec{l}$ :

$$
F_{\alpha \beta}=\sum_{i} a_{i} \varphi_{\alpha \beta}^{(i)}=\sum_{i} a_{i}\left(l_{\alpha} n_{\beta}^{(i)}-l_{\beta} n_{\alpha}^{(i)}\right)
$$

$F$ et $\vec{l}$ étant donnés, les $a_{i}$ ne peurent dépendre que du système des $\vec{n}^{(i)}$. Ce système peut soit subir la transformation $\overrightarrow{n^{(t)}} \rightarrow \overrightarrow{n^{(t)}}+\overrightarrow{k^{(t)}} \vec{i}$, soit subir une rotation dans le $(m-1)$-plan qu'il détermine. Dans le premier cas, les formes $\varphi^{(t)}$ et par suite les $a_{i}$ ne sont pas modifiés. Dans le second cas on peut laisser fixes $\overrightarrow{e_{0}}, \overrightarrow{e_{m}}$ et les $F_{i 0}$ déterminent dans le $(m-1)$-plan envisagé un vecteur; les $a_{i}$ sont donc les composantes d'un vecteur du $(m-1)$-plan $\left(\overrightarrow{\left.n^{(i)}\right)}\right.$.

Si l'on pose $b_{\alpha}=\Sigma a_{i} n_{x}^{(i)}$, on voit $q u^{\prime}$ il existe un vecteur $b_{\alpha}$ orthogonal à $l_{x}$ tel que :

$$
F_{\alpha \beta}=l_{\alpha} b_{\beta}-l_{\beta} b_{\alpha}
$$


Un tel vecteur est défini à la transformation près $b_{\alpha} \rightarrow b_{\alpha}+k l_{\alpha}$. On notera que le scalaire positif :

$$
\left|b^{2}\right|=-b^{x} b_{x}=\Sigma^{q}\left(a_{i}\right)^{2}
$$

ne dépend que la forme $F$ et du choix du vecteur $\vec{l}$.

d) Etablissons enfin le lemme suivant pour une 2-forme $F$ quelcon. que de $V_{4}$ :

LEMme. - Pour qu'un vecteur propre $\vec{l}$ de la forme $F \neq 0$ soit aussi vecteur propre de $* F$, il faut et il suffit qu'il soit isotrope.

En effet si $l$ est vecteur propre commun de $F$ et $* F$ :

On en déduit:

$$
l^{\star} F_{\alpha \beta}=a l_{\beta} . \quad l^{\alpha}(* F)_{\alpha \beta}=b l_{\beta} .
$$

$$
a l_{\beta} l^{\beta}=0 \quad b l_{\beta} l^{\beta}=0 .
$$

Si $\vec{l} n^{\prime}$ etait pas isotrope, on aurait $a=b=0$ et, les relatious (7-5), (7-6) etant satisfaites, on en deduirait $F=0$. Inversement si $l^{\alpha}$ ezt un vecteur propre isotrope de $F$, il est vecteur propre isotrope de $\tau_{\alpha \beta}$ donc de $(* F)$, que $F$ soit régulière ou singulière.

\section{Discontinuites des dérivées du champ éleciromagnétique.}

a) En l'absence de tout phénomène d'induction, considérons un champ électromagnétique qui, dans le domaine envisagé, satisfait aux équations de Maxweul avec un vecteur courant électrique continu. Le champ $F_{\alpha \beta}$ étant par hypothèse de classe $\left(C^{0}, C^{2}\right.$ par morceaux), nous somme conduits à étudier à la traversée de quelles hypersurfaces $S$ les dérivées premières du champ présentent des discontinuités ainsi que la structure mème de ces discontinuites.

Nous désignerons par $f\left(x^{\alpha}\right)=0$ l'équation locale d'une hypersurface $S$ à la traversée de laquelle se produisent de telles discontinuités. Le tenseur métrique étant de classe $\left(C^{1}, C^{3}\right.$ par morceaux), la connexion riemannienne associée est continue et si l'on note avec le signe [ ] la discontinuité d'une quantité à la traversée de $S$ on a

$$
\left[\nabla_{\gamma} F_{\alpha \beta}\right]=\left[\theta_{\gamma} F_{\alpha \beta}\right] \quad\left(\partial_{\gamma}\right. \text { dérivée pfaffienne). }
$$

On déduit immédiatement des conditions de HADAMARD sur la propagation des ondes qu'il existe, aux points de $S$, un tenseur antisymétrique $\varphi_{x B}$ tal que:

$$
\left[\nabla_{\gamma} F_{\alpha \beta}\right]=\varphi_{\alpha \beta} l_{\gamma}
$$

où $l_{\gamma}=\partial_{\gamma} f$. Désignons par $\varphi$ la 2-forme définie aux points de $S$ par le tenseur $\varphi_{\alpha \beta}$. En antisymétrisant (8-1), il résulte de $[d F] \doteq 0$ :

$$
l_{\alpha} \varphi_{\beta \gamma}+l_{\beta} \varphi_{\gamma \alpha}+l_{\gamma} \varphi_{\alpha \beta}=0 \text {. }
$$


En contractant (8-1), il résulte de $[\delta F]=0$ :

$$
l^{\alpha \varphi_{x p}}=0 \text {. }
$$

Ainsi la forme $\varphi$ est singulière en tout point de $S$ et $\vec{l}$ est nécessairement isotrope. L'hypersurface $S$ satisfait à l'équation aux dérivées partielles du premier ordre:

$$
\triangle_{1} f=g^{\alpha \beta} \partial_{\alpha} f \partial_{\beta}=0 .
$$

Les fronts d' onde électromagnétique, ou varietés caractéristiques des équations de MAXWELL, sont les hypersurfaces tangentes en chacan de leurs points an còne élémentaire de ce point. D'autre part $l_{\alpha}=a_{x} f$ étant un gradient:

$$
\nabla_{\beta} l_{\alpha}-\nabla_{\alpha} l_{\beta}=0 \text {. }
$$

Par suite

$$
l \beta\left(\nabla_{\beta} l_{\alpha}-\nabla_{\alpha} l_{\beta}\right)=0
$$

et $\vec{l}$ étant de longueur nulle, on a :

$$
l^{\beta} \nabla_{\beta} l_{x}=0
$$

qui exprime que les trajectoires sur $S$ du champ de vecteurs $\vec{l}$ sont des géodésiques de longueur nulle. Ainsi les rayons électromagnétiques on bicaractéristiques, qui sont les caractéristiques de (8.4), sont des géodésiques de longueur nulle de la métrique.

b) Soit $\left(\vec{e}_{x}\right)$ un repère en $x \in S$ adapté à la forme $\varphi$. Si

$$
\lambda=\theta^{\circ}-\theta^{2} \text {. }
$$

Il existe un scalaire $a$ tel que $d f=a \lambda$. D'après (7.3), on peat trouver deux nombres $Y$ et $Z$ tels que $\varphi$ s'exprime par:

$$
\varphi=\frac{1}{a}\left(Y \theta^{2}+Z \theta^{3}\right) \wedge \lambda
$$

De (8.1) il résulte que, relativement au repère envisagé, les seules compo. santes non nulles du tenseur $\left[\nabla_{Y} F_{\alpha \beta}\right]$ sont données par:

$$
\left[\nabla_{0} F_{20}\right]=-\left[\nabla_{0} F_{21}\right]=-\left[\nabla_{1} F_{20}\right]=\left[\nabla_{1} F_{21}\right]=Y
$$

et

$$
\left[\nabla_{0} F_{30}\right]=-\left[\nabla_{0} F_{3 !}\right]=-\left[\nabla_{1} F_{30}\right]=\left[\nabla_{1} F_{31}\right]=Z .
$$

On peut d'ailleurs choisir le repère $\left(\vec{e}_{x}\right)$ de façon que $Z=0$, les seules discontinuités non nulles étant alor's donnés par (8-6). Nous avons ainsi pu ramener à une forme canonique les composantes du tenseur $\left[\nabla_{\gamma} F_{x \beta}\right]$.

c) On peut observer qu'il est possible de choisir au voisinage d'un 
point $x_{0}$ de $S$ un système de coordonnées locales par rapport auquel $S$ admette une équation simple et qui soit tel que son repère naturel en $x_{0}$ coincide avec un repère adapté donné $\left(\vec{e}_{\alpha}\right)$, En effet soit $\theta_{0}^{\alpha}$ les formes linéaires en $x_{0}$ telles que:

$$
\underset{0}{\theta x}\left(\overrightarrow{e_{\beta}}\right)=\delta_{\beta}^{\alpha}
$$

et soit $u$ une variable telle que l'équation locale de $S$ soit $u=0$; de l'étude antérieure il résulte qu'il existe un nombre $a$ tel que:

$$
(d u)_{0}=a\left(\theta^{0}-\theta^{1}\right)_{0} .
$$

En modifiant $a$ d' un facteur constant, on peut faire $a=1$. Cela posé, étant donné une forme linéaire en $x_{0}$, il existe toujours au voisinage de $x_{0}$ une fonction locale de classe $\left(C^{2}, C^{4}\right)$ par morceaux) telle que sa différentielle coïncide en $x_{0}$ avec la forme linéaire. Nous pouvons ainsi trouver trois telles fonctions $v, x^{2}, x^{3}$ telles que en $x_{0}$

$$
(d v)_{0}=\left(\theta^{0}+\theta^{1}\right)_{0} \quad\left(d x^{2}\right)_{0}=\theta_{0}^{2} \quad\left(d x^{2}\right)_{0}=\theta_{0}^{3} .
$$

Si nous posons:

nous royons que:

$$
2 x^{0}=v+u \quad 2 x^{1}=v-u
$$

$$
\left(d x^{\alpha}\right)_{0}=\theta_{0}^{\alpha}
$$

D'après l'indipendance des formes linéaires $\theta_{0}^{x}$, les 4 fonctions $x^{\alpha}$ sont à jacobien non nul en $x_{0}$. Nous avons ainsi defini des coordonnées locales $\left(x^{\alpha}\right)$ telles que $S$ admette l'équation $x^{0}-x^{1}=0$ et pour lesquelles le repère naturel en $x_{0}$ est le repère adapté donné. Dans ces coordonnés

$$
\left[\nabla_{\alpha} F_{\alpha \beta}\right]=-\left[\nabla_{2} F_{\alpha \beta}\right]=\varphi_{\alpha \beta} \quad\left[\nabla_{2} F_{\alpha \beta}\right]=\left[\nabla_{3} F_{\alpha \beta}\right]=0 .
$$

\section{Relation différentielle portant sur $\left[\nabla_{\alpha} F_{\beta \gamma}\right]$.}

Supposons qu'à la traversée de $S$, le tenseur de courbure de $V_{4}$ soit continu. Le tenseur $\left[\nabla_{\alpha} F_{\beta \gamma}\right]$ satisfait alors sur $S$ à une relation différentielle remarquable que nous allons former, pouvu que $J^{\alpha}$ soit nul.

Soit $f\left(x^{x}\right)=0$ l'équation locale de $S$, où $f$ est supposée de classe $C^{2}$; le veoteur $l_{\alpha}=\partial_{\alpha} f$ est par suite de classe $C^{1}$ et $\nabla_{\beta} l_{\alpha}$ est continu à la traversée de $S$. De plus $l_{\alpha}$ étant un gradient:

$$
\nabla_{\alpha} l_{\beta}-\nabla_{\beta} l_{\alpha}=0 \text {. }
$$

a) Sur un voisinage $U$, adoptons des coordonnées locales telles que $S$ admette l'équation $x^{0}=0$. On a alors $g^{00}=0$ et le vecteur $l_{\alpha}$, gradient de $x^{0}$, admet les composantes covariantes :

$$
l_{0}=1 \quad l_{u}=0 \quad(u=1,2,3)
$$


Ses composantes contravariantes sont done:

$l_{*}$ étant de longueur nulle

$$
l^{0}=0 \quad l^{u}
$$

En particulier

$$
l_{x} \nabla_{\beta} l^{\alpha}=l_{0} \nabla_{\beta} l^{0}+l_{u} \nabla_{\beta} l^{u}=\nabla_{\beta} l^{0}=0 .
$$

Le tenseur $\left[\nabla_{\sigma} F_{\beta \gamma}\right]$ satisfait sur $S$ aux relations :

$$
l_{x}\left[\nabla_{\sigma} F_{\beta \gamma}\right]+l_{\beta}\left[\nabla_{\sigma} F_{\gamma x}\right]+l_{\gamma}\left[\nabla_{\sigma} F_{x \beta}\right]=0
$$

et

$$
l_{\alpha}\left[\nabla_{\sigma} F^{\alpha_{\beta}}\right]=0 .
$$

Dans les coordonnées locales adoptées, (9.3) s'écrit :

$$
\left[\nabla_{\sigma} F_{u v}\right]=0
$$

et $(9.4)$ :

$$
\left[\nabla_{\sigma} F_{\beta}^{0}\right]=0 .
$$

On notera enfin que $\nabla_{u}[]=\left[\nabla_{u}\right]$ et que, $F$ et le tenseur de courbure étant continus à la traversée de $S$, il résulte de l'identité de Ricor que:

$$
\left[\nabla_{\rho} \nabla_{\sigma} F_{\beta \gamma}\right]=\left[\nabla_{\sigma} \nabla_{\rho} F_{\beta \gamma}\right]
$$

b) La relatiou (9-3) étant satisfaite sur $S$, on peut la dériver sur cette hypersurface et il vient:

$$
\nabla_{u}\left(l\left[\nabla_{\sigma}\left[F_{\beta \gamma}\right]\right)+\nabla_{u}\left(l_{\beta}^{\beta}\left(\nabla_{\sigma} F_{\Upsilon}^{\alpha}\right]\right)+\nabla_{u_{u}}\left(l_{\Upsilon}\left[\nabla_{\sigma} F_{\beta}^{\alpha}\right]\right)=0\right.
$$

soit en donnant à $\alpha$ la valeur $u$ et sommant:

$$
l^{u} \nabla_{u}\left[\nabla_{\sigma} F_{\alpha \beta}\right]+\left(\nabla_{u} l^{u}\right)\left[\nabla_{\sigma} F_{\beta \gamma}\right]+Q_{\sigma, \beta_{\gamma}}=0
$$

où l'on a posé:

$$
Q_{\sigma, \beta Y}=\nabla_{u} l_{\beta}\left[\nabla_{\sigma} F_{Y}{ }^{u}\right]+\nabla_{u} l_{Y}\left[\nabla_{\sigma} F^{u} \beta\right]+l_{\beta}\left[\nabla_{u} \nabla_{\sigma} F_{Y}{ }^{u}\right]+l_{Y}\left[\nabla_{u} \nabla_{\sigma} F^{u_{\beta}}\right]
$$

En tenant compte de (9-2) et (9.6), on a :

$$
l P \nabla_{p}\left[\nabla_{\sigma} F_{\beta \gamma}\right]+\left(\nabla_{\rho} l l\right)\left[\nabla_{\sigma} F_{\beta_{\gamma}}\right]+Q_{\sigma, \beta \gamma}=0
$$

avec

$$
Q_{\sigma, \beta_{Y}}=\left[\nabla_{\beta} l_{\rho} \nabla_{\sigma} F_{Y}^{{ }^{p}}+\nabla_{Y} l_{\rho} \nabla_{\sigma} F \rho_{\beta}\right]+l_{\beta}\left[\nabla_{\sigma} \nabla_{u} F_{Y}{ }^{\mu}\right]+l_{Y}\left[\nabla_{\sigma} \nabla_{u} F^{u}{ }_{\beta}\right]
$$


De la nullité du vecteur-courant il résulte:

$$
Q_{\sigma, \beta \gamma}=\left[\nabla_{\beta} l_{\rho} \nabla_{\sigma} F_{Y}{ }^{\rho}+\nabla_{Y} l_{\rho} \nabla_{\sigma} F \rho_{\beta}\right]-l_{\beta}\left[\nabla_{\sigma} \nabla_{0} F_{Y}^{0}\right]-l_{\gamma}\left[\nabla_{\sigma} \nabla_{0} F^{\prime}{ }_{\beta}\right]
$$

Or:

$$
\nabla_{\beta} l_{\rho} \nabla_{\sigma} F_{Y} \rho+\nabla_{Y} l_{\rho} \nabla_{\sigma} F \rho_{\beta}=\nabla_{\beta}\left(l_{\rho} \nabla_{\sigma} F_{Y}{ }^{\rho}\right)+\nabla_{Y}\left(l_{\rho} \nabla_{\sigma} F \rho_{\beta}\right)-l_{\rho}\left(\nabla_{\beta} \nabla_{\sigma} F_{Y} \rho+\nabla_{Y} \nabla_{\sigma} F_{\rho} \rho_{\beta}\right)
$$

où la discontinuitè relative au dernier terme peut être évaluée en tenant compte de $d F=0$. On a ainsi :

$$
Q_{a} ; \beta_{Y}=l \rho \nabla_{\rho}\left[\nabla_{\sigma} F_{\beta Y}\right]+\left[\nabla_{\beta}\left(l_{\rho} \nabla^{\sigma} F_{Y}{ }^{\rho}\right)+\nabla_{Y}\left(l_{\rho} \nabla_{\sigma} F_{\beta} \beta\right)\right]-l_{\beta}\left[\nabla_{0} \nabla_{\sigma} F_{Y}^{0}\right]-l_{Y}\left[\nabla_{0} \nabla_{\sigma} F_{\beta}^{0}\right] .
$$

Or pour $\beta=u$

$$
\left[\nabla_{u}\left(l_{P} \nabla^{\sigma} F_{Y}{ }^{\rho}\right)\right]=\nabla_{u}\left(l_{\rho}\left[\nabla_{\sigma} F_{Y^{\rho}}\right]\right)=0
$$

Il en résulte :

$$
Q_{\sigma, u v}=l P \nabla_{\rho}\left[\nabla_{\sigma} F_{u v}\right] .
$$

D'autre part pour $\beta=0, \gamma=u$

$$
\left[\nabla_{0}\left(l_{\rho} \nabla^{\sigma} F_{u^{p}}\right)\right]=\left[\nabla_{0} l \rho \nabla_{\sigma} F_{u_{\rho}}+l_{\rho} \nabla_{0} \nabla_{\sigma} F_{u^{p}}\right]=\left[\nabla_{0} \nabla_{\sigma} F_{u^{0}}\right]
$$

puisque $\nabla_{0} l^{0}=0$. On a ainsi :

Soit d'après $(9 \cdot 10)$

$$
Q_{\sigma, 0 u}=l \rho \nabla_{\rho}\left[\nabla_{\sigma} F_{0 u}\right]
$$

$$
Q_{\sigma, B_{Y}}=l_{\rho} \nabla_{\rho}\left[\nabla_{\sigma} F_{\beta Y}\right] .
$$

En reportant dans (9-8), on voit que le tenseur $\left[\nabla_{\gamma} F_{\alpha \beta}\right]$ satisfait sur $S$ a la relation différentielle:

$$
2 l \rho \nabla_{\rho}\left[\nabla_{\gamma} F_{\alpha \beta}\right]+\left(\nabla_{\rho} l \rho\right)\left[\nabla_{r} F_{\alpha \beta}\right]=0
$$

qui met en évidence la propagation des discontinuites de $\nabla_{Y} F_{\alpha \beta}$ le long des géodésiques de longueur nulle de $S$. En particulier si, en un point $x$ de $S$, $\left[\nabla_{\gamma} F_{\alpha \beta}\right]=0$ il en est de même tout le long de la géodésique de longueur nulle issue de $x$ et située sur $S$.

En posant dans (9-11) $\left[\nabla_{\gamma} F_{\alpha \beta}\right]=\varphi_{\alpha \beta} l_{\gamma}$, il vient, compte-tenu de $l_{\rho} \nabla_{\rho} l_{\gamma}=0$

$$
2 l \rho \nabla_{\rho} \varphi_{\alpha \beta}+\left(\nabla_{\rho} l \rho\right) \varphi_{\alpha \beta}=0 .
$$

Soit $\Sigma$ une hypersurface orienté dans l' espace et coupant $S\left(x^{0}=0\right)$ selon une 2-surface $U$. Donnons nous aux points de $\Sigma$ une 2-forme $\left(F_{\alpha \beta}\right)_{\Sigma}$ dont les dérivées premières présentent à la traversée de $S$ des discontinuités $\left[\partial_{0} F_{\alpha \beta}\right]_{U}=$ $=\left(\varphi_{\alpha \beta}\right)_{U}$, où $\left(\varphi_{\alpha \beta}\right)_{U}$ est une 2-forme définie aux points de $U$, singulière de vecteur fondamental $\left(l_{\alpha}\right)_{U}$, donnée arbitrairement. A partir de la donnée de CAUCHY $\left(F_{x \beta}\right)_{\Sigma}$ sur $\Sigma$, les équations de MaXweLL du vide déterminent en dehors de $\Sigma$ un champ électromagnétique dont les dérivées premières sont 
A. LuChinerowicz: Ondes et radiations électromagnétiques, etc.

discontinues à la traversée de $S$. Le tenseur de discontinuité $\varphi_{x \beta}$ correspondant est nécessairement la solution manifestement singulière de (9.12) correspondant à la donnée initiale $\left(\varphi_{x \beta}\right)_{U}$ sur $U$.

\section{Une identité de conservation.}

$\varphi_{\alpha \beta}$ étant nne 2-forme singulière de vecteur fondamental $l_{\beta}$, il existe un vecteur $b_{\rho}$ orthogonal à $l_{\rho}$ tel que

$$
\varphi_{\alpha \beta}=l_{\alpha} b_{\beta}-l^{\beta} b_{\alpha} .
$$

En reportant dans (9.12) cette expression de $\varphi_{a \beta}$, il vient, compte-tenu de $l r \nabla_{\rho} l_{x}=0$

$$
l_{\alpha}\left\{2 l^{\circ} \nabla_{\rho} b_{\beta}+\left(\nabla_{\rho} l^{o}\right) b_{\beta}\right\}-l_{\beta}\left\{2 l^{b} \nabla_{\rho} b_{\alpha}+\left(\nabla_{\rho} l_{p}\right) b_{\alpha}\right\}=0
$$

soit :

$$
2 l p \nabla_{p} b_{x}+\left(\nabla_{p} l p\right) b_{\alpha}=k l_{x}
$$

où $k$ est un scalaire. En multipliant les deux membres de cette relation par $b^{x}$ on obtient:

$$
2 l \rho b^{x} \nabla_{\rho} b_{\alpha}+\left(\nabla_{\rho} l \rho\right) b^{x} b_{\alpha}=0
$$

soit

$$
l_{p} \nabla_{p}\left|b^{2}\right|+\left(\nabla_{p} l^{p}\right)\left|b^{2}\right|=0
$$

on en déduit l'identité

$$
\nabla_{p}\left(\left|b^{2}\right| l p\right)=0
$$

$F$ étant donnée, le vecteur le est défini à un facteur $\lambda$ près constant le long des géodésiques isotropes trajectoires de $l \rho$. Si $l p \rightarrow \lambda l_{p}, \varphi_{\alpha \beta} \rightarrow \lambda^{-1} \varphi_{\alpha \beta}$ et par suite $b_{x} \rightarrow \lambda^{-2} b_{x}$. Ainsi $\left|b^{2}\right| \rightarrow \lambda^{-4}\left|b^{2}\right|$. Le tenseur

$$
\tau_{\alpha \beta_{Y} \dot{\delta}}=\left|b^{2}\right| l_{x} l_{\beta} l_{Y} l_{\delta}=-\left[\nabla_{\alpha} F \rho_{\gamma}\right]\left[\nabla_{\beta} F_{\rho \delta}\right]
$$

ne dépend que de $F_{a p}$ et d'après (10-1) est conservatif:

$$
\nabla_{\alpha} \tau_{\beta \gamma \delta}^{\alpha}=0
$$

\section{Radiation électromagnétique pure.}

a) L'étude faite au $\$ 8$ nous a montré que la présence de discontinnités des dérivées du champ électromagnétique à la traversée d'une hypersurface $S$ définit aux points de $S$ une 2-forme singulière. Nous sommes ainsi conduits à représenter une radiation électromagnétique pure dans le vide par un champ $F$ défini par une forme singulière. Pour un tel champ, il existe d'après (7.2) un champ de vecteurs isotropes $\vec{m}$ tel que le tenseur de MaxwELL puisse s'écrire :

$$
\tau_{x \beta}=m_{x} m_{\beta}
$$


Exprimons à partir de (11.1) des conditions de conservation :

$$
\nabla_{\alpha} \tau^{\alpha \beta}=0 \text {. }
$$

Il vient :

$$
\left(\nabla_{\alpha} m^{\alpha}\right) m^{\beta}+m^{\alpha} \nabla_{\alpha} m^{\beta}=0
$$

soit

$$
m^{x} \nabla_{a} m^{\beta}=-\left(\nabla_{a} m x\right) m^{\beta} .
$$

L'équation (11-3) exprime que la différentielle absolue du vecteur $\vec{m}$ dans sa propre direction est colinéaire à $\vec{m}$. Il en résulte que les trajectoires du champ de vecteurs $\vec{m}$ sont autoparallèles, c'est-è-dire sont des géodésiques de longueur nulle de la métrique.

L'étude des géodésiques d' une connexion linéaire montre qu'il existe, le long de chaque géodésique, un "paramètre affine» $\sigma$, défini à une transformation $\sigma \rightarrow a \sigma+b$ près, tel que, pour le vecteur-vitesse $\vec{l}=d x / d \sigma$ correspondant, on ait

$$
l^{\infty} \nabla_{\alpha} l^{\beta}=0 .
$$

Le vecteur $d x / d \sigma$ est ainsi défini à un facteur scalaire près, constant le long de chaque géodésique.

A $\vec{m}$ nous sommes ainsi conduits à substituer $\vec{l}$ qui lui est colinéaire et satisfait (11.4). Posons

$$
m_{r}=\pi l_{\alpha}
$$

où $\pi$ est un scalaire. Il vient ainsi :

$$
\tau_{\alpha \beta}=\pi^{2} l_{\alpha} l_{\beta}
$$

et les conditions de conservation s'ecrivent :

$$
\nabla_{\alpha}\left(\pi^{2} l^{\alpha}\right) l^{\beta}+\pi^{2} l_{\alpha} \nabla_{\alpha} l^{\beta}=0 .
$$

Il en résulte que, par l'introduction du champ de vecteurs $l^{\alpha}$ considéré, les conditions de conservation du tenseur de MAXwelu se traduisent par (11.4) et la relation

$$
\nabla_{\alpha}\left(\pi^{2} l^{\alpha}\right)=0
$$

Ainsi un champ électromagnétique singulier est un champ tel que son ten. seur de Maxwell paisse se mettre sous la forme (11.5) où $\vec{l}$ est un champ de vecteurs isotropes tel que $i(\overrightarrow{l)} \cdot F=\overrightarrow{i(l)}(* F)=0$ (où $i(\overrightarrow{l)}$ est l'opérateur "produit intérieur» par $\overrightarrow{l)}$ et qui peut être astreint à satisfaire (11-4). Ce champ peut-être assimilé à un fluide de photons dont les «lignes de courant» trajectoires de $\vec{l}$ sont des rayons électromagnétiques géodésiques de longueur nulle et qui admet une équation de continuité (11-6). Un tel champ traduit 
une radiation électromagnétique dont la propagation est bien régie par le còne élémentaire.

b) Si $\vec{l}$ definit un champ de vecteurs isotropes tel que (11.5) soit satisfaite, la dérivée de Lie de $\vec{F}$ pour le champ $\vec{l}$, soit $L \overrightarrow{(l)} F$, est donnée par:

$$
L \overrightarrow{(\vec{l})} F=d \overrightarrow{i(\vec{l})} F+\overrightarrow{i(\vec{l})} d F .
$$

Comme $i \overrightarrow{(l)} F=0, d F=0$, il vient:

$$
L(\vec{l}) F=0 .
$$

Ainsi $F$ est invariante par le champ de vecteurs $\vec{l}$. Si $F$ est nulle en un point $x$ de $V_{4}$, elle est nulle sur toute la géodésique isotrope trajectoire de $\vec{l}$ issue de $x$. Sous forme explicite (11-7) s'écrit

$$
l_{\rho} \nabla_{\rho} F_{\alpha \beta}-\nabla_{\alpha} l_{\rho} F_{\beta}{ }^{p}-\nabla_{\beta} l_{\rho} F_{\rho_{x}}=0 .
$$

De cette relation, on peut rapprocher une relation un peu différente que nous allons former. Par hypothèse:

$$
l \rho F_{\alpha \beta}+l_{\alpha} F_{\beta}^{\rho}+l_{\beta} F_{\rho}=0 .
$$

On en dédait par dérivation, compte-tenu des équations de Maxwell du vide:

$$
\nabla_{\rho}\left(l_{\rho} F_{\alpha \beta}\right)+\nabla_{\rho} l_{\alpha} F_{\beta} \rho+\nabla_{\rho} l_{\beta} F_{\alpha}=0 .
$$

c) $F$ étant singulière, on sait $q u^{\prime}$ il existe un vecteur $b_{\rho}$ orthogonal à $l_{\rho}$ tel que:

$$
F_{x \beta}=l_{\alpha} b_{\beta}-l_{\beta} b_{\alpha} .
$$

Le tenseur de MaxweLl $\tau_{x \beta}$ admet ainsi l'expression suivante:

soit :

$$
\tau_{\alpha \beta}=-F_{x}{ }^{2} F_{\beta \rho}=-\left(l_{\alpha} b_{\rho}-l \rho b_{\alpha}\right)\left(l_{\beta} b_{\rho}-l_{\rho} b_{\beta}\right)
$$

$$
\tau_{\alpha \beta}=-\left(b \cdot b_{\rho}\right) l_{\alpha} l_{\beta}=\left|b^{2}\right| l_{\alpha} l_{\beta} .
$$

Ainsi pour le mème choix du vecteur $l_{x}$ satisfaisant $(11-4), \pi^{1}=\left|b^{2}\right|$ et $(11-6)$ s'ecrit :

$$
\nabla_{p}\left(\left|b^{2}\right| l p\right)=0
$$

d) L'identité (11-11) peut ètre établie par une méthode directe ne faisant pas intervenir le tenseur de MAXwnLL et qui peut être étendue au cas gravitationnel. En substituant (11-10) dans (11-8) il vient:

$$
l_{x} l_{\rho} \nabla_{\rho} b_{\beta}-l_{\beta} l_{\rho} \nabla_{\rho} b_{x}-\nabla_{\alpha} l_{\rho} l_{\rho} b^{\rho}+\nabla_{\beta} l_{\rho} l_{x} b_{\rho}=0 .
$$

En multipliant par $b^{x}$ on obtient:

$$
l \rho b \times \nabla_{\rho} b_{\alpha}+\nabla_{\alpha} l_{\rho} b^{\alpha} b^{a}=0 .
$$


En precédant de même à partir de (11.9), on obtient d'abord:

$$
l_{\alpha} l_{\rho} \nabla_{\rho} b_{\beta}-l_{\beta} l_{\rho} \nabla_{\rho} b_{\alpha}+\left(\nabla_{\rho} l \rho\right)\left(l_{\alpha} b_{\beta}-l_{\beta} b_{\alpha}\right)+\nabla_{\rho} l_{\alpha} l_{\beta} b^{\rho}-\nabla_{\rho} l_{\beta} l_{\alpha} b_{\rho}=0 .
$$

.Aprés multiplication par $b_{\alpha}$, il vient:

$$
l \rho b^{\alpha} \nabla_{\rho} b_{\alpha}+\left(\nabla_{\rho} l \rho\right) b^{\alpha} b_{\alpha}-\nabla_{\rho} l_{\alpha} b^{\alpha} b_{\rho}=0 .
$$

Par addition membre à membre de (11-12) et (11-13) on obtient:

$$
2 l \rho b^{x} \nabla_{p} b_{\alpha}+\left(\nabla_{p} l \rho\right) b^{\alpha} b_{\alpha}=0
$$

c'est-à-dire l' identité (11-11).

\section{Champ électromagnétique de type intégrable.}

Sur un voisinage $U$ de $V_{4}$, donnons-nous un champ électromagnétique $F$ quelconque. En un point $x$ où $F$ est régulière, elle admet deux directions propres isotropes distinctes, en un point où elle est singulière une seule. Considérons le 3-plan $\Pi(x)$ tangent en $x$ au cône élémentaire $C_{2}$ le long d'une telle génératrice du cône. Nous pouvons ainsi associer à $F$. de deux façon au plus, un champ II de 3-plan tangents aux cônes élémentaires. Nous dirons que le champ électromagnétique est de type intégrable si le champ II est complètement intégrable. Pour qu'il en soit ainsi, il faut et il suffit que II admette une définition par une équation de la forme $d \sigma=0$, où $\sigma$ est une fonction, e'est-à-dire $q u^{\prime}$ on puisse définir le champ de directions isotropes envisagé par un champ de gradient.

Si $l_{\alpha}$ est un gradient, on a:

$$
\nabla_{\beta} l_{\alpha}-\nabla_{a} l_{\beta}=0
$$

et par suite

$$
l^{\beta}\left(\nabla_{\beta} l_{\infty}-\nabla_{a} l_{\beta}\right)=0
$$

c'est-à-dïre, $l_{\alpha}$ étant de longueur nulle,

$$
l^{\beta} \nabla_{\beta} l_{\alpha}=0 \text {. }
$$

Ainsi, pour un champ de type intégrable, les trajectoires de l'un des champs de directions isotropes sont encore des géodésiques de longueur nulle.

\section{Permanence du champ singulier de type intégrable.}

Considérons un champ électromagnétique $F$ de type intégrable satisfaisant aux équations de Maxwell du vide et supposons $q \mathrm{u}^{\prime}$ il existe une hypersurface $\Sigma$ orientée dans l'espace sur laquelle $F$ soit singulier.

Dire que $F$ est de type intégrable, c'est dire qu'il existe un champ de vecteurs isotropes $\vec{l}$ qui sont des gradients et qui satisfont $\grave{a}$ :

$$
l_{\alpha} F^{x \beta}=a l^{\beta} .
$$


On a alors aussi :

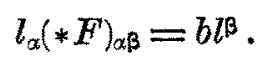

Sur $\Sigma$, la forme $F$ est singulière et par suite nécessairement $a=b=0$ sur $\Sigma$. En dérivant (13-1), il vient:

$$
\nabla_{\beta}\left(\alpha l^{\beta}\right)=\nabla_{\beta} l_{\alpha} F^{\alpha \beta}+l_{\alpha} \nabla_{\alpha} F^{\alpha \beta} \text {. }
$$

De (12-1) et des équations de MaXweli, il résulte que le second membre est nul. Ansi :

$$
\nabla_{\beta}\left(a l^{\beta}\right)=l^{\beta} \gtrsim_{\beta} a+a \nabla_{\beta} l^{\beta}=0
$$

et de même

$$
\nabla_{\beta}\left(b l^{\beta}\right)=l^{\beta} \partial_{\beta} b+b \nabla_{\beta} l^{\beta}=0
$$

$a$ et $b$ étant nuls sur $\Sigma$, il résulte de (13-3) et (13-4) que $a=b=0$ en dehors de $\Sigma$. Par suite le champ électromagnétique est singulier en dehors de $\Sigma$. Nous énoncerons ( ${ }^{4}$ ).

Theorene. - Si un champ électromagnétique de type intégrable est singulier sur une hypersurface $\Sigma$ orientée dans l'espace el satisfait aux équations de Maxwell du vide, il est singulier en dehors de $\Sigma$.

\section{Etude du champ singulier de type intégrable.}

a) Etant donné un champ singulier complétement intégrable sur un voisinage $U$, il existe sur ce voisinage une fonction $\sigma$, definie à la transformation $\sigma \rightarrow f(\sigma)$ près, où $f$ est une fonction arbitraire, telle que II soit défini par l'équation $d \sigma=0$. Il en résulte:

$$
d \sigma \wedge F=0 \quad d \sigma \wedge(* F)=0 .
$$

A la fonction $\sigma$ on donne le non de fonction de phase pour le champ envisagé. Les relations (14-1) caractérisent les champ singuliers de type intégrable. Les variétés $\sigma=$ const. sont des variétés caractéristiques des équations de MAXWELL.

b) Etant donné un champ singulier, rapportons-le à des repères adaptés. Si $\lambda=\theta^{0}-\theta^{1}$, le champ $\Pi$ peut etre défini par l'equation $\lambda=0$. Pour que ce champ soit completement intégrable, il faut et il suffit d'après le théorème de Frobenius que

$$
\lambda \wedge d \lambda=0
$$

(4) Un résultat un peu plus général a đté établi par. L. Marıot. 
La condition (14-2) est équivalente à l'annulation des quatre formes de

$$
\theta p \wedge \lambda \wedge d \lambda=0
$$$$
(\rho=0,1.2,3) \text {. }
$$

Pour $\rho=0$ et 1 on obtient la condition unique:

$$
\theta^{\circ} \wedge \theta^{x} \wedge d \lambda=0 \text {. }
$$

Pour $\rho=2$ ou 3 on obtient deux conditions qui se trouvent identiquement vérifiées en vertu des équations de Maxwell. En effet de (7.3) pris avec $Z=0$ soit :

$$
F=Y \theta^{2} \wedge \lambda \quad * F=-Y \theta^{3} \wedge \lambda
$$

il résulte par dérivation puisque $d F=d * F=0$

$$
d\left(Y \theta^{2}\right) \wedge \lambda-Y \theta^{2} \wedge d \lambda=0 \quad d\left(Y \theta^{3}\right) \wedge \lambda-Y \theta^{3} \wedge d \lambda=0 .
$$

Par produit extérieur par $\lambda$, on voit que (14-3) est satisfait pour $\rho=2$ et 3 . Le fait pour une radiation électromagnétique pure d'être de type intégrable n' introduit qu' une condition scalaire supplementaire.

Si $F$ est singulier de type intégrable.

$$
\nabla_{\alpha} l_{\rho}-\nabla_{\rho} l_{\alpha}=0 \text {. }
$$

De (1.1-8) et (11.9) il résulte alors par addition membre la relation différentielle:

$$
2 l \rho \nabla_{\rho} F_{\alpha \beta}+\left(\nabla_{\rho} l^{\rho}\right) F_{\alpha \beta}=0
$$

relation analogue à (9.11) ou (9.12).

\section{5, Application.}

Donnons-nous sur $U$ une fonction $\sigma$ non constante et un champ électro. magnetique satisfaisant aux équations de MAXweLl du vide. Considérons la 2-forme définie par:

$$
H=f(\sigma) F
$$

où $f$ est une fonction arbitraire non constante. On a manifestement :

$$
* H=f(\sigma)(* F) \text {. }
$$

Par différentiation extérieure, il vient:

$$
d H=f^{\prime}(\sigma) d \sigma \wedge F \quad d(* H)=f^{\prime}(\sigma) d \sigma \wedge(* F) .
$$

Pour que $H$ satisfasse aux équations de Maxwell du vide, il faut et il suffit que:

$$
d \sigma \wedge F=0 \quad d \sigma \wedge * F=0
$$

c' est-à-dire que $F$ définisse un champ singulier de type intégrable admettant la fonction de phase $\sigma$. 
Theoreme. - Etant donnés une fonction $\sigma$ non constante et un champ électromagnétique $F$ satisfaisant aux équations de Maxvell du vide, pour que le champ

$$
H=f(\sigma) F
$$

(f fonction arbitraire non constante) satisfasse aux mêmes équations, il faut et il suffit que $F$ soit un champ singulier de type intégrable admeltant $\sigma$ comme fonction de phase.

Notons que $H$ satisfait alors quel que soit $f$ aux equations de MAxweLL dì vide.

\section{Fronts d'onde gravitationmelle en relativité générale.}

\section{Expressions du tenseur de courbure et du tenseur de Ricci.}

Nous nous proposons, dans cette section, d'étudier la notion de front d'onde gravitationnelle en relativité générale, Au cours de cette étade, le tenseur de courbure de la variété riemannienne $V_{4}$ jouera un rôle essentiel et il nous faut analyser comment les dérivées secondes des potentiels de gravitation par rapport aux coordonnées locales entrent dans l'expression des composantes de ce tenseur. Considérons une variété riemannienne $V_{m+1}$ de signature quelconque. Dans nn voisinage $U$ rapporté à des cordonnées locales $\left(x^{x}\right)$, soient $\Gamma_{\beta_{\gamma}}^{x}$ les coefficients de la connexion riemannienne de $V_{m+1}$ relativement anx coordonnées locales. Par definition le tenseur de courbure de $V_{m+1}$ est le tenseur de composantes:

$$
R_{\beta, \lambda \mu}^{\alpha}=\partial_{\lambda} \Gamma_{\beta_{\beta}}^{z}-\partial_{\mu} \Gamma_{\beta \lambda}^{\alpha}+\Gamma_{\rho \lambda}^{\alpha} \Gamma_{\beta ! \mu}^{\rho}-\Gamma_{\rho \mu}^{x} \Gamma_{\beta \lambda}^{\rho} .
$$

En exprimant les $\Gamma_{\beta \gamma}^{x}$ à l'aide des symboles de Christoffed, il vient :

$$
R_{\beta, \lambda \mu}^{\alpha_{\mu}}=g^{\alpha \rho}\left(\partial_{\lambda}[\beta \mu, \rho]-\partial_{\mu}[\beta \lambda, \rho]\right)+K^{\alpha}, \lambda \mu
$$

où les $K_{\beta, \lambda \mu}^{x}$ ne dépendent que des dérivées premières des potentiels $g_{\rho \sigma}$ et des potentiels eux-mèmes. On en déduit:

$$
R_{\alpha \beta, \lambda \mu}=\partial_{\lambda}[\beta \mu, \alpha]-\partial_{\mu}[\beta \lambda, \alpha]+K_{\alpha \beta, \lambda \mu}
$$

et en développant les symboles de Christoffex:

$$
R_{\alpha \beta, \lambda \mu}=\frac{1}{2}\left(\hat{c}_{\beta \lambda} g_{x \mu}+\partial_{\lambda \mu} g_{\alpha \beta}-\partial_{\alpha \lambda} g_{\beta \mu}-\hat{c}_{\beta \mu} g_{x \lambda}-\partial_{\lambda \mu} g_{\lambda \beta}+\partial_{\alpha \beta} g_{\beta \lambda}\right)+K_{\alpha \beta, \lambda_{\mu}} \cdot
$$

On obtient ainsi :

$$
R_{\alpha \beta, \lambda \mu}=\frac{1}{2}\left(\hat{c}_{\beta \lambda,} g_{x, \mu}+\partial_{x \mu} g_{\beta \lambda}-\hat{\varepsilon}_{\beta,} g_{\alpha \lambda}-\partial_{\alpha \lambda} g_{\beta \mu}\right)+K_{\alpha \beta, \lambda \mu}
$$


Le tenseur de RICCI de $V_{m+1}$ est défini par:

$$
R_{\alpha \beta}=R_{\beta, \sigma \alpha}=g^{\circ \sigma} R_{\rho \beta, \sigma \alpha} \text {. }
$$

Par contraction dans (16.2) des indices $\alpha$ et $\lambda$ et changement du nom des indices, il vient:

$$
R_{\alpha \beta}=\frac{1}{2} g^{\rho \sigma}\left(\partial_{\beta \sigma} g_{\alpha \beta}+\partial_{\alpha \beta} g_{\beta \sigma}-\partial_{\alpha \beta} g_{\rho \sigma}-\partial_{\rho \sigma} g_{\alpha \beta}\right)+K_{\alpha \beta} .
$$

\section{Discontinuités des dérivées secondes des potentiels.}

Supposons un voisinage $U$ de la variété «espace-temps $V_{4}$ rapporté à un système de coordonnées locales $\left(x^{\alpha}\right)$. Le tenseur de gravitation étant supposé $\left(C^{1}, C^{3}\right.$ par morceaux) les dérivées secondes des potentiels de gravitation $g_{a \beta}$ par rapport aux coordonnées locales peuvent admettre à la traversée de certaines hypersurfaces $S$ des discontinuités que nous proposons d'étudier.

Soit done $V_{m+1}$ une variété différentiable de classe $\left(C^{2}, C^{4}\right.$ par morceaux $)$ munie d'une métrique riemannienne de type hyperbolique normal, de classe $\left(C^{1}, C^{3}\right.$ par morceaux). Un voisinage $U$ de $V_{m+1}$ etant rapporté à un système de coordonnées locales $\left(x^{x}\right)$, soit $f\left(x^{x}\right)=0$ l'équation locale d' une hypersurface $S$ à la traversée de laquelle peuvent se produire des discontinuités. Des conditions dé HADAMard sur la propagation des ondés, il résulte qu'il existe aux points de $S$ un système de quantités locales $a_{x \beta}$ tel que les discontinuités $\left[\partial_{\lambda, \mu} g_{a, \beta}\right]$ s'expriment par les formules :

$$
\left[\partial_{\lambda \mu} g_{\alpha \beta}\right]=a_{\alpha \beta} \partial_{\gamma} f \partial_{\mu} f
$$

Soit en désignant par $l_{\lambda}$ le gradient de $f$ :

$$
\left[\partial_{\lambda \mu} g_{\alpha \beta}\right]=a_{\alpha \beta} l_{\lambda} l_{\mu}
$$

Etudions comment le système des $a_{\alpha \beta}$ se transforme dans un changement de coordonnées locales. Si $x^{\alpha}=x^{\alpha}\left(x^{a^{\prime}}\right)$ définit ce changement de coordonnées, on a:

Par dériyation, il vient

$$
g_{\sigma^{\prime} \tau^{\prime}}=A_{\sigma^{\prime}}^{\alpha} A_{\tau}^{\beta} g_{x \beta} \quad\left(A_{\sigma^{\prime}}^{\alpha}=\frac{\partial x^{\alpha}}{\partial x^{\sigma^{\prime}}}\right) .
$$

$$
\partial_{v^{\prime}} g_{\sigma^{\prime} \tau^{\prime}}=A_{\sigma^{\prime}}^{\alpha} A_{\tau^{\prime}}^{\beta} A_{\gamma^{\prime}}^{\lambda} \partial_{\lambda} g_{a \beta}+\left(\partial_{\gamma^{\prime}} A_{\sigma^{\prime}}^{\alpha} \cdot A_{\tau^{\prime}}^{\beta}+A_{\tau^{\prime}}^{\beta} \partial_{y^{\prime}} A_{\tau^{\prime}}^{\beta}\right) g_{\lambda \beta}
$$

Les dérivées troisièmes $\partial_{\nu^{\prime} f^{\prime}} A_{\sigma^{\prime}}^{x}$ pouvant être discontinues à la traversée de $S$, les discontinuités a travers $S$ des dérivées secondes des potentiels de la métrique sont reliées par les formules:

$$
\left.\left[\partial_{\nu^{\prime} \rho} g_{\sigma^{\prime} \tau^{\prime}}\right]=A_{\sigma^{\prime}}^{\alpha} A_{\tau^{\prime}}^{\beta} A_{\nu^{\prime}}^{\lambda} A_{\rho^{\prime}}^{\mu}\left[\partial_{\gamma_{\mu},} g_{\alpha \beta}\right]+\left[\partial_{\nu^{\prime} \rho^{\prime}} A_{\sigma^{\prime}}^{\alpha}\right] A_{\tau^{\prime}}^{\beta}+A_{\sigma^{\prime}}^{\alpha}\left[\partial_{\nu^{\prime} \rho^{\prime}} A_{\tau^{\prime}}^{\beta}\right]\right) g_{\alpha \beta}
$$

Si, conformément aux conditions de HADAMARD, nous posons:

$$
\left[\partial_{v^{\prime} \rho^{\prime}} A_{\sigma^{\prime}}^{z}\right]=t_{\alpha} l_{v^{\prime}} l_{\rho^{\prime}} l_{z^{\prime}}
$$


et :

$$
t_{\beta}=t^{\alpha} g_{\alpha \beta}
$$

il vient:

$$
a_{\sigma^{\prime} \tau}=A_{\sigma^{\prime}}^{\alpha} A_{\tau^{\prime}}^{\beta}\left(a_{\alpha \beta}+t_{\beta} l_{\alpha}+t_{\alpha} l_{\beta}\right)
$$

Ainsi si le changement de coordonnées est de classe $C^{3}$ an voisinage de $S$, les $a_{\alpha \beta}$ se transforment selon la loi tensorielle:

$$
a_{\sigma^{\prime} \tau^{\prime}}=A_{\sigma^{\prime}}^{\alpha} A_{\tau^{\prime}}^{\beta} a_{\alpha \beta} .
$$

Si le changement de coordonnées envisagé est tangent le long de $S$ au chan. gement identité et admet des dérivées troisièmes discontinues à la traversée de $S$, les $a_{x \beta}$ subissent la transformation:

$$
a_{\alpha \beta} \rightarrow a_{\alpha \beta}+t_{\alpha} l_{\beta}+t_{\beta} l_{\alpha} .
$$

La formule (17.5) peut être considérée comme résultant de la composition de $(17.7)$ et $(17.6)$.

En tenant compte de la formule (17.2), on obtient a partir de (16.2):

$$
\left[R_{\alpha \beta . \lambda \mu}\right]=\frac{1}{2}\left(a_{\alpha \mu} l_{\beta} l_{\lambda}+a_{\beta \beta \lambda} l_{x} l_{\mu}-a_{\alpha \lambda} l_{\beta} l_{\mu}-a_{\beta \mu} l_{\alpha} l_{\lambda}\right) .
$$

Les $R_{\alpha \beta, \lambda \mu}$ définissant un tenseur, il en est de même des $\left[R_{\alpha \beta, \lambda, \mu}\right]$ et la formale (17.8) est nécessairement invariante par la transformation (17.7) comme on le vérifie aisément.

En ce qui concerne le tenseur de Riccr, on a, d'après (16.4):

$$
\left[R_{\alpha \beta}\right]=\frac{1}{2} g^{g \sigma}\left(a_{\alpha \beta} l_{\beta} l_{\sigma}+a_{\beta \sigma} l_{\alpha} l_{\rho}-a_{\rho \sigma} l_{x} l_{\beta}-a_{\alpha \beta} l_{\rho} l_{\sigma}\right) \text {. }
$$

\section{Variétés caractéristiques des équations d'Einstein}

a) Supposons que la métrique de la variété $V_{m+1}$ satisfasse à des «équations d'Einstein » généralisées de la forme

$$
S_{\alpha \beta}=\chi T_{\alpha \beta}
$$

où $T_{\alpha \beta}$ est un tenseur symétrique donné, supposé continu dans le domaine envisagé, $\chi$ une constante et où $S_{\alpha \beta}$ désigne le tenseur

$$
S_{x \beta}=R_{\alpha \beta}-\frac{1}{2} g_{\alpha \beta} R \text {. }
$$

De (18.2) on déduit par contraction:

$$
S=R-\frac{m+1}{2} R=-\frac{m-1}{2} R .
$$


Il en résulte pour $m \neq 1$ :

$$
R_{\alpha \beta}=S_{\alpha \beta}-\frac{1}{m-1} g_{\alpha \beta} S \text {. }
$$

De la continuité de $T_{\alpha \beta}$, on déduit d'après (18-1) $\left[S_{\alpha \beta}\right]=0$ et par suite en vertu de (18-3) et de la continuité de la métriquo

$$
\left[R_{x \beta}\right]=0 \text {. }
$$

b) Chisissons un'système de coordonnées locales telles que l'hypersur. face $S$ à la traversée de laquelle nous étudions les discontinuités ait pour équation locale $x^{0}=0 ; l_{x}$ admet dans ce cas pour composantes

$$
l_{0}=1 \quad l_{u}=0 \quad(u, v, \text { etc. } \ldots=1,2, \ldots, m) .
$$

Les seules dérivées secondes qui peuvent être discontinuites à la traversée de $S$ sont les $\partial_{00} g_{\alpha \beta}$ et l'on a

$$
\left[\partial_{00} g_{u v}\right]=a_{u v} \quad\left[\partial_{00} g_{0 x}\right]=a_{0 x}
$$

où les $a_{u v}$ sont invariants par la transformation (17.7) et où les $a_{0 x}$ se transforment selon

$$
a_{0 x} \rightarrow a_{0 x}+t_{a x}+t_{0} l_{x} .
$$

Le choix des $t_{x}$ ou des $t^{x}$ permet d'annuler ou de faire apparaître des discontinuités des $\partial_{00} g_{0 x}$. De plus, d'après (17-9), il vient:

$$
\begin{aligned}
& {\left[R_{u v}\right] \equiv-\frac{1}{2} g^{00} a_{u v}=0} \\
& {\left[R_{0 u}\right] \equiv \frac{1}{2} g^{0 v} a_{u v}=0} \\
& {\left[R_{00}\right] \equiv-\frac{1}{2} g^{u v} a_{u v}=0}
\end{aligned}
$$

et les premiers membres de (18-6), (18.7), (18-8) ne font pas intervenir les $\left[\partial_{00} g_{0 x}\right]$. Nous dirons que les dérivées $\partial_{00} g_{u v}$ sont significatives relativement à l'hypersurface et que les $(m+1)$ dérivées $\partial_{00} g_{0 x}$ sont non significatives. C' est le cas où les dérivées significatives présentent des discontinuités qu' il convient d'examiner.

Si toutes les discontinuités des dérivées significatives ne sont pas nulles il existe un $a_{u v} \neq 0$ et de (18.6) il résulte

$$
g^{00}=0 \text {. }
$$

Le vecteur $l^{*}$ admet alors les composantes:

$$
l^{0}=0 \quad l^{u}=g^{0 u}
$$


et d'apres (18.7), il satisfait aux relations

$$
a_{u v} l^{v}=0 \text {. }
$$

De plus d'après (18-8)

$$
a=g^{\alpha \beta} a_{\alpha \beta}=2 g^{0 u} a_{0 u}=2 a_{0 u} l^{u} .
$$

En coordonnées locales arbitraires. (18.9) s'ecrit:

$$
g^{\times \beta} l_{\alpha} l_{\beta}=0
$$

et le gradient de $f$ est de longuenr nulle. Ainsi $S$ est nécessairement solution de l'équation aux dérivées partielles du premier ordre:

$$
\Delta_{1} f \equiv g^{\alpha \beta} \partial_{\alpha} f \partial_{\beta} f=0
$$

c'est-à-dire, pour le cas de la relativité générale, de (9-4).

Ainsi dans ce cas $(m=3)$, les fronts d'onde gravitationnelle, ou variétés carnctéristiques des équations d'ErNSTEIN sont les hypersurfaces tangentes en ehacun de leurs points au cône élémentaire en ce point. Les rayons gra* vitationnels, trajectoires du champ de vecteurs défini par $\vec{l}$ sur une telle hypersurface, sont toujours des géodésiques de longueur nulle.

Revenons au cas général d'une variété $V_{m+1}$. Les équations (18-10) et (18-11) peuvent se traduire en coopdonnées locales arbitraires par la relation

$$
a_{\alpha \beta} l^{\beta}=\frac{a}{2} l_{\alpha} \quad\left(a=g^{\alpha \beta} a_{\alpha \beta}\right) .
$$

On voit en particulier que le vecteur $l *$ de longueur nulle est vecteur propre de la matrice $\left(a_{\alpha \beta}\right)$. On vérifiera immédiatement que (18-12) est invariant par la transformation (17.7).

Inversement, si $l^{*}$ est de longueur nulle et satisfait à (18-12), on a $\left[R_{\alpha \beta}\right]=0$.

\section{Lemme sur les variétés admettant un gronpe d'isométries. Cas pentadimensionnel.}

On sait que la considération d'une variété pentadimensionnelle munie d'un groupe d'isométries à un paramètre permet d'unifier géométriquement champ gravitationnel et champ électromagnétique (en l'absence d'induction). Nous allons plus généralement rappeler des formules relatives à nne variété $V_{m+1}$ admettant un groupe à 1 paramètre d'isométries.

a) Considérons une variété différentielle $V_{m+1}$, de dimension $(m+1)$, satisfaisant aux mêmes hypothèses de différentiabilité que la variété espacetemps, c'est-à-dire $\left(C^{2}, C^{4}\right.$ par morceaux). Sur cette variété, nous supposons définie une métrique riemannienne $d \sigma^{2}$ de type hyperbolique normal à 1 carré 
positif et $m$ carrés négatifs et de classe $\left(C^{1}, C^{3}\right.$ par morceanx). Nous supposons, au cours de ce paragraphe, que les indices grecs prennent les valeurs $0,1, \ldots, m$ et les indices latins les valeurs $1,2, \ldots, m$. En coordonnées locales la métrique s'écrit :

$$
d \sigma^{2}=\gamma_{s \beta} d x^{\gamma} d x^{\beta}
$$

et sur un voisinage, elle peut être décomposée en la somme algébrique de carrés :

$$
d \sigma^{2}=\left(\theta_{-m}^{m}\right)^{2}-\left(\theta_{-}^{0}\right)^{2}-\left(\theta_{-}^{1}\right)^{2}-\ldots-\left(\theta^{m+1}\right)^{2}
$$

où les $\theta \underline{x}$ sont des formes de Pfafr locales linéairement indépendantes. Par dualité le vóisinage envisagé de $V_{m+1}$ se trouve muni de repères orthonormés.

Nous supposons que $V_{m+1}$ admet un groupe connexe à 1 paramètre d'isométries globales, à trajectoires orientées $d \sigma^{2}<0$, ne laissant invariant aucun point de $V_{n+1}$ et jouissant de la propriété suivante: par passage an quotient par la relation d'équivalence définie par le groupe d'isométries, on obtient une variété différentiable $V_{m}$ de classe $\left(C^{2} . C^{4}\right.$ par morceaux); les points $z$ de $V_{m}$ peuvent être identifiés aux différentes trajectoires du groupe dans $V_{m+1}$.

Soit $\vec{\xi}$. le vecteur générateur infinitésimal du groupe d'isométries; aucun point de $V_{m+1}$ n'étant invariant, $\vec{\xi}$ ne s'annule pas. Il satisfait aux équations de KILLING

$$
L(\vec{\xi}) g_{\alpha \beta}=\nabla_{\beta} \xi_{\alpha}+\nabla_{\alpha} \xi_{\beta}=0 .
$$

Soit $\left(x^{i^{\prime}}\right)$ un système de coordonnées locales arbitraires de $V_{m}$. Un point $x$ de $V_{m+1}$ peut être repéré par sa trajectoire $z(x)$ et sur cette trajectoire par une coordonnée $\left(x^{0^{\prime}}\right)$. Dans les coordonnées locales $\left(x^{\lambda^{\prime}}\right)$ les trajectoires $d u$ champ de vecteurs $\vec{\xi}$ sont les lignes $x^{i^{\prime}}=$ const. et l'on a :

$$
\xi^{i^{\prime}}=0 \quad \xi^{\prime} \neq 0 .
$$

Effectuons le changement de coordonnées locales défini par:

$$
x^{i}=x^{i^{\prime}} \quad x^{0}=f\left(x^{0^{\prime}}, x^{j^{\prime}}\right)
$$

On peut choisir $f$ de façon que la nouvelle composante

Il suffit de prendre:

$$
\xi^{0}=A_{0}^{0}, \xi^{o^{\prime}}=\frac{\partial f}{\partial x^{0^{\prime}}} \xi^{0^{\prime}}=1
$$

$$
\frac{\partial f}{\partial x^{0^{\prime}}}=\frac{1}{\xi^{0^{\prime}}} \quad\left(\xi^{0^{\prime}} \neq 0\right)
$$

et la fonction $f$ se trouve définie à une fonction additive près de coordonnées $\left(x^{j^{\prime}}\right)$. Dans le système de coordonnées $\left(x^{2}\right)$ ainsi défini

$$
\xi^{i}=0 \quad \xi^{0}=1
$$


$x^{\lambda}$ est dit adapté au groupe d'isométries envisagé. Dans ce système $\xi_{x}=g_{x 0}$ et par suite $\nabla_{\beta} \xi_{x}=[\beta 0, \alpha]$. Par suite les équations de KILLING (19-3) se traduisent par:

$$
\partial_{0} \gamma_{\alpha \beta}=0 \text {. }
$$

Les systèmes de coordonnées locales adaptés au groupe d'isométries sont definis au changement près:

$$
x^{i^{\prime}}=\psi^{i^{\prime}\left(x^{i}\right)} \quad x^{0^{\prime}}=x^{0}+\psi\left(x^{j}\right)
$$

où $\psi$ sont des fonctions arbitraires des $x^{j}$.

A chaque point $x$ d' un voisinage de $V_{m+1}$ associons un repère orthonormé dont le premier vecteur soit tangent en $x$ à la trajectoire passant par ce point. Un tel repère est dit adapté au groupe 'd'isométries. Relativement aux repères adaptés, il vient :

$$
d \sigma^{2}=-\left(\theta_{-}^{0}\right)^{2}+\left(\theta_{-m}^{m}\right)^{2}-\left(\theta^{1}\right)^{2}-\ldots-\left(\theta_{-m-1}^{m}\right)^{2}
$$

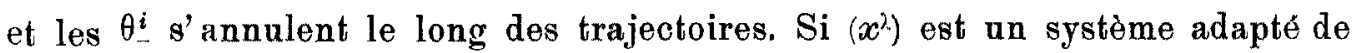
coordonnées locales, les $\theta^{i}$. sont des formes de PFAfF par rapport aux $d x^{i}$ et (19.6) n' est autre que la décomposition en carrés de $d \sigma^{2}$, la variable $d x^{0}$ jouant le ròle de variable directrice. On en déduit:

$$
d \sigma^{2}=-\left(\theta^{0}\right)^{2}+d s^{2}
$$

avec

$$
\theta_{-}^{0}=\frac{1}{\sqrt{\left|\gamma_{00}\right|}}\left(\gamma_{00} d x^{0}+\gamma_{0 i} d x^{i}\right)
$$

et

$$
d s^{2}=\left(\theta_{-m}\right)^{2}-\left(\theta^{1}\right)^{2}-\ldots-\left(\theta^{m-1}\right)^{2}=\left(\gamma_{i j}-\frac{\gamma_{0 i} \gamma_{0 j}}{\gamma_{00}}\right) d x^{i} d x^{j} .
$$

De (19.9), il résulte que $d s^{2}$ est indépendant du système de coordonnées locales adaptées choisi et, dans ce système, est indépendant de $x^{0}$. La forme quadratique $d s^{2}$ détermine sur $V_{m}$ une métrique riemannienne de type hyperbolique, la métrique quotient, de coefficients

$$
g_{i j}=\gamma_{j j}-\frac{\gamma_{0 i} \gamma_{0 j}}{\gamma_{00}}
$$

b) Le carré du vecteur $\vec{\xi}$ est strictement négatif et nous le désignerons par $-\xi^{2}$. En coordonnées adaptées $\gamma_{00}=-\xi^{2}$ et $\xi$ définit sur $V_{m}$ un champ scalaire. 
A. LICHNerowicz: Ondes et radiations électromagnétiques, etc.

Considérons maintenant le vecteur $\varphi_{a}$ de $V_{m+1}$ colinéaire à $\xi_{a}$ et défini par

$$
\beta \varphi_{\alpha}=\frac{\xi_{\alpha}}{-\xi^{2}}
$$

où $\beta$ désigne une constante convenable. En coordonnées adaptées

$$
\beta \varphi_{i}=\frac{\gamma_{0 i}}{\gamma_{00}} \quad \beta \varphi_{0}=1
$$

Considérons le rotationnel $F_{\alpha \beta}$ de $\varphi_{\alpha}$. D' après (19-12)

$$
F_{0 z}=\partial_{0} \varphi_{\alpha}-\partial_{\alpha} \varphi_{0}=0
$$

D'autre part si l'on effectue le changement de coordonnées locales adaptées défini par $x^{i \prime}=x^{i}, x^{0^{\prime}}=x^{0}+\psi\left(x^{i}\right)$

$$
\varphi_{i}=\varphi_{i^{\prime}}+\frac{1}{\beta} \frac{\partial \psi}{\partial x^{i}}
$$

et par suite $F_{i j}=F_{i^{\prime} j^{\prime}}$. Ainsi les $F_{i j}$ définissent sur $V_{m}$ un tenseur antisymétrique.

Il est aisé de voir la signification géométrique de la nullité du tenseur $F_{i j}$. S'il est possible de trouver, pour un voisinage $U$ de $V_{m}$, des coordonnées locales adaptées telles que les trajectoires correspondant à $U$ soient trajectoires orthogonales des hypersurfaces $x^{0}=$ const., on a dans ce système $\xi_{i}=\gamma_{o i}=0, \varphi_{i}=0$ et par suite $F_{i j}=0$. Inversement si $F_{i j}=0$, le tenseur $F_{\lambda \mu}$ de $V_{n+1}$ est nul et $\left(\beta \varphi_{0}=1, \beta \varphi_{i}\right)$ définit localement un champ de gradient. Il existe donc, dans un voisinage $U$ de $V_{m}$, une fonction $\psi\left(x^{i}\right)$ telle que:

$$
\beta \varphi_{\alpha}=\partial_{\alpha}\left[x^{0}+\psi\left(x^{i}\right)\right]
$$

Ainsi $F_{i j}=0$ exprime qu'il existe des coordonnées locales adaptées telles que les trajectoires da groupe correspondant à un voisinage $U$ soient trajectoires orthogonales $d^{\prime}$ hypersurfaces $x^{0}=$ const.

c) Sur $V_{m}$ nous avons ainsi défini, à partir de la métrique de $V_{m+1}$ et du groupe d'isométries, une métrique (19-10), un scalaire $\xi$ et un tensenr antisymetrique $F_{i j}$.

Rapportons $V_{m+1}$ à des repères orthonormés adaptés. D'après (19.9) la variété $V_{m}$ considérée comme variété riemannienne pour la métrique-quotient se trouve ainsi rapportée à des repères orthonormés. On peut exprimer les composantes $R_{a \beta, \gamma \delta}$ du tenseur de courbure de $V_{m+1}$ en fonction des composantes du tenseur de courbure de $V_{m}$, de $\xi$ et des eomposantes du tenseur antisymétrique $F$. 
On démontre en effet les formules suivantes $\left({ }^{(}\right)$:

$$
\begin{aligned}
& R_{i j, k l}=\stackrel{*}{R}_{\underline{i j}, k l}+\frac{\beta^{2} \xi^{2}}{4}\left(F_{\underline{i k}} F_{\underline{j l}}-F_{\underline{i l}} F_{j k}\right)+\frac{\beta^{1} \xi^{2}}{2} F_{\underline{i j}} F_{k l}
\end{aligned}
$$

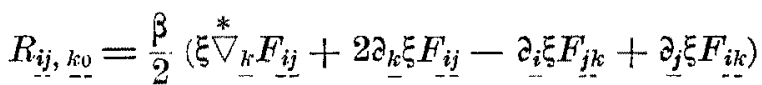

$$
\begin{aligned}
& R_{\underline{i_{0}, k_{0}}}=\frac{1}{\xi} \stackrel{*}{\nabla}_{\underline{k}}\left(\partial_{i} \xi\right)+\frac{\beta^{2} \xi^{2}}{4} F_{\underline{i}}, F_{\underline{k}} \underline{r}
\end{aligned}
$$

où les éléments relatifs à la métrique $d s^{2}$ ont été munis d'une *.

d) Plaçons-nous dans le cas pentadimensionnel $(m=4)$. Pour les théories JoRDAN-Thiry et de KaLuza-KLEIN les hypothèses précédentes sont satisfaites et le tensenr antisymétrique $F_{i j}$ peut être interprété come le champ électromagnétique.

Des formules précédentes, il résulte qu'à la traversée d' une hypersurface $S$, les discontinuités des composantes du tenseur de courbure de $V_{5}$ peurent s'écrire :

$$
\begin{aligned}
& {\left[R_{i j, k l}\right]=\left[\stackrel{*}{R}_{i j, k l}\right]} \\
& {\left[R_{\underline{i j}, k \mathrm{o}}\right]={ }_{2}^{\beta} \xi\left[{ }^{*}{ }_{\underline{k}} F_{i j}\right]} \\
& {\left[R_{\underline{\text { in } k \text { ko }}}\right]=\frac{1}{\xi}\left[\stackrel{*}{\nabla_{\underline{k}}}\left(\partial_{-} \xi\right)\right]}
\end{aligned}
$$

(19.18) montre que l'étude des discontinuités des dérivées premières $\left[\stackrel{*}{\nabla}_{\underline{\underline{k}}} F_{i]}\right] \mathrm{du}$ champ électromagnétique est équivalente à celle des discontinuités dés composantes $R_{i j, k_{0}}$ du tensenr de courbure de $V_{s}$.

Nous sommes ainsi conduits. pour analyser la structure des ondes oravitationnelles dans l' espace-temps à étudier les discontinuités des composantes du tenseur de courbure de la métrique de la relativité générale.

\section{Formules relatives aux discontinuités du tenseur de courbure pour} $V_{m+1}$.

Revenons au cas d' une variété riemannieune $V_{m+1}$ munie d' nne métrique riemannienne de type hyperbolique normal à un carré positif et $m$ carrés négatifs.

(5) Voir LICHNEROWICz. Théories relativistes de la gravitation et de l'électromagnétisme, p. 119 ; les formules ont été transformées compte-tenu de la signature de la métrique et de l'orientation des trajectoires. 
a) De le formule (17-8), il est possible de déduire une relation intéres: sante entre $l_{\zeta}=\partial_{\varphi} f$ et le tenseur $\left[R_{\left.\alpha \beta, \lambda_{\mu}\right]}\right]$ de discontinuité du tenseur de courbure à la traversée de l' hypersurface $S$ d' équation locale $f\left(x^{\alpha}\right)=0$. On a :

$$
l_{\mu}\left[R_{\alpha \beta, \lambda_{\mu}}\right]=\frac{1}{2}\left(a_{\alpha \mu} l_{\beta} l_{\gamma}-a_{\beta \mu} l_{\gamma} l_{\alpha}\right) l_{\lambda}+\frac{1}{2}\left(a_{\beta \lambda} l_{\gamma} l_{\alpha}-a_{\alpha \lambda} i_{\beta} l_{\gamma} l_{\mu} .\right.
$$

Par permutation circulaire des indices $\alpha, \beta, \gamma$, il vient:

$$
l a\left[R_{\beta \gamma, \lambda \mu}\right]=\frac{1}{2}\left(a_{\beta \mu} l_{\gamma} l_{\alpha}-a_{\gamma \mu} l_{\alpha} l_{\beta}\right) l_{\lambda}+\frac{1}{2}\left(a_{\gamma \lambda} l_{\alpha} l_{\beta}-a_{\beta \lambda} l_{\gamma} l_{\alpha}\right) l_{\mu}
$$

et

$$
l_{\beta}\left[R_{\gamma \alpha}, \lambda_{\mu}\right]=\frac{1}{2}\left(a_{\gamma \mu} l_{\alpha} l_{\beta}-a_{\alpha \mu} l_{\beta} l_{\gamma}\right) l_{\lambda}+\frac{1}{2}\left(a_{\alpha \lambda} l_{\beta} l_{\gamma}-a_{\gamma \lambda} l_{\alpha} l_{\beta}\right) l_{\mu}
$$

Par addition membre à membre de (20-1), (20-2), (20.3) nous obtenons les relations :

$$
l_{\alpha}\left[R_{\beta \gamma, \lambda \mu}\right]+l_{\beta}\left[R_{\gamma \alpha, \lambda \mu}\right]+l_{\gamma}\left[R_{\alpha \beta, \lambda \mu}\right]=0
$$

b) Supposons de plus que la métrique de $V_{m+1}$ satisfasse aux «équations d'EINstein généralisées avec un tenseur second membre continu.

A la traversée de l'hypersurface

$$
\left[R_{\alpha \beta}\right]=0
$$

et d'après l'étude du $\S 18$, le vecteur $l^{\alpha}$ satisfait à la relation (18-12).

De (17.8) on déduit :

$$
l \alpha\left[R_{\alpha \beta, \lambda \mu}\right]=\frac{1}{2}\left(a_{\alpha \mu} l \alpha l_{\beta} l_{\lambda}-a_{\lambda \lambda} l \alpha l_{\beta} l_{\mu}\right)+\frac{1}{2}\left(a_{\beta} l_{\mu}-a_{\beta \mu} l_{\lambda}\right) l_{\alpha} l^{\alpha}
$$

$l^{x}$ étant de longuear nulle, le deuxième terme du second membre est nul. De plus d'après (18-12)

$$
a_{\alpha \mu} l^{\alpha} l_{\lambda}-a_{\alpha \lambda} l^{\alpha} l_{\mu}=\frac{a}{2}\left(l_{\lambda} l_{\mu}-l_{\mu} l_{\lambda}\right)=0 .
$$

Ainsi (20-5) entraîne

$$
l_{x}\left[R_{\approx \beta, \lambda} \lambda_{\mu}\right]=0 .
$$

c) On peut aussi établir directement les relations (20-4) et (20-6) de la manière suivante: les coefficients $I$ de la connexion riemannienne en coordonnées locales étant continues à la traversée de $S$, il résulte des conditions de HaDAmard qu'il existe un système de quantités $u_{\beta \mu}^{x}$ telles que:

$$
\left[\partial_{\lambda} \Gamma_{\beta \mu}^{\alpha}\right]=u_{\beta_{\mu}}^{\alpha} l_{\lambda} \quad\left(l_{\lambda}=\partial_{\lambda} f\right)
$$


De $(16-1)$ il résulte:

$$
\left[R^{x}{ }_{\beta, \lambda \mu}\right]=u_{\beta \mu}^{\alpha} l_{\lambda}-u_{\beta \lambda}^{\alpha} l_{\mu} .
$$

Par multiplication par $l_{v}$ et permutation circulaire sur $\lambda, \mu, \gamma$ il vient:

$$
l_{\lambda}\left[R_{\beta, \mu \nu}^{\alpha_{\beta}}\right]+l_{\mu}\left[R_{\beta, \nu \lambda}^{\alpha}\right]+l_{\nu}\left[R_{\beta, \lambda_{\mu}}^{\alpha}\right]=0
$$

qui est équivalent ì (20-4).

Si la relation (20.5) est satisfaite, on déduit de (20-7):

$$
u_{\beta \mu}^{\rho} l_{\rho}-u_{\beta \rho}^{\rho} l_{\mu}=0
$$

En multipliant (20-7) par $l_{\alpha}$, il vient ainsi :

$$
l_{\alpha}\left[R_{\beta, \lambda_{\mu}}^{x_{1}}\right]=u_{\beta \rho}^{p} l_{\lambda} l_{\mu}-u_{\beta \rho}^{\rho} l_{\lambda} l_{\mu}=0
$$

c'est-à-dire (20-6). Introduisons les formes de courbure:

$$
\mathbf{Q}_{\beta}^{\alpha}=\frac{1}{2} R_{\beta, \lambda_{\mu}{ }^{\alpha}}^{\lambda} \wedge \theta^{\mu} .
$$

Aux discontinuités du tenseur de courbure, nous associons les 2-formes définies aux points de $S$.

$$
\left[\mathbf{Q}_{\beta}^{\alpha}\right]=\frac{1}{2}\left[R_{\beta, \lambda \mu}^{\alpha}\right]^{\theta^{\lambda}} \wedge \theta^{\mu}
$$

Les relations (20-6) et (20-8) expriment que toutes les formes locales $\left[\Omega_{\beta}^{\chi}\right]$ sont singulières, le vecteur nécessairement isotrope $l_{x}$ étant vecteur propre isotrope commun.

\section{Cas général d'un tenseur admettant le type de symétrie d'un tenseur} de courbure.

Au point $x$ de le varieté riemannienne $V_{m+1}$ admettant une métrique de type hyperbolique normal, considérons un tenseur $H_{\alpha \beta, \lambda \mu}(\neq 0)$ jouissant des mèmes propriétés de symétrie que le tenseur de courbure:

$$
H_{\alpha \beta . \lambda \mu}=-H_{\beta \alpha . \lambda \mu}=-H_{\alpha \beta, \mu \lambda} \quad H_{\alpha \beta, \lambda \mu}=H_{\lambda \mu, \alpha \beta} .
$$

Supposons qu'il existe un vecteur $l_{x}$ tel que soient satisfaites les relations:

$$
l_{x} H_{\beta, \gamma \mu}+l_{\beta} H_{\gamma, \lambda \mu}+l_{\gamma} H_{x \beta, \lambda_{\mu}}=0
$$

et

$$
l^{\times} H_{\alpha \beta, \lambda \mu}=0 .
$$

Si nous posons:

$$
\square_{\lambda, \mu}=\frac{1}{2} H_{\alpha \beta, \lambda, \mu} A^{x} \wedge \theta^{\beta}
$$


les relations (21.2) et (21-3) expriment que les formes $\Pi_{\lambda \hat{\mu}}$ sont toutes singulières et admettent le vecteur $l_{\alpha}$, nécessairement isotrope d'après $\$ 7$, comme vecteur propre isotrope commun.

Ainsi le vecteur $l_{\alpha}$ envisagé est nécessairement isoirope.

Nous nous proposons d'étudier la structure du tenseur contracté:

$$
H_{a \beta}=g^{\rho \sigma} H_{a p . \beta \sigma} \text {. }
$$

En contractant dans (21.2) les indices $\alpha$ et $\lambda$, il vient, compte tenu des pro. priétés de symétrie:

$$
l^{\alpha} H_{\alpha \mu, \beta \gamma}+l_{\gamma} H_{\beta \mu}-l_{\beta} H_{\gamma \mu}=0
$$

qui n' est conséquence que de (21.2).

En tenant compte de (21.3) dans (21.6), il vient:

$$
l_{\alpha} H_{\beta \mu}-l_{\beta} H_{\gamma^{\mu}}=0 \text {. }
$$

Si $\nu^{\mu}$ est un vecteur arbitraire en $x$ :

Il en résulte

$$
l_{\gamma} H_{\beta \mu} \nu^{\mu}-l_{\beta} H_{\gamma_{\mu}} \nu^{\mu}=0 \text {. }
$$

$$
H_{\beta \mu} \nu^{\mu}=\lambda(\vec{v}) l_{\beta}
$$

où $\lambda(\vec{v})$ est une forme linéaire en $\vec{v}$. Ainsi il existe un scalaire $\tau$ tel que:

$$
H_{\alpha \beta}=\tau l_{\alpha} l_{\beta} \text {. }
$$

b) Inversement supposons que le tenseur $H_{\alpha \beta, \lambda \mu}$ admettant les propriétés (21.1) et le vecteur $l_{\alpha}$ satisfassent aux relations (21-2) et (21-8). Nous avons vu que (21.2) entrainait (21-6). En tenant compte de (21-8) on obtient ainsi :

$$
l^{\alpha} H_{\alpha ; \mu, \beta \gamma}=0
$$

c' est-à-dire (21-3) et $l^{\alpha}$ est nécessairement isotrope. Nous énoncerons :

THÉORÈme. - Etant donnés. en un point $x$ d'une variété riemannienne $V_{m+1}$ admettant une métrique hyperbolique normale, un tenseur $H_{\alpha \beta, \lambda \mu}$ jouissant des propriétés de symétrie (21-1) et un vecteur $l_{\alpha}$, pour que ces éléments soient liés par les relations (21.2), (21.3) et (21.8) il faut et il suffit que soient sati. sfaites soit les relations (21.2) et (21.3), soit les relations (21-2) et (21-8); $\vec{l}$ est alors isotrope.

c) Les indices $\lambda$ et $\mu$ étant fixés, la 2-forme $\Pi_{\lambda \mu}$ en $x$ est singulière de vecteur fondamental isotrope $\vec{l}$. En introduisant la base $\varphi^{(i)}$ des 2-formes singulières de vecteur fondamental $\vec{l}$ défini au $\$ 7$, il vient:

$$
H_{\alpha \beta, \lambda \mu}=\sum_{i} a_{i(\lambda \mu)} \varphi_{a \beta}^{(i)} \quad(i=1, \ldots,(m-1)) .
$$


Pour $i$ fixé, les $a_{i\left(\lambda_{i, k}\right)}$ definissent encore ane 2-forme singulière de vecteur fondamental $l$ et:

$$
a_{i(\lambda \mu)}=\underset{j}{\Sigma} a_{i j} \varphi_{\lambda \mu}^{(j)}
$$

Il en résulte:

$$
H_{\alpha \beta, \lambda \mu}=\underset{i_{1} j}{\Sigma} a_{i j} \varphi_{\alpha \beta}^{(1)} \varphi_{\lambda \mu}^{(i)}
$$

et daprès la propriété de symmétrie $H_{x \beta, \lambda \mu}=H_{\lambda, \mu, \alpha \beta}$, il vient $a_{i j}=a_{i j}$. Ainsi:

$$
H_{\alpha \beta, \lambda \mu}=\underset{i_{1 j} j}{\mathbf{v}} a_{i j}\left(l_{\alpha} n_{\beta}^{(i)}-l_{\beta} n_{x}^{(i)}\right)\left(l_{\lambda} n_{1}^{(j)}-l_{\mu} n_{\lambda}^{(j)}\right) .
$$

Par contraction on obtient:

$$
H_{x \beta}=-\Sigma\left(a_{i i}\right) l_{\alpha} l_{\beta}
$$

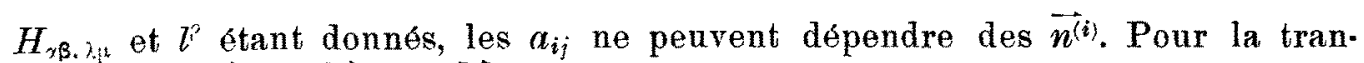
sformation $\overrightarrow{n^{(i)}} \rightarrow \overrightarrow{n^{(i)}}+k^{(i)} \vec{l}$, les formes $\varphi^{(i)}$ et par suite les $a_{i j}$ sont invariants: pour une rotation du système des $\vec{n}^{(i)}$ dans le $(m-1)$-plan qu'ils déterminent, les $a_{i j}$ sont les composantes d'un tenseur symétrique. En particulier, lorsque $H$ est donné, il est possible de choisir pour vecteurs $n^{(i)}$ un système de vecteurs propres de la matrice $\left(a_{i j}\right)$ par rapport à la matrice unité et ainsi d'annuler $a_{i j}$ pour $i \neq i$.

Si nous introduisons les quantités symétrique:

$$
b_{\alpha \lambda}=\Sigma a_{i j} n_{x}^{(i)} n_{\lambda}^{(j)}
$$

il vient selon $(21-9)$ :

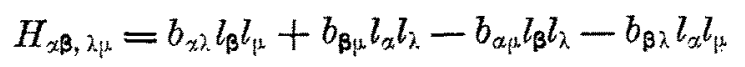

et l'on a manifestement:

$$
b_{x, 2} l^{\lambda}=0
$$

Ainsi il existe des quantités $b_{n \lambda}$ satisfaisant (21-13) et telles que le tenseur $H_{x \beta, \lambda_{\mu}}$ admette l'expression (21-12). Cherchons à quelle transformation près ces quantités sont définies: à cet effet multiplions (21-12) par $v^{\beta}$, où $v^{\beta}$ désigne un vecteur arbitraire. Si le tenseur $H$ est nul, il vient:

$$
\left\{\left(v^{\beta} l_{\beta}\right) b_{\alpha \lambda}-v^{\beta} b_{\beta \lambda} l_{\alpha}\right) l_{\mu^{\mu}}-\left\{\left(v^{\beta} l_{\beta}\right) b_{\alpha \mu}-v^{\beta} b_{\beta \mu} l_{\alpha}\right\} l_{\lambda}=0 .
$$

Il en résulte qu'il existe des quantités $t_{\alpha}$ telles que:

$$
\left(v^{\beta} l_{\beta}\right) b_{\alpha \lambda}-v^{\beta} b_{\beta \lambda} l_{\alpha}=t_{\alpha} l_{\lambda}\left(v^{\beta} l_{\beta}\right)
$$


Ainsi les $b_{\alpha \lambda}$ ont nécessairement la forme:

$$
b_{\alpha \lambda}=t_{\alpha} l_{\lambda}+u_{\lambda} l_{\alpha}=t_{x} l_{\lambda}+t_{\lambda} l_{\alpha}+\left(u_{\lambda}-t_{\lambda}\right) l_{\alpha} .
$$

D'après la symétrie de $b_{\alpha \lambda} ; u_{\lambda}-t_{\lambda}$ est colinéaire à $l_{\lambda}$ et peut être annulè par modification de $t_{\alpha}$. En tenant compte de (21-13), on voit que les $b_{a \lambda}$ satisfaisant à (21.12), (21.13) sont définis à la transformation près:

$$
b_{\alpha \lambda} \rightarrow b_{\alpha \lambda}+t_{\alpha} l_{\lambda}+t_{\lambda} l_{\alpha}
$$

$\left(\operatorname{avec} t_{\alpha} l^{\alpha}=0\right)$

On notera que le scalaire

$$
e=b_{\alpha h} b^{\alpha \lambda}
$$

ne dépend en vertu de (21-14) que du tenseur $H$ et du choix du vecteur $\vec{l}$. De plus si les $b_{a \lambda}$ sont définis à partir de (21-11), on a:

$$
e=\sum_{i_{1 j}}\left(\alpha_{i j}\right)^{2} .
$$

Ainsi $e$ est strictement positif et ne s'annule que si $H=0$.

d) Plaçons-nous dans le cas $m=3$ de la relativité générale. Soit $\overrightarrow{e_{0}}$ un vecteurs normé orienté $d s^{2}>0, \overrightarrow{e_{1}}$ un vecteur normé orthogonal à $\overrightarrow{e_{0}}$ et tel qu'on puisse prendre

$$
\vec{l}=\overrightarrow{e_{0}}+\vec{e}_{1} .
$$

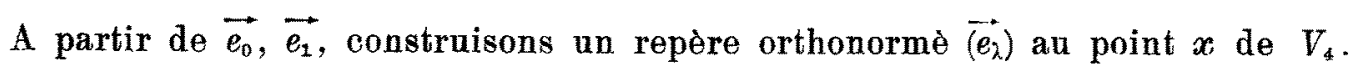
Nous désignons ici par $u, v \ldots$ des indices prenant les valeurs 1, 2, 3, par $A, B, \ldots$ des indices prenant les valeurs 2,3 . Dans ce repère les relations (21.3) se traduisent par:

$$
H_{0 \beta, \lambda \mu}+H_{1 \beta, \lambda \mu}=0 \text {. }
$$

Dans (21-2) faisons $\alpha=A, \beta=B, \gamma=0$. Il vient:

$$
H_{A B, \lambda \mu}=0 \text {. }
$$

En faisant dans la même formule $\alpha=1, \beta=B, \gamma=0$, on a:

$$
H_{0 B, \lambda \mu}+H_{1 B, \lambda \mu}=0
$$

et l'ensemble des relations (21-17), (21-18) est équivalent à (21.2). Par suite si $H_{\alpha \beta, \lambda \mu}$ et $l_{\alpha}$ isotrope sont déjà liés par les relations $(21.3)$ et $(21-8)$, les relations (21-2) ne se traduisent que par (21.17) soit

$$
H_{23, \lambda \mu}=0 \text {. }
$$


Nous nous proposons d'établir que pour $m=3$, (21.2) est une conséquence de $(21-3)$ et $(21.8)$ où $l_{x}$ est supposé isotrope. D'après $(21-16,) H_{23,10}=0$. De plus d'après cette même relation:

$$
H_{A 0, B 0}=-H_{A 1, B 0}=H_{A 1, B 1} \text {. }
$$

Pour faire intervenir (21-8) remarquons qu'en repère orthonormé, on a, compte-tenu de la signature

$$
H_{\alpha \beta}=H_{x, \beta 0}-H_{x u, \beta u} .
$$

De $(21-8)$ on déduit ainsi

$$
\left\{\begin{array}{l}
H_{A B}=H_{A 0, B 0}-H_{A 1, B 1}-H_{A C, B C}=0 \\
H_{1 B}=-H_{1 A, B A}=0 \\
H_{0 B}=-H_{0 A, B A}=0 .
\end{array}\right.
$$

En donnant dans $(21-19)$ a $A, B, C$ les valeurs 2 et 3 , on obtient:

$$
H_{23,23}=0 \quad H_{23,31}=H_{23,12} \quad H_{23,20}=H_{23,30}=0
$$

c'est-à-dire (21.17): (21.2) est donc ètabli. Nous énoncerons:

THĖoRÈmE. - Etant donnés, en un point $x$ d'une variété riemannienne $V_{4}$ admettant une métrique de type hyperbolique normal un tenseur $H_{x \beta, \lambda \mu}$ jouissant des propriétés de symétrie (21.1) et un vecteur $l^{x}$ isotrope, si ces élements satisfont (21-3) et (21-8), ils satisfont (21-2).

e) Le tenseur $H_{x, \beta, \gamma \mu}$ peut ètre identifié à un tenseur symétrique construit sur l'espace des bivecteurs en $x$. Cet espace admet la mètrique définie par le tenseur

$$
\gamma_{\alpha \beta ., \lambda_{\mu}}=g_{\alpha \lambda} g_{\beta \mu}-g_{\alpha, \mu} g_{\beta \lambda} .
$$

Dans le cas de la variété $V_{4}$ de la relativité générale; l'espace des bivecteurs envisagé est de dimension 6 . Si $\overrightarrow{e_{\lambda}}$ est un repère orthonormé en $x$ de $V_{4}$, nous convenons de poser:

$$
\left\{\begin{array} { l } 
{ \vec { e _ { 2 } } \wedge \vec { e _ { 3 } } = \tau _ { 1 } } \\
{ \vec { e _ { 3 } } \wedge \vec { e _ { 1 } } = \tau _ { 2 } } \\
{ \vec { e _ { 1 } } \wedge \vec { e _ { 2 } } = \tau _ { 3 } }
\end{array} \quad \left\{\begin{array}{l}
\overrightarrow{e_{1}} \wedge \overrightarrow{e_{0}}=\tau_{4} \\
\overrightarrow{e_{2}} \wedge \overrightarrow{e_{0}}=\tau_{5} \\
\overrightarrow{e_{3}} \wedge \overrightarrow{e_{0}}=\tau_{8} .
\end{array}\right.\right.
$$

Les $\tau_{j}(I, J,=1, \ldots 6)$ définissent une base de l'espace envisagé. orthonormée pour la métrique determinée par (21.20). Dans cette base, le tenseur métrique (21.20) admet pour seules composantes non nulles:

$$
\gamma_{11}=\gamma_{22}=\gamma_{33}=1 \quad \gamma_{44}=\gamma_{55}=\gamma_{66}=-1 .
$$

Ce qui nous fournit la signature de cette métrique. Nous nous proposons d'étudier la matrice représentative du tenseur $H_{\alpha \beta, \lambda_{2 .}{ }^{2}}$ dans la base ainsi introduite. C'est une matrice symétrique $6 \times 6$ que nous noterons $\left(H_{I J}\right)$. 
Pour $e_{2}=n^{(1)}, e_{3}=n^{(2)}$ les deux formes $\varphi^{(i)}$ ont pour composantes:

$$
\left\{\begin{array} { l l } 
{ \varphi _ { 2 3 } ^ { ( 1 ) } = 0 } & { \varphi _ { 1 0 } ^ { ( 1 ) } = 0 } \\
{ \varphi _ { 3 1 } ^ { ( 2 ) } = 0 } & { \varphi _ { 2 0 } ^ { ( 1 ) } = 1 } \\
{ \varphi _ { 1 2 } ^ { ( 1 ) } = 1 } & { \varphi _ { 3 0 } ^ { ( 1 ) } = 0 }
\end{array} \quad \left\{\begin{array}{lll}
\varphi_{23}^{(2)}= & 0 & \varphi_{10}^{(2)}=0 \\
\varphi_{31}^{(2)}=-1 & \varphi_{20}^{(2)}=0 \\
\varphi_{12}^{(2)}=0 & \varphi_{30}^{(2)}=1
\end{array}\right.\right.
$$

De (21.9), il résulte pour la matrice $\left(H_{I J}\right)$ la forme:

$$
\left(H_{l J}\right)=\left(\begin{array}{cccccc}
0 & 0 & 0 & 0 & 0 & 0 \\
0 & a_{22} & -a_{12} & 0 & -a_{12} & -a_{22} \\
0 & -a_{12} & a_{12} & 0 & a_{11} & a_{12} \\
0 & 0 & 0 & 0 & 0 & 0 \\
0 & -a_{12} & a_{11} & 0 & a_{11} & a_{12} \\
0 & -a_{22} & a_{12} & 0 & a_{12} & a_{22}
\end{array}\right)
$$

$H_{\alpha \beta}$ satısfait (21.8) avec

$$
\tau=-\left(a_{11}+a_{22}\right)
$$

\section{2. - Matrice des discontinuités du tenseur de curbure en relativité générale.}

a) En point $x$ d'une hypersurface $S$ à la traversèe de laquelle le tenseur de courbure $R_{\alpha \beta, \lambda \mu}$ de la variété espace-temps $V_{4}$ est discontinu, étudions la matrice $\left(\left[R_{I J}\right]\right)(I, J=1, \ldots, 6)$ représentative du tenseur $\left[R_{\alpha \beta, 2, \mu}\right]$ relativement à un repère orthonormé tel que $\vec{l}=\overrightarrow{e_{0}}+\overrightarrow{e_{1}}$.

Des résultats du paragraphe précédent, il est aisé de déduire sa forme puisque $\left[R_{\alpha \beta, \lambda \mu}\right]$ satisfait aux relations $(21-2)$ et (21.3). Le tenseur contracté $\left[R_{\alpha \beta}\right]$ est ici supposé nul; il résulte alors de (21.23) que:

Posons:

$$
a_{11}+a_{12}=0 \text {. }
$$

$$
a_{11}=-a_{22}=\sigma \quad a_{11}=\rho .
$$

On obtient ainsi pour la matrice ([$\left.R_{I J}\right]$ ) la forme $\left({ }^{6}\right)$ :

$$
\left(\left[R_{I J}\right]\right)=\left(\begin{array}{rrrrrr}
0 & 0 & 0 & 0 & 0 & 0 \\
0 & -\sigma & -\rho & 0 & -\rho & \sigma \\
0 & -\rho & \sigma & 0 & \sigma & \rho \\
0 & 0 & 0 & 0 & 0 & 0 \\
0 & -\rho & \sigma & 0 & \sigma & \rho \\
0 & \sigma & \rho & 0 & \rho & -\sigma
\end{array}\right)
$$

(5) Cette forme a été obtenue par PrRani [2] en utilisant les coordonnées locales intro. duites au $\$ 8$. 
Le tenseur $\left[R_{\alpha \beta}, \lambda, u\right]$ étant donné, on peut naturellement par un choix convenable des vecteurs $\overrightarrow{e_{2}}$ et $\overrightarrow{e_{3}}$ faire $\rho=0$. Selon $(21.9)$, (22-1) peut etre traduite par:

$$
\left[R_{I J}\right]=\sigma\left(\varphi_{I}^{(1)} \varphi_{J}^{(1)}-\varphi_{I}^{(1)} \varphi_{J}^{(2)}\right)+\rho\left(\varphi_{I}^{(1)} \varphi_{J}^{(1)}+\varphi_{J}^{(1)} \varphi_{I}^{(2)}\right) .
$$

b) Nous avons vu (voir (17-8)) que:

$$
\left[R_{\alpha \beta, \lambda \mu}\right]=\frac{1}{2}\left(a_{\alpha \mu} l_{\beta} l_{\lambda}+a_{\beta \lambda} l_{\alpha} l_{\mu}-a_{\alpha \lambda} l_{\beta} l_{\mu}-a_{\beta \mu} l_{\alpha} l_{\lambda}\right)
$$

où les $a_{\alpha \lambda}$ ne sont astreints qu'à la condition (18.12) soit:

$$
a_{\alpha \lambda} l^{\lambda}=\frac{\alpha}{2} l_{\alpha}
$$

et sont définis à la transformation près:

$$
a_{\alpha \lambda} \rightarrow a_{\alpha \lambda}+t_{\alpha} l_{\lambda}+t_{\lambda} l_{\alpha}
$$

$a$ se trasforme selon:

$$
a \rightarrow a+2 t_{\alpha} l^{\alpha}
$$

et il est possible de choisir $t_{\alpha}$ de façon à annuler $a$. Supposons qu'il en soit ainsi; pour ces $a_{\alpha \lambda}$ restreints, la transformation (22.5) est astreinte à la condition $t_{\alpha} l^{\alpha}=0$; si nous posons alors.

$$
b_{\alpha \curlywedge}=-\frac{1}{2} a_{\alpha \lambda}
$$

(22-3) prend la forme (21.12) et d'après (22.4) les $b_{\alpha \lambda}$ satisfont bien (21-13). Il en résulte en particulier que tout tenseur au point $x$ de $V_{4}$ satisfaisant aux propriétés de symétrie (21-1) et aux relations (21-2) et (21-3) peut être le tenseur de discontinuité en $x$ du tensenr de courbure à la traverșée du 3-plan tangent au cone elémentaire en $x$ le long de $\vec{l}$.

On vérifie immédiatement que le scalaire ( $\left.{ }^{7}\right)$ :

$$
a_{\alpha \lambda} a^{\alpha \lambda}-\frac{1}{2} a^{2}
$$

est invariant par la transformation générale (22.5). Par suite on a pour le tenseur $\left[R_{\alpha \beta, \lambda \mu}\right]$

$$
e=\frac{1}{4}\left(a_{\alpha \lambda} a^{\alpha \lambda}-\frac{1}{2} a^{2}\right)=b_{\alpha \lambda} b^{\alpha \lambda}>0
$$

(7) L'introduction de cette quantité est due à Stellmadher. 
A. LICHNERowrCz: Ondes et radiations électromagnétiques, etc.

23. - Relations différentielles sur les discontinuités du tenseur de courbare.

Revenons à une variété $V_{m+1}$ munie d'une métrique de type hyperbolique normal satisfaisant ̀̀ des équation d'Einstein à second membre nul. Si la courbure de $V_{m+1}$ est discontinue à la traversée d' une hypersurface $S$, le tenseur $\left[R_{\alpha \beta}, \lambda_{\mu}\right]$ satisfait sur $S \grave{a}$ une relation différentielle intéressante analogue à (9-11).

Soit $f\left(x^{\alpha}\right)=0$ l'équation locale de $S$ où $f$ est supposé de classe $C^{2}$. Pour $l_{\alpha}=\partial_{\alpha} f$, on a

$$
\nabla_{\alpha} l_{\beta}-\nabla_{\beta} l_{\alpha}=0
$$

a) Sur un voisinage $U$ adoptons des coordonnées locales et des notations idendiques à celles du $\S 9$. En particulier dans ces coordonnées $\nabla_{0} l^{\circ}=0$. Le tenseur $\left[R_{\alpha \beta, \lambda_{\mu}}\right]$ satisfait sur $S$ aux relations:

$$
l_{\rho}\left[R_{\alpha \beta, \lambda \mu}\right]+l_{\lambda}\left[R_{\alpha \beta, \mu \rho}\right]+l_{\mu}\left[R_{\alpha \beta, \rho \lambda}\right]=0
$$

et

$$
l_{\rho}\left[R_{\alpha \beta}, \rho_{\mu}\right]=0 .
$$

Dans nos coordonnées locales, (23-2) et (23-3) s'écrivent respectivement:

$$
\begin{aligned}
& {\left[R_{\alpha \beta, u v}\right]=0} \\
& {\left[R_{\alpha \beta},{ }^{0}\right]=0 .}
\end{aligned}
$$

b) La relation (23-2) étant satisfaite sur $S$, il vient en la dérivant sur cette hypersuface:

$$
\nabla_{u}\left(l_{\rho}\left[R_{\alpha \beta, \lambda \mu}\right]\right)+\nabla_{u}\left(l_{\lambda}\left[R_{\alpha \beta, \mu}{ }^{p}\right]\right)+\nabla_{u}\left(l_{\mu}\left[R_{\alpha \beta},{ }^{\circ}\right]\right)=0
$$

En donnent à $\rho$ la valeur $u$ et sommant, on obtient une relation qui, compte tenu de $\nabla_{0} l^{0}=0$, peut s'écrire:

$$
l \rho \nabla_{\rho}\left[R_{\alpha \beta, \lambda \mu}\right]+\left(\nabla_{\rho} l \rho\right)\left[R_{\alpha \beta, \lambda \mu}\right]+Q_{\alpha \beta, \lambda \mu}=0
$$

où l'on a posé:

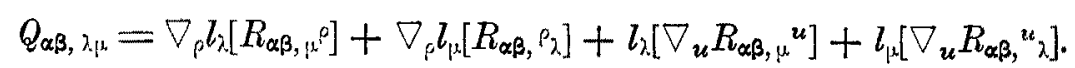

Comme $R_{\alpha \beta}=0$, on sait que:

$$
\nabla_{p} R_{\alpha \beta, p^{p}}=0
$$

Par suite $Q_{\alpha \beta, \lambda_{j} \mu}$ peut se mettre sous la forme:

$$
Q_{\alpha \beta, \lambda \mu}=\left[\nabla_{\lambda} l_{\rho} R_{\alpha \beta, \mu}{ }^{p}+\nabla_{\mu} l_{\rho} R_{\alpha \beta,}{ }^{\rho_{\lambda}}\right]-l_{\lambda}\left[\nabla_{0} R_{\alpha \beta, \mu}{ }^{0}\right]-l_{\mu}\left[\nabla_{0} R_{\alpha \beta,}{ }^{0} \lambda\right] .
$$


Or:

$$
\nabla_{\lambda} l_{\rho} \cdot R_{\alpha \beta, \mu}{ }^{\rho}+\nabla_{\mu} l_{\rho} \cdot R_{\alpha, \beta},{ }^{\rho_{\lambda}}=\nabla_{\lambda}\left(l_{\rho} R_{\alpha \beta, \mu}{ }^{\rho}\right)+\nabla_{\mu}\left(l_{\rho} R_{\alpha \beta,},{ }_{\lambda}\right)-l_{\rho}\left(\nabla_{\lambda} R_{\alpha \beta, \mu}{ }^{\rho}+R_{\alpha \beta},{ }_{\lambda}\right) .
$$

De l'identité de Branchr on déduit ainsi :

$$
Q_{\alpha \beta, \lambda \mu}=l \rho \nabla_{\rho}\left[R_{\alpha \beta, \lambda \mu}\right]+\left[\nabla_{\lambda}\left(l_{\rho} R_{\alpha \beta, \mu}{ }^{P}\right)+\nabla_{\mu}\left(l_{\rho} R_{\alpha \beta,}, \beta_{\lambda}\right)\right]-l_{\lambda}\left[\nabla_{0} R_{\alpha \beta, \mu}{ }^{0}\right]-l_{\mu}\left[\nabla_{0} R_{\alpha \beta,}, \lambda\right] .
$$

En faisant $\lambda=u$ dans le second terme du second membre, on a:

$$
\left[\nabla_{u}\left(l_{\rho} R_{\alpha \beta, \mu} \rho\right)\right]=\nabla_{u}\left(l_{\rho}\left[R_{\alpha \beta, \mu^{?}}\right]\right)=0
$$

Par suite:

$$
Q_{\alpha \beta, u v}=l p \nabla_{\rho}\left[R_{\alpha \beta, u v}\right]
$$

De même $\lambda=0, \mu=u$

$$
\left[\nabla_{0}\left(l_{\rho} R_{\alpha \beta, u^{\rho}}\right)\right]=\left[\nabla_{0} l \rho R_{\alpha \beta, u_{\rho}}+l_{\rho} \nabla_{0} R_{\alpha \beta, u^{p}}\right]=\left[\nabla_{0} R_{\alpha \beta, u^{0}}\right]
$$

puisque $\nabla_{0} l^{\circ}=0$. On obtient ainsi:

$$
Q_{\alpha \beta . \lambda \mu}=l p \nabla_{\phi}\left[R_{\alpha \beta, \lambda \mu}\right]
$$

En reportant dans (23-6), on voit que le tenseur $\left[R_{\alpha \beta,}, \mu\right]$ satisfait sur $S$ à la relation différentielle $\left(^{s}\right)$ :

$$
2 l p \nabla_{p}\left[R_{\alpha \beta}, \lambda_{\beta}\right]+(\nabla \ell l)\left[R_{\alpha \beta, \lambda_{\mu}}\right]=0
$$

qui entraìne des conséquences analogues à (9-11) en ce qui concerne $\left[R_{\alpha \beta}, \lambda_{[\alpha}\right]$ : si le tenseur $\left[R_{\alpha \beta, \lambda \mid}\right]$ s'annule en un point $x$ de $S$, il s'annule tout le tong de la géodésique isotrope issue de $x$ et située sur $S$.

Soit $\Sigma$ une hypersurface orientée dans l'espace et coupant $S\left(x^{0}=0\right)$ selon une 2-surface $U$. Donnons-nous sur $\Sigma$ des données de CAUCHY $\left(g_{\alpha \beta}\right)_{\Sigma},\left(\partial 2 g_{\alpha \beta}\right)_{\Sigma}$, telles qu'à la traversée de $S$ les dérivées secondes admettent les discontinuités $\left[\partial_{00} g_{\alpha \beta}\right]_{4}=\left(a_{\alpha \beta}\right)_{4}$, les $\left(a_{\alpha \beta}\right)_{U}$ n'étant astreints qu'à la condition (18.12)

$$
\left\{\left(a_{\alpha \beta}-\frac{a}{2} g_{\alpha \beta}\right) l^{\beta}\right\}_{U}=0 \text {. }
$$

Cela revient à se donner aux points de $U$ un tenreur $\left[R_{\left.\alpha \beta, \lambda_{\mu}\right]}\right]_{U}$ admettant $(l P)_{U}$ comme vecteur fondamental et dont le tenseur contracté soit nul. Aux donnés de CAUchx envisagées correspond une solution des équations d' Ensstein $R_{\alpha \beta}=0$ dont le tensenr de courbure admet à la traversée de $S$ une discontinuité $\left[R_{\alpha \beta, \gamma_{i}}\right]$. Le tenseur $\left[R_{\alpha \beta, \lambda_{1}, \mu}\right]$ est nécessairement la solution de (23-8) correspondant aux données initiales $\left[R_{\alpha \beta}, \lambda_{i p}\right] U$.

(8) Cette relation a été établie indépendammente par A. Trautman [1]. 
A. Licinerowicz; Ondes et radiations électromagnétiques, etc.

c) Nous arons vu que le tenseur $\left[R_{\alpha \beta, \lambda}\right]$ peut s'ecrire:

$$
\left[R_{\alpha \beta, \lambda_{\mu}}\right]=b_{\alpha \lambda} l_{\beta} l_{\mu}+b_{\beta,} l_{\alpha} l_{\lambda}-b_{\beta \mu} l_{\beta} l_{\lambda}-b_{\beta \lambda} l_{\alpha} l_{\mu}
$$

où les $b_{\alpha \varkappa}$ satisfont à :

$$
b_{\alpha \lambda} l^{\lambda}=0 \text {. }
$$

Posons:

$$
c_{\alpha \lambda}=2 l_{p} \nabla_{p} b_{\alpha \lambda}+(\nabla p l p) b_{\alpha \lambda} .
$$

En tenant compte de $l_{p} \nabla_{\rho} l_{\alpha}=0$, on voit que (23.8) peut s'écrire:

$$
c_{\alpha \lambda} l_{\beta} l_{\mu_{\mu}}+c_{\beta \mu} l_{\alpha} l_{\lambda}-c_{\alpha \mu} l_{\beta} l_{\lambda}-c_{\beta \lambda} l_{\alpha} l_{\mu}=0
$$

Par produit de (23-9) par $b^{\alpha \lambda}$ il vient:

$$
b^{\alpha \lambda} c_{\alpha \lambda}=0
$$

soit

$$
2 l \rho b \alpha \beta \nabla_{\rho} b_{\alpha \beta}+\left(\nabla_{\rho} l \rho\right) b^{\alpha \beta} b_{\alpha \beta}=0
$$

Ainsi le scalaire

$$
e=b^{\alpha \beta} b_{\alpha \beta}=\frac{1}{4}\left(a^{\alpha \beta} a_{\alpha \beta}-\frac{1}{2} a^{2}\right)>0
$$

satisfait à l'identité de conservation:

$$
\nabla_{p}(e l \rho)=0
$$

relation semblable à (10-1). Introduisons le tenseur d'ordre 4:

$$
\tau_{\alpha \beta, \lambda \mu}=e l_{\alpha} l_{\beta} l_{\lambda} l_{\mu *}
$$

Si $\vec{l}$ subit la transformation $l_{\alpha} \rightarrow \lambda l_{\alpha}$, alors $b_{\alpha \lambda} \rightarrow \lambda^{-} b_{\alpha, \text {, et }} e \rightarrow \lambda^{-4} e$. Le tenseur $\tau$ ne dépend donc que du tenseur $\left[R_{\alpha \beta, \lambda, k}\right]$ et, compte-tenu de $l_{a} \nabla_{\rho} l_{\alpha}=0$, (23.11) exprime que $\tau$ est conservatif:

$$
\nabla_{\alpha} \tau_{\beta \lambda_{\mu} \mu^{\alpha}}=0 \text {. }
$$

On vérifie d'ailleurs immédiatement sur l'expression de $\left[R_{\alpha \beta, \lambda_{\mu}}\right]$ en termes des $b_{\alpha \lambda}$ que:

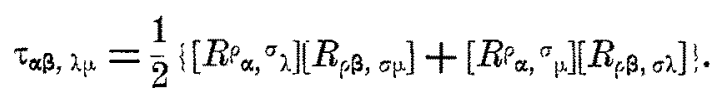

\section{3 bis. - Cas ot il existe un champ électromagnétique.}

Dans le cas où il existe dans $V_{4}$ un ehamp électromagnétique satisfaisant aux équations de Maxwall du vide et relié au champ gravitationnel 
par les équations d' EInstein

$$
R_{\alpha \beta}=\chi \tau_{\alpha \beta} \quad\left(\tau_{\alpha \beta} \text { tenseur de MAXwELL }\right)
$$

supposons qu'à la traversée de l'hypersuface $S$ le tenseur dérivé du champ électromagnétique et le tenseur de courbure soient discontinus.

a) Les hypotèses et les notations étant identiques à celles du $\S 9$, la formule (9.11) doit etre modifié: l'identité de Ricci donne ici

$$
\left[\nabla_{\rho} \nabla_{\sigma} F_{\beta \gamma}-\nabla_{\sigma} \nabla_{\rho} F_{\beta \gamma}\right]=-\left[R_{\beta, \rho \sigma}^{\lambda}\right] F_{\lambda \gamma}-\left[R_{\gamma, \rho \sigma}^{\lambda}\right] F_{\beta \lambda} .
$$

Il en résulte aisément:

$$
\left[l_{\beta} \nabla_{\rho} \nabla_{\sigma} F_{\gamma^{\rho}}+l_{\zeta} \nabla_{\rho} \nabla_{\sigma} F_{\rho_{\beta}}\right]=-l_{\lambda} F^{\lambda{ }^{\lambda}}\left[R_{\rho \sigma, \beta_{Y}}\right]
$$

et

$$
\left[l_{\rho} \nabla_{\beta} \nabla_{\sigma} F_{\gamma}{ }^{\rho}+l_{\rho} \nabla_{\gamma} \nabla_{\sigma} F_{\rho_{\beta}}\right]=-l_{\rho} \nabla_{\rho}\left[V_{\sigma} F_{\beta \gamma}\right]+l_{\lambda} F^{\lambda \rho}\left[R_{\rho \sigma, \beta_{\gamma}}\right] .
$$

On en déduit par le raisonnement même du $\S 9$ :

(23 bis-2)

$$
2 l_{\rho} \nabla_{\rho}\left[\nabla_{\sigma} F_{\beta_{\gamma}}\right]+\left(\nabla_{\rho} l \rho\right)\left[\nabla_{\sigma} F_{\beta_{\gamma}}\right]=2 l_{\lambda} F^{\lambda \rho}\left[R_{\rho \sigma}, \beta_{Y}\right] .
$$

En posant dans (23 bis-2):

(23 bis.3

$$
\left[\nabla_{\sigma} F_{\beta_{\gamma}}\right]=\varphi_{\beta_{\gamma}} l_{\sigma}=\left(l_{\beta} b_{\gamma}-l_{\gamma} b_{\beta}\right) l_{\sigma}
$$

et

(23 bis.4) $\quad\left[R_{\rho \sigma, \beta \gamma}\right]=b_{\beta \beta} l_{\sigma} \gamma_{Y}+b_{\sigma \gamma} l_{\beta} l_{\beta}-b_{\rho \gamma} l_{\sigma} l_{\beta}-b_{\sigma \beta} l_{\rho} l_{\varphi} \quad\left(b_{\beta}^{\beta}=0\right)$

il vient

$\left(23\right.$ bis-5) $\quad 2 l_{\rho} \nabla_{\rho}\left(l_{\beta} b_{\gamma}-l_{\gamma} b_{\beta}\right)+\left(\nabla_{\rho} l_{\rho}\right)\left(l_{\beta} b_{\gamma}-l_{\gamma} b_{\beta}\right)=2 l_{\lambda} F^{\lambda} \lambda_{\rho}\left(b_{\beta} l_{\gamma}-b_{\rho \gamma} l_{\beta}\right)$.

\section{Posons}

(23 bis-6)

$$
e_{e m}=-b^{\beta} b_{\beta}>0 \quad e_{g}=b^{\alpha \lambda} b_{\alpha \lambda}>0 .
$$

En multipliant ( 23 bis -5 ) par $b^{\beta}$ il vient:

$$
\nabla_{\rho}\left(e_{e m} l \rho\right)=2 l_{\lambda} F^{\lambda \rho} b_{\sigma} \sigma b_{\rho \sigma} .
$$

b) Reprenons maintenant la raisonnement du $\$ 23$ en notant qu'au lieu de $R_{\alpha \beta}=0$ on a ici (23 bis-1). Il vient:

$$
\left[\nabla R_{\alpha \beta, \mu}{ }^{\rho}\right]=-\chi\left[\nabla_{\alpha} \tau_{\beta \mu}-\nabla_{\beta} \tau_{\alpha \mu \mu}\right] .
$$


Par suite à la formule (23.8) se trouve substituée la formule:

(23 bis-8) $2 l_{\circ} \nabla_{\rho}\left[R_{\left.\alpha \beta, \lambda_{\mu}\right]}\right]+\left(\nabla_{\rho} l \rho\right)\left[R_{\alpha \beta, \lambda \mu}\right]=\chi l_{\lambda}\left[\nabla_{\alpha} \tau_{\beta, \mu}-\nabla_{\beta} \tau_{\alpha i k}\right]-\chi l_{\mu}\left[\nabla_{\alpha} \tau_{\beta \lambda}-\nabla_{\beta} \tau_{\alpha \lambda}\right]$.

Or:

$$
\left[\nabla_{\beta} \tau_{\alpha \lambda}\right]=\frac{1}{2} g_{\alpha \lambda} F^{\rho \sigma}\left[\nabla_{\beta} F_{p, \sigma}\right]-F_{\lambda}\left[\nabla_{\beta} F_{\alpha, \rho}\right]-F_{\alpha \rho}\left[\nabla_{\beta} F_{\lambda} \rho\right]
$$

soit :

(23 bis.9) $\quad\left[\nabla_{\beta} \tau_{\alpha \lambda}\right]=\frac{1}{2} g_{\alpha \lambda} F^{\rho \sigma} \varphi_{\beta \sigma} l_{\beta}-F_{\lambda} \rho^{p} \varphi_{\alpha_{\rho}} l_{\alpha}-F_{\alpha_{\beta}} \varphi_{\lambda} \rho l_{\beta}$.

En substituant dans (23 bis-8) les expressions de $\left[R_{\alpha \beta}, \lambda_{\mu}\right]$ et $\left[\nabla_{\beta} \tau_{\alpha \lambda \lambda}\right]$ et en multipliant par $b^{\alpha \lambda}$, il vient:

(23 bis-10)

$$
\nabla_{\rho}\left(e_{g} l_{\rho}\right)=-2 \chi l_{\lambda} F^{\lambda \rho} b^{r} b_{\rho \sigma} .
$$

De (23 bis-7) et (23 bis-10) on tire:

$$
\nabla_{p}\left\{\left(e_{g}+e_{e m}\right) l_{\rho}\right\}=0
$$

qui traduit la conservation de «l'énergie de discontinuite » totale relative au champ gravitationnel et au champ électromagnétique. Le tenseur d'ordre 4 obtenu en combinant (23.14) et (10-2) est donc conservatif.

\section{Radiation gravitationnelle en relativitẻ générale.}

\section{Notion de radiation gravitationnelle pure.}

a) De l'étude fait dans la partie II, il résulte que l'on doit s'intéresser en relativité générale aux métriques pour lesquelles il existe un vecteur $l_{\alpha}$ tel que le tenseur de courbure $R_{\alpha \beta, \lambda \mu}$ satisfasse aux relations

$$
l_{\alpha} R_{\beta, \lambda \mu}+l_{\beta} R_{\gamma \alpha, \lambda \mu}+l_{\gamma} R_{\alpha \beta, \lambda \mu}=0
$$

et

$$
l^{\alpha} R_{\alpha \beta . \lambda \mu}=0 .
$$

Si $R_{\alpha \beta, \lambda \mu}$ n'est pas identiquement nul $l_{\alpha}$ est nécessairement isotrope. The tenseur de Riccr de la métrique est alors de la forme

$$
R_{\alpha \beta}=\tau l_{\alpha} l_{\beta} .
$$

S'il en est ainsi en un point $x$ de la variété $V_{4}$, nous dirons $q u$ ' en ce point la métrique correspond à un état de radiation totale pure. Si en outre le tensenr de RICCI $R_{\alpha \beta}$ est nul, nons dirons que nous avons on état de radiation gravitationnelle pure. 
Nous montrerons ultérieurement sur un exemple $q u$ 'il peut effectivement en ètre ainsi en tous les points d'un domaine quadrimensionnel de la variété $V_{4}$. Sur un tel domaine $l_{\alpha}$ définit un champ de génératrices de cônes élémen. téires et par. suite un chnmp de 3-plans tangents aux cônes le long de ces génératrices. Si ce champ de plans est complètement intégrable, nous dirons que la radiation envisagée est de type intégrable.

b) Sur la varieté $V_{m+1}$ supposons qu'il existe un champ de vecteurs $l_{\alpha}$ tel que (24-1) et (24.2) soient satisfaites. Do l'identité de BranchI:

$$
\nabla_{\rho} R_{\alpha \beta, \gamma_{\mu}}+\nabla_{\alpha} R_{\beta_{1}, \lambda_{\mu}}+\nabla_{\beta} R_{\rho \alpha, \lambda \mu}=0
$$

on dédnit par produit contracté par lf, compte-tenu de (24-2):

$$
l^{\rho} \nabla_{\rho} R_{\alpha \beta, \lambda \mu}-\nabla_{\alpha} l_{\rho} R_{\beta}{ }^{p} \lambda_{\mu}-\nabla_{\beta} l_{\rho} R_{\alpha_{s} \lambda_{\mu}}=0
$$

cette relation joue ici le même rôle que (11-8) on $L \overrightarrow{(l)} F=0$ dans le cas des radiations électromagnétiques.

Supposons $R_{\times \beta}=0$. Par dérivation contractée de:

$$
l_{\rho} R_{\alpha \beta, \lambda \mu}+l_{\alpha} R_{\beta_{\rho}, \lambda \mu}+l_{\beta} R_{q, \alpha, \lambda_{\mu}}=0
$$

il vient, compte tenu de $\nabla_{\rho} R_{\beta^{0} . \lambda_{1}}=0$

$$
\nabla_{\rho}\left(l_{l} R_{\alpha \beta, \lambda_{\mu}}\right)+\nabla_{\rho} l_{\alpha} R_{\beta^{2}, \lambda_{i}}+\nabla_{\rho} l_{\beta} R_{\alpha, \lambda_{\mu}}^{p}=0
$$

qui jone ici le rôle de (11-9).

Supposons en outre le champ de type intégrable $\nabla_{\alpha} l_{\rho}=\nabla_{p} l_{\alpha}$ et par addition de (24-4) et (24-5), on obtient la relation :

$$
2 l_{i} \nabla \rho R_{\alpha \beta, \beta, \mu^{\alpha}}+\left(\nabla \rho l^{\circ}\right) R_{\alpha \beta, \lambda_{j, \mu}}=0
$$

qui est semblable $\dot{a}(14 \cdot 4)$.

c) Si le tenseur de Ricc $R_{\alpha \beta}$ de $V_{m+1}$ est $\neq 0$, il est clair que les trajectoires du champ de vecteur défini par $l_{\alpha}$ sont des géodésiques isotropes. En effet on a nécessairement $R=0$ et par suite

$$
S_{\alpha \beta}=\tau l_{\alpha} l_{\beta} \quad(\tau \neq 0) .
$$

Des identités de conservation, on déduit:

$$
\nabla_{\alpha} S_{\beta}^{\alpha}=\nabla_{\alpha}\left(\tau l^{\alpha}\right) l_{\beta}+\tau l^{\alpha} \nabla_{\alpha} l_{\beta}=0 .
$$

Ainsi pour $\tau \neq-0, l^{\alpha} \nabla_{\alpha} l_{\beta}$ est bien colicéaire à $l_{\beta}$.

Il est aisé d'étendre ce résultat au cas général. Nous utiliserons à cet effet (24.4) et introduisons le tenseur défini par

$$
P_{\alpha \beta, \lambda_{j}}=l_{i} \nabla_{\rho} R_{\alpha \beta, Z_{j k}}
$$


qui présente le même type de symétrie que le tenseur de courbure. De (24-4) on dédnit que le tenseur $P_{\alpha \beta, \lambda \mu}$ satisfait aux relations:

$$
l_{\nu} P_{\alpha \beta, \lambda \mu}+l_{\lambda} P_{\alpha \beta, \mu \nu}+l_{\mu} P_{\alpha \beta, \nu \lambda}=0
$$

et

$$
l^{\lambda} P_{\alpha \beta, \lambda \mu}=0 .
$$

D'autre part dérivons (24-1) et effectuons le produit contracté par $l_{\rho}$. Si nous posons $l \rho \nabla l_{\alpha}=u_{\alpha}$, il vient:

$$
u_{\alpha} R_{\beta \gamma, \lambda_{\mu}}+u_{\beta} R_{\gamma \alpha, \lambda_{\mu}}+u_{\gamma} R_{\alpha \beta, \lambda \mu}+l_{\alpha} P_{\beta \gamma, \lambda_{\mu}}+l_{\beta} P_{\gamma \alpha, \lambda_{\beta}}+l_{\gamma} P_{\alpha \beta, \lambda \mu}=0 .
$$

En procédant de même sur (24-2) on a :

$$
u^{\alpha} R_{\alpha \beta, \lambda \mu}+l^{\alpha} P_{\alpha \beta, \lambda \mu}=0 .
$$

De (24.8) et (24.9) on déduit ainsi que le vecteur $u_{\alpha}$ et le tenseur de courbure satisfont aussi aux relations (24-1) et (24-2). Le tenseur de courbure n'étant par identiquement nul, $u_{\alpha}$ est nécessairement isotrope: mais il est orthogonal à $l_{\alpha}$. Il ne peat done que lui être colinéaire. Ainsi :

Théorkme. - Si, sur une variété riemannienne $V_{m+1}$ dont la métrique de type hyperbolique normal est à tenseur de courbure non nul, il existe un champ de vecteurs $l_{\alpha}$ satisfaisant aux relations (24-1) et 24-2) les trajectoires de ce champ de vecteurs sont des géodésiques de longueur nulle de la métrique.

\section{L'identité de conservation.}

Nous nous proposons d'établir pour une radiation gravitationnelle pure une identité de conservation analogue à (11-11). A cet effet nous adapterons au cas gravitationnel la méthode indiquée au $\S 11 \mathrm{~d}$.

En vertu de (24-1) et (24-2) nous pouvons poser:

$$
R_{\alpha \beta, \lambda \mu}=b_{\alpha, 2} l_{\beta} l_{\mu}+b_{\beta \mu} l_{\alpha} l_{\lambda}-b_{\alpha \mu} l_{\beta} l_{\lambda}-b_{\beta \lambda} l_{\alpha \alpha} l_{\mu}
$$

où les $b_{\alpha \lambda}$ satisfont à :

$$
b_{\alpha 2} l^{\lambda}=0
$$

et où $l$ est astreint à

$$
l p \nabla_{p} l_{\alpha}=0
$$

Posons $d_{\alpha \lambda}=l \nabla_{\rho} b_{\alpha \lambda}$. En substituant dans (24-4) l'expression précédente de $R_{\alpha \beta, \lambda \mu}$, il vient :

$$
\begin{gathered}
d_{\alpha \lambda} l_{\beta} l_{\mu}+d_{\beta \mu} l_{\alpha} l_{\lambda}-d_{\alpha_{\mu}} l_{\beta} l_{\lambda}-d_{\beta \lambda} l_{\alpha} l_{\mu}-\nabla_{\alpha} l_{\rho}\left(b_{\mu}{ }_{\mu} l_{\beta} l_{\lambda}-b_{\lambda_{\lambda}} l_{\beta} l_{\mu}\right)- \\
\nabla_{\beta} l_{\rho}\left(b_{\rho_{2}} l_{\alpha} l_{\mu}-b \rho_{\mu} l_{\alpha} l_{\lambda}\right)=0,
\end{gathered}
$$


Par multplication par $b^{\alpha \lambda}$, on obtient la relation:

$$
l \rho b^{\alpha \beta} \nabla_{\rho} b_{\alpha \beta}+\nabla_{\sigma} l_{\rho} b \rho_{\lambda} b^{\alpha \lambda}=0 .
$$

En substituant de même dans (24-5) l'expression de $R_{\alpha \beta, \lambda \mu}$ on a:

$$
\begin{aligned}
& \left(d_{\alpha \lambda}+(\nabla \rho p) b_{\alpha \lambda}\right) l_{\beta} l_{\mu}+\left(d_{\beta \mu}+\left(\nabla_{\rho} l_{\rho}\right) b_{\beta \mu}\right) l_{\mu} l_{\lambda}-\left(d_{\alpha \mu}+\left(\nabla_{\beta} l \rho\right) b_{\alpha \mu}\right) l_{\beta} l_{\lambda}-\left(d \beta \lambda+\left(\nabla_{\rho} l \rho\right) b_{\beta \lambda}\right) l_{\alpha} l_{\mu}+ \\
& +\nabla_{\beta} l_{\alpha}\left(b_{\beta} \rho_{\beta} l_{\lambda}-b \rho_{\lambda} l_{\beta} l_{\mu}\right)+\nabla_{\beta} l_{\beta}\left(b e_{\lambda} l_{\alpha} l^{\mu}-b p_{\mu} l_{\alpha} l_{\lambda}\right)=0 \text {. }
\end{aligned}
$$

Par multiplication par $b^{\alpha \lambda}$ il vient:

$$
l \rho b^{\alpha \beta} \nabla_{\rho} b_{\alpha \beta}+\left(\nabla_{\rho} l \rho\right) b^{\alpha \beta} b_{\alpha \beta}-\nabla_{\rho} l_{\sigma} b_{\lambda} b^{\alpha \lambda}=0 .
$$

En ajoutant membre a membre (25-1) et (25-2) on a :

$$
2 l \rho b^{\alpha \beta} \nabla_{p} b_{\alpha \beta}+\left(\nabla_{p} l \rho\right) b^{\alpha \beta} b_{\alpha \beta}=0
$$

soit

$$
\nabla_{p}(e l p)=0 \quad \text { arec } e=b^{\alpha \beta} b_{\alpha \beta}>0 .
$$

En introduisant le tenseur d'ordre 4

$$
\tau_{\alpha \beta \lambda \mu}=e l_{\alpha} l_{\beta} l_{\lambda} l_{\mu}
$$

qui ne dépend que du tenseur de courbure, on voit que, compte-tenu de (25-1), (25-4) exprime que $\tau$ est conservatif.

\section{Le tenseur de Bel.}

10) Au point $x$ de la variété riemanuienne $V_{4}$, considérons un tenseur $K_{\alpha \beta, y \delta}$ satisfaisant aux propriétés de symétrie (21.1) du tenseur de coubure et tel que son tenseur contracté vérifie:

$$
K_{\alpha \beta}=\lambda g_{\alpha, \beta} .
$$

Associons-lui le tenseur :

$$
K_{\alpha \beta, \gamma \delta}^{*}=\frac{1}{2} \eta_{\alpha \beta, \sigma \sigma} K_{, \gamma \delta}^{\rho \sigma}
$$

Il est aisé de montrer que, sous l'hypothèse (26.1), le tenseur (26-2) jouit encore des propriétés de symétrie (21.1) c'est-à-dire satisfait à ;

$$
K_{\alpha \beta, \gamma \delta}^{*}=K_{r \delta, \alpha \beta}^{*} \text { : }
$$

En effet si $S$ désigne la sommation après permutation circulaire sur les trois indice $\alpha, \beta, \gamma$ supposés distincts, il vient:

$$
S K_{\alpha \beta, \gamma^{\delta}}^{*}=\frac{1}{2} S \eta_{\alpha \beta_{\zeta} \sigma} K_{\gamma^{\sigma}, \gamma_{\delta}^{\delta}}=\eta_{\alpha \beta \gamma \sigma} K_{\delta \delta}^{\sigma} .
$$


On roit ainsi que, quels que soient $\alpha, \beta, \gamma, \delta$ on a:

$$
K_{\alpha \beta, \gamma \delta}^{*}+K_{\beta \gamma, \alpha \delta}^{*}+K_{\gamma \alpha, \beta \delta}^{*}=\lambda \eta_{\alpha \beta \gamma \delta}
$$

(26.4) peut aussi s' écrire:

$$
K_{\beta \alpha, \delta \gamma}^{*}+K_{\alpha_{\delta, \beta \gamma}}^{*}+K_{\delta \beta * \alpha \gamma}^{*}=\lambda \eta_{\alpha \beta \gamma \delta} .
$$

Par addition membre à membre on obtient:

$$
K_{\alpha \beta, \gamma \delta}^{*}=L_{\alpha \gamma, \beta \delta}+L_{\alpha \delta, \gamma \beta}+\lambda \eta_{\alpha \beta \gamma \delta}
$$

où l'on a posé:

$$
2 L_{\alpha \beta, \gamma \delta}=K_{\alpha \beta, \gamma \delta}^{*}+K_{\gamma \delta, \alpha \beta}^{*} .
$$

Par échange de $\alpha$ et $\gamma, \beta$ et $\delta$ dans (26.5) on voit que (26-3) est satisfaite.

Considérons maintenant le tenseur:

$$
K_{\alpha \beta, \gamma \delta}^{* *}=\frac{1}{4} \eta_{\alpha \beta, \mu \mu}^{z} \eta_{\gamma \delta \rho \sigma} K^{\lambda \mu, \rho \sigma}=\frac{1}{2} \eta_{\alpha \beta\rangle, \mu} K_{\gamma \delta,}^{*}{ }^{\lambda \mu} .
$$

De (26-3) il résulte:

$$
K_{\alpha \beta, \gamma \delta}^{* *}=\frac{1}{4} \eta_{\alpha \beta \lambda \mu} \eta^{\lambda \mu \rho \sigma} K_{\gamma \delta, \rho \sigma}=-\frac{1}{2} \varepsilon_{\alpha \beta}^{\rho \sigma} K_{\rho \sigma, \gamma \delta} .
$$

Soit :

$$
\boldsymbol{K}_{\alpha \beta, \gamma^{\delta}}^{* *}=-K_{\alpha \beta, \gamma \delta} \cdot
$$

De la relation (26-6) on peut tirer une formule importante pour la suite. A cet effet formons la quantité :

$$
K^{* * \alpha \beta, \gamma \lambda} K_{\alpha \beta, \gamma \mu}^{* *}=\frac{1}{16} \eta^{\alpha \beta \rho \sigma} \eta^{\gamma \lambda \tau \tau \eta_{\alpha \beta \beta \psi} \eta_{\gamma \mu \chi \pi} \pi} K_{\rho \sigma, \tau u} K^{\varphi \psi}, \chi^{\pi} .
$$

En introduisant les tenseur indicateurs de KRONECKER on a:

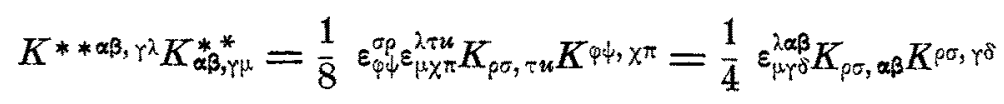

soit

$$
K^{* * \alpha \beta, \gamma \lambda} K_{\alpha \beta, \gamma \mu}^{* *}=\frac{1}{2} \delta_{\mu}^{\lambda} K^{\rho \sigma, \alpha \beta} K_{\rho \sigma, \alpha \beta}-K^{\rho \sigma, \lambda \delta} K_{\rho \sigma, \mu \delta} .
$$

On obtient ainsi :

$$
K^{* * \alpha \beta, \gamma^{\lambda}} K_{\alpha \beta, \gamma \mu}^{* *}+K^{\alpha \beta, \gamma \lambda} K_{\alpha \beta, \gamma \mu}=\frac{1}{2} \delta_{\mu}^{\lambda} K^{\alpha \beta, \gamma \delta} K_{\alpha \beta, \gamma \delta}
$$


De (26-6) il résulte alors:

$$
K^{\alpha \beta, \gamma^{\lambda}} K_{\alpha \beta, \gamma^{\mu}}=\frac{1}{4} \delta_{\mu}^{\lambda} K^{\alpha \beta, \gamma^{\delta}} K_{\alpha \beta, \gamma^{\delta}} .
$$

$2^{\circ}$ ) Etant donné un vecteur unitaire $\vec{u}$, associons au tenseur $K_{\alpha \beta, \gamma \delta}$ les deux tenseurs symétriques

$$
E_{\alpha \beta \beta}(\vec{u})=K_{\alpha, \beta \sigma \sigma} u \rho u^{\sigma} \quad H_{\alpha \beta}(\vec{u})=-K_{\alpha \rho \beta \sigma \sigma}^{*} u^{\rho} u^{\sigma} .
$$

qui satisfont manifestement à :

$$
E_{\alpha \beta} u^{\beta}=0 \quad H_{\alpha \beta} u^{\beta}=0 .
$$

La donnée de ces deux tenseurs détermine complètement le tenseur $K_{\alpha \beta, \gamma \delta}$ supposé satisfaire à (26-1). En effet adoptons un repère orthonormé tel que $\overrightarrow{e_{0}}=\vec{u} ; F$ et $H$ sont les tenseurs d'espace de composantes:

$$
E_{r s}=K_{r 0, s 0} \quad H_{r s}=-K_{r 0, s 0}^{*} \quad(r, s, \ldots,=1,2,3)
$$

et l'on voit que les composantes $K_{r s, t u}$ sont fournies par le tenseur $E$ ', les composantes $K_{r s, t_{0}}$ par le tenseur $H$. Evaluons en particulier

$$
A=\frac{1}{8} K^{\alpha \beta, \Upsilon^{\delta}} K_{\alpha \beta, \gamma^{\delta}} \text {. }
$$

Il vient:

$$
A=\frac{1}{2} K^{r 0, s 0} K_{r 0, s 0}+\frac{1}{2} K^{r s, t 0} K_{r 8, t 0}+\frac{1}{8} K^{r s, t u} K_{r s, t w}
$$

Or ;

D'autre part:

$$
K^{r 0,80} K_{r 0,80}=E^{r s} E_{r s} .
$$

$$
K^{r s t 0} K_{r s, t_{0}}=\eta^{r s w 0} \eta_{i r s t 0} K_{w 0,}^{* t_{0}} K^{* v 0}{ }_{, t_{0}}=-2 \delta_{v}^{u} K_{w 0,}^{*{ }^{t 0}} K^{*{ }^{* 0}, t_{0}}=-2 H^{r 8} H_{r s},
$$

Enfin

$$
K^{r s, t u} K_{r s, t u}=K^{* * r s, t u} K_{r s, t u}^{* *}=\eta^{r s v 0} \eta^{t u r v 0} \eta_{r s p o} \eta_{t u q 0} K_{v 0, w 0} K^{p 0, q 0}
$$

soit:

$$
K^{r s, t u} K_{r s, t u}=4 K^{v 0, w^{v 0}} K_{v 0, w_{0}}=4 E^{r s} E_{r s} .
$$

Il en résulte:

$$
A=E^{r s} E_{r s}-H^{r s} H_{r s} .
$$

$3^{\circ}$ ) Le tenseur d'ordre 4 qui apparait au $\$ 23$ et le tenseur analogue pour une radiation gravitationnelle pure ont conduit M. BEL [2] à associer à toute métrique satisfaisant:

$$
R_{\alpha \beta}-\lambda g_{\alpha \beta}=0
$$


le tenseur d'ordre 4:

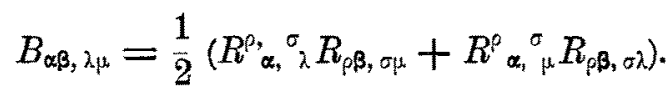

Ce tensenr est symétrique en $\alpha, \beta$, en $\lambda$, $\mu$ et symétrique par rapport anx couples $\alpha, \beta$ et $\lambda, \mu$. Evaluons sa dérivée covariante contractée. De:

$$
\nabla_{\alpha} R_{\beta, \gamma_{\delta}}^{\alpha}=0
$$

conséquence de (26-12), il résulte:

$$
2 \nabla_{\alpha} B_{\beta, \lambda \mu}^{\alpha}=R_{\lambda}^{\rho \alpha, \sigma} \nabla_{\alpha} R_{\rho \beta, \sigma \mu}+R^{\rho \alpha, \sigma} \nabla_{\alpha} R_{\rho \beta, \sigma \lambda} .
$$

Soit :

$$
4 \nabla_{\alpha} B_{\beta, \lambda \mu}^{\alpha}=R^{\rho \alpha, \sigma}{ }_{\lambda}\left(\nabla_{\alpha} R_{\beta \beta, \sigma \mu}-\nabla_{\rho} R_{\alpha \beta, \sigma \mu}\right)+R^{[\alpha, \sigma}{ }_{\mu}\left(\nabla_{\alpha} R_{\beta \beta, \sigma \lambda}-\nabla_{\rho} R_{\alpha \beta, \sigma \lambda}\right) .
$$

Ainsi, en vertu de l'identité de Brañohr :

Soit

$$
4 \nabla_{\alpha} B_{\beta, \lambda \mu}^{\alpha}=R^{c \alpha, \sigma} \nabla_{\beta} R_{\beta \alpha, \sigma \mu}+R_{\mu}^{r \alpha, \sigma} \nabla_{\beta} R_{\rho \alpha, \sigma \lambda} .
$$

$$
4 \nabla_{\alpha} B_{\beta, \lambda \mu}^{\alpha}=\nabla_{\beta}\left(R^{p \alpha, \sigma}{ }_{\lambda} R_{\rho \alpha, \sigma \mu}\right)
$$

De (26-8) il résulte ainsi :

$$
\nabla_{\alpha} B_{\beta, \lambda \mu}^{\alpha}=\frac{1}{2} \nabla_{\beta}\left(A g_{\lambda \mu}\right) \quad\left(\text { avec } A=\frac{1}{8} R^{\alpha \beta, \gamma \delta} R_{\alpha \beta, \gamma \delta}\right) .
$$

Ainsi le tenseur:

$$
T_{\alpha \beta, \lambda \mu}=B_{\alpha \beta, \lambda \mu}-\frac{1}{2} A g_{\alpha \beta} g_{\lambda \mu}
$$

qui admet les mêmes propriétés de symétrie que $B$, satisfait à l'identité de conservation :

$$
\nabla_{\alpha} T_{\beta, \lambda \mu}^{\alpha}=0 \text {. }
$$

Pour une radiation gravitationnelle pure $\left(R_{\alpha \rho}=0\right), T_{\alpha \beta . \lambda \mu}$ se réduit au tenseur $\tau_{\alpha \beta \lambda \mu} \mathrm{du} \& 25$. Si $\vec{u}$ est un vecteur unitaire, étudions la quantité :

$$
T \overrightarrow{(u)}=T_{\alpha \beta, \lambda, \beta} u^{\alpha} u^{\beta} u^{\lambda} u^{\mu}
$$

Dans un repere orthonormé tel que $\overrightarrow{e_{0}}=\vec{u}$

$$
T \overrightarrow{(u)}=T_{00,00}=B_{00,00}-\frac{1}{2} A=R_{0,0}^{\rho_{0}{ }_{0} R_{\rho 0, \sigma 0}}-\frac{1}{2} A=E^{r s}(\vec{u}) E_{r s}(\vec{u})-\frac{1}{2} A
$$

soit :

$$
T \overrightarrow{(u)}=\frac{1}{2}\left|E^{r s}(\vec{u}) E_{r s}(\vec{u})+H^{r s}(\vec{u}) H_{r s}(\vec{u})\right|>0
$$


A. Lichnerowicz: Ondes et radiations élcctromagnétiques, etc.

Ainsi $T(u)$ est strictement positive et n'est nulle que si le tenseur de courbure est nul. Le tenseur $T_{\alpha \beta, \lambda_{\mu}}$ semble ainsi généraliser dans ce cas le ten. senr de Maxwell.

\section{Construction d'un exemple de radiation.}

a) Supposons un voisinage $U$ de $V_{m+1}$ muni de la métrique:

$$
d s^{2}=g_{00}\left(x^{i}\right)\left(d x^{0}\right)^{2}+d s^{* 2} \quad\left(g_{00}=-\xi^{2}<0 ; i=1, \ldots, m\right)
$$

où $d s^{* 2}$ est une forme quadratique en les variables $\left(x^{i}\right)$. La métrique $d s^{2}$ admet un groupe à 1 paramètre d' isométries $x^{0} \rightarrow x^{0}+$ const. dont le générateur $\vec{\xi}$ a pour composantes $\left(\xi^{0}=1, \xi^{i}=0\right)$. La métrique quotient est $d \varepsilon^{* 2}$ et le tenseur antisymétrique $F_{i j}$ du $\S 19$ est nul. Si $\Gamma_{\beta \gamma}^{\alpha}(\alpha, \ldots,=0,1, \ldots, m)$ et $\Gamma_{j k}^{* i}$ sont les coefficients des connexions riemanniennes de $V_{n+1}$ et $V_{m}$ relativement aux coordonnées envisagées:

$$
\Gamma_{j k}^{* i}=\Gamma_{j k}^{i}
$$

Avec les notations du $\S 19$, le tenseur antisymétrique $F_{i j}$ est nul et si l'on rapporte $U$ à des repères orthonormés adaptés, les formules (19-14), (19-15) et (19.16) deviennent :

$$
\begin{aligned}
& R_{i j, k l}=R_{i j, k l}^{*} \\
& R_{i j, k_{0}}=0 \\
& R_{i 0, k_{0}}=\bar{\xi}^{\prime} \nabla_{k}^{*}\left(\partial_{i} \xi\right) .
\end{aligned}
$$

Nous allons utiliser les formules précédentes pour la construction d'un exemple de radiation.

b) L'espace numérique $R^{4}$ etant rapporté aux coordonnées:

$$
t=x^{0} \quad x=x^{1} \quad y=x^{2} \quad z=x^{3} \quad(\alpha \ldots=0,1,2,3)
$$

posons

$$
u=t-x=x^{0}-x^{1}
$$

et munissons un domaine $D$ de la métrique définie par:

$$
d s^{2}=e^{2}\left(d t^{2}-d x^{2}\right)-\left(\xi^{2} d y^{2}+\eta^{2} d z^{2}\right)=g_{\alpha \beta} d x^{\alpha} d x^{\beta}
$$

où $\varphi, \xi>0, \eta>0$ sont trois fonctions de la seule variable $u$. Etant donnée une fonction $f(u)$, nous désignerons par $f^{\prime}$ sa dérivée en $u$; on a alors:

et

$$
\partial_{0} f=f^{\prime} \quad \partial_{1} f=-f^{\prime} \quad \partial_{A} f=0 \quad(A, \ldots,=2,3)
$$

$$
\partial_{00} f=f^{\prime \prime} \quad \partial_{01} f=-f^{\prime \prime} \quad \partial_{11} f=f^{\prime \prime} \quad \partial_{A \alpha} f=0 .
$$


A. Lichnerowıcz: Ondes et radiations électromagnétiques, etc.

La métrique (27.2) admet deux groupes à 1 paramètre d'isométries définis par les deux générateurs

$$
\xi^{0}=\xi^{1}=\xi^{3}=0 \quad \xi^{2}=1
$$

et

$$
\eta^{0}=\eta^{1}=\eta^{2}=0 \quad \eta^{3}=1
$$

et satisfait de deux façons aux hypothèses du paragraphe précédent. Les nombres $\xi$ et $\eta$ sont les nombres scalaires associés aux denx générateurs.

Considérons la décomposition en carrés $d u d s^{2}$

$$
d s^{2}=\left(\theta_{-}^{9}\right)^{2}-\left(\theta_{-}^{1}\right)^{2}-\left(\theta_{-}^{2}\right)^{2}-\left(\theta_{-}^{3}\right)^{2}
$$

avec

$$
\theta_{-}^{0}=e^{\varphi} d x^{0} \quad \theta_{-}^{q}=e^{\varphi} d x^{1} \quad \theta_{-}^{2}=\xi d x^{2} \quad \theta_{-}^{3}=\eta d^{3} .
$$

Nous definissons ainsi des repères orthonormés adaptés à la fois aux deux groupes d'isométries. La matrice $\left(A_{\beta}^{\alpha}\right)$ de passage des coordonnées $\left(x^{\beta}\right)$ anx repères orthonormés est diagonale et a pour éléments :

$$
A_{\frac{0}{0}}^{\circ}=e^{\varphi} \quad A_{\frac{1}{4}}=e^{\varphi} \quad A_{\frac{2}{2}}=\xi \quad A_{\frac{3}{3}}^{\frac{3}{3}}=\eta
$$

la matrice inverse admettant comme seuls élëments nonnuls

$$
A_{\underline{0}}^{0}=e^{-\varphi} \quad A_{\underline{1}}^{l}=e^{-\varphi} \quad A_{\underline{2}}^{2}=\bar{\xi}_{\underline{\xi}}^{1} \quad A_{\underline{z}}^{3}=\bar{\eta}^{1} .
$$

c) Nous nous proposons de ealouler les composantes du tenseur de conrbure de (27.2) par rapport aux repères orthonormés envisagés.

Des formules préliminaires il résulte que si l' an exactement des indices $\alpha, \beta, \lambda, \mu$ a la valeur 2 ou la valeur $3, R_{\alpha \beta, \lambda \mu}=0$.

On obtient ainsi:

$$
\begin{aligned}
& R_{23,31}=0 \quad R_{23,12}=0 \quad R_{23,10}=0 \quad R_{23,20}=0 \quad R_{23,30}=0 \\
& R_{31,12}=0 \quad R_{31,10}=0 \quad R_{31,20}=0 \\
& R_{12,10}=0 \quad R_{12,80}=0 \\
& R_{10,20}=0 \quad R_{10,30}=0 \\
& R_{20, s 0}=0 \text {. }
\end{aligned}
$$


Avec les notations du 22, les composantes du tenseur de courbure peuvent etre rangées en la matrice:

$$
\left(R_{\Gamma J}\right)=\left(\begin{array}{cccccc}
R_{11} & 0 & 0 & 0 & 0 & 0 \\
0 & R_{22} & 0 & 0 & 0 & R_{26} \\
0 & 0 & R_{33} & 0 & R_{35} & 0 \\
0 & 0 & 0 & R_{44} & 0 & 0 \\
0 & 0 & R_{35} & 0 & R_{55} & 0 \\
0 & R_{28} & 0 & 0 & 0 & R_{68}
\end{array}\right)
$$

Il nons faut évaluer maintenant les différents éléments figurant encore dans $(27 \cdot 6)$.

Tout d'abord l'isométrie $x^{2} \rightarrow x^{2}+$ const. de $d s^{2}$ nous donne par passage au quotient la métrique:

$$
d s^{* 2}=e^{2 \varphi}\left(d t^{2}-d x^{2}\right)-\eta^{2} d z^{2}
$$

qui admet encore l'isométrie $x^{3}=x^{3}+$ const., ce qui nous conduit à la nouvelle métrique:

$$
d s^{* * 2}=e^{2 \varphi(\omega)}\left(d t^{2}-d x^{2}\right)=e^{2 \varphi(x)} d u d(t+x)
$$

laquelle est manifestement euclidienne. Des formules préliminaires, il résulte arec des notations évidentes:

$$
R_{10,10}=R_{10,10}^{*}=R_{10,10}^{* *} .
$$

On a ainsi:

$$
R_{10,10}=0
$$

Evaluons maintenant $R_{28,28}$. De (26.5), il résulte:

$$
R_{23,23}=\bar{\xi}^{1} \stackrel{\nabla}{\underline{B}}^{*}\left(\partial_{3} \xi\right)
$$

Il vient d'après (27-4)

$$
R_{23,23}=\xi \stackrel{*}{\nabla}_{3}\left(\partial_{3} \xi\right)
$$

$\xi$ ne dépendant que de $u$ et compte-tenu des formules préliminaires, on $a$ :

$$
\vec{\nabla}_{\mathrm{s}}^{*}\left(\partial_{3} \xi\right)=-\left(\Gamma_{33}^{0}-\Gamma_{33}^{1}\right) \xi^{\prime}
$$

Or:

$$
\Gamma_{33}^{0}=g^{00}[33,0]=-\frac{1}{2} g^{00} g_{33}^{\prime} \quad \Gamma_{33}^{1}=g^{11}[33,1]=-\frac{1}{2} g^{00} g_{33}^{\prime} .
$$


Il en résulte $R_{23,23}=0$ et par suite

$$
R_{23,23}=0
$$

d) On a de même:

$$
R_{12,12}=\bar{\xi}^{1} \nabla_{1}^{*}\left(\partial_{1} \xi\right)
$$

soit d'après (27-4)

$$
R_{12,12}=\xi \stackrel{*}{\nabla}{ }_{1}(\partial, \xi)
$$

En explicitant la dérivation covariante, il vient:

$$
R_{12,12}=\xi\left[\xi^{\prime \prime}-\left(\Gamma_{11}^{0}-\Gamma_{11}^{\prime}\right) \xi^{\prime}\right]
$$

Or

$$
\Gamma_{11}^{0}=g^{00}[11,0]=\frac{1}{2} g^{00} g_{00}^{\prime}=\varphi^{\prime} \quad \Gamma_{11}^{4}=g^{11}[11,1]=-\frac{1}{2} g^{00} g_{00}^{\prime}=-\varphi^{\prime}
$$

On en déduit:

$$
R_{12,12}=\xi\left(\xi^{\prime \prime}-2 \varphi^{\prime} \xi^{\prime}\right)
$$

et d'après (27.5)

$$
R_{12,12}=e^{-2} \bar{\varphi} \xi^{-1}\left(\xi^{\prime \prime}-2 \varphi^{\prime} \xi^{\prime}\right)
$$

D'autre part:

$$
R_{12,20}=-\underline{\xi \nabla} \nabla_{1}\left(\partial_{0} \xi\right)
$$

C'est-à-dire :

$$
R_{2,20}=-\xi \nabla_{1}\left(\partial_{0} \xi\right)=\xi\left[\xi^{\prime \prime}+\left(\Gamma_{10}^{0}-\Gamma_{10}^{4}\right) \xi^{\prime}\right] .
$$

Or:

$$
\Gamma_{10}^{0}=g^{00}[10,0]=-\frac{1}{2} g^{00} g_{00}^{\prime}=-\varphi^{\prime} \quad \Gamma_{10}^{t}=g^{11}[10,1]=\frac{1}{2} g^{00} g_{00}^{\prime}=\varphi^{\prime} .
$$

On obtient:

$$
\left.R_{12,20}=R_{20,20}=e^{-2 \varphi \xi^{-1}\left(\xi^{\prime \prime}\right.}-2 \varphi^{\prime} \xi^{\prime}\right) .
$$

En ce qui concerne la matrice (27.6), nous arons dono ètabli:

$$
R_{11}=R_{44}=0
$$

et

$$
R_{35}=R_{35}=R_{55}=e^{-2 \varphi \xi^{-1}}\left(\xi^{\prime \prime}-2 \varphi^{\prime} \xi^{\prime}\right)
$$


A. Lichnerowicz: Ondes et radiations électromagnétiques, etc.

En jouant sur l'autre groupe d'isométries, on établirait de même:

$$
\left.R_{22}=-R_{26}=R_{66}=e^{-z_{\varphi}^{-1}} \eta^{\prime \prime} \eta^{\prime \prime}-2 \varphi^{\prime} \eta^{\prime}\right)
$$

Si nous posons:

$$
\left.\left.a=e^{-2 \varphi \xi\left(\xi^{\prime \prime}\right.}-2 \varphi^{\prime} \xi^{\prime}\right) \quad b=e^{-2 \varphi \eta\left(\eta^{\prime \prime}\right.}-2 \varphi^{\prime} \eta^{\prime}\right)
$$

la matrice $\left(R_{I J}\right)$ prend la forme (21-27). Il en résulte que le tenseur de courbure $R_{\alpha \beta, \gamma \delta}$ de $(27.2)$ et le vectenr $l_{\alpha}$ de composantes

$$
l_{0}=1 \quad l_{1}=-1 \quad l_{A}=0
$$

qui est le gradient de $u$ satisfont aux relations (24-1), (24.2). Il existe par suite un scalaire $\tau$ tel que:

$$
R_{\alpha \beta}=\tau l_{\alpha} l_{\beta}
$$

Ainsi la métrique (27-2) représente aux différents points du domaine $D$ où elle est régulière, un état de radiation totale pure de type intégrable. Pour qu'on ait affaire à une radiation gravitationnelle pure, il faut et il suffit que $a+b=0$, c'est-à-dire que $\xi$ et $\eta$ satisfassent à la relation:

$$
\bar{\eta}\left(\eta^{\prime \prime}-2 \varphi^{\prime} \eta^{\prime}\right)+\bar{\xi}^{-1}\left(\xi^{\prime \prime}-2 \varphi^{\prime} \xi^{\prime}\right)=0 \text {. }
$$

Cet état ne sera pas trivial si le tenseur de courbure envisagé n'est pas nul, e'est-à-dire si la relation:

$$
\bar{\eta}^{-1}\left(\eta^{\prime \prime} 2 \varphi^{\prime} \eta^{\prime}\right)-\bar{\xi}^{-1}\left(\xi^{\prime \prime}-2 \varphi^{\prime} \xi^{\prime}\right)=0
$$

n'est pas en outre satisfaite. Posons:

$$
\xi \eta=e^{\alpha} \quad \eta / \xi=e^{2 \beta} .
$$

La relation (27.16) prend la forme:

$$
\alpha^{\prime \prime}+\alpha^{\prime 2}+\beta^{\prime 2}-2 \alpha^{\prime} \varphi^{\prime}-0
$$

et la relation $(27-17)$ la forme:

$$
\beta^{\prime \prime}+2 \alpha^{\prime} \beta^{\prime}-2 \beta^{\prime} \varphi^{\prime}=0 .
$$

e) Remarquons que, dans le cas où la métrique (27-2) est euclidienne, on a:

$$
2 \varphi^{\prime}=\frac{\xi^{\prime \prime}}{\xi^{\prime}}=\frac{\eta^{\prime \prime}}{\eta^{\prime}} .
$$


Par intégration, on en déduit $q u^{\prime}$ il existe deux constantes $C_{1}$ et $C_{2}$ telle que:

$$
C_{1} \xi+C_{2} \eta=\text { const. }
$$

Si $\xi$ et $\eta$ sont elles-mêmes des constantes, $\varphi$ peut ètre choisie arbitrairement. S'il nen est pas ainsi:

$$
e^{2 \varphi}=C_{8} \xi
$$

\section{8. - Forme de Rosen pour la métrique.}

Un $d s^{2}$ du type (27.2) a eté introduit en 1937 par Roskn $\left({ }^{9}\right)$ dans une optique différente. Mais il est possible de ramener (27.2) à la forme indiquée par Rosen. L'expression (27-2) de notre $d s^{2}$ admettant en effet un changement arbitraire $u \rightarrow f(u)$, il est possible d'utiliser ce fait pour simplifier la forme de notre métrique.

a) Si $\xi_{\eta}=$ const., soit $\alpha=$ const, il résulte de (27-18) que dans le cas gravitationnel $\left(R_{\alpha \beta}=0\right.$ ), on a alors $\eta / \xi=$ const. soit $\xi=$ const., $\eta=$ const. et et le $d s^{2}$ envisagé est nécessairement euclidien. Nous choisirons comme variable $u$ celle définie par:

$$
\xi \eta=u^{2}
$$

et poserons:

$$
v=t+x
$$

Considérons la partie $\left(R^{4}\right)^{+}$de $R^{ \pm}$définie par:

$$
u=t-x>0 .
$$

Sur cette partie la métrique définie par:

$$
d s^{2}=e^{2 \varphi}\left(d t^{2}-d x^{2}\right)-u^{2}\left(e^{-2 \beta} d y^{2}+e^{2 \beta} d z^{2}\right)
$$

est régulière si les fonctions $\beta(u)$ et $\varphi(u)$ sont pour $u>0$, de classe $\left(C^{1}, C^{s}\right.$ par morceaux). Nous avons alors:

$$
\alpha=\log u \quad \alpha^{\prime}=\frac{1}{u} \quad \alpha^{\prime \prime}=-\frac{1}{u^{2}} .
$$

Pour que (28.1) satisfasse aux équations $R_{\alpha \beta}=0$, il faut et il suffit que:

$$
2 \varphi^{\prime}=u \beta^{\prime 2} \text {. }
$$

(9) Rosen, "Phys. Z. Sotjer Union ", 12, p. 366 (1937); Eixstein et Rosen J. «Franklin Inst. ", 223, $43,(1937)$. 
Nous ponvons alors choisir arbitrairement pour $\beta(u)$ une fonction de classe $\left(C^{1}, C^{3}\right.$ par morcaux) pour $u>0$ et $\varphi$ se trouvera déterminé par quadrature ì partir de la relation (28.2). La métrique obtenue sur $\left(R^{4}\right)^{+}$est à tenseur de courbure non nulle si la relation:

$$
u \beta^{\prime \prime}+2 \beta^{\prime}-u^{2} \beta^{\prime 3}=0
$$

n'est pas satisfaite par $\beta$. En particulier en un point où $\beta^{\prime}=\beta^{\prime \prime}=0$ le tenseur de courbure est nul.

Nous arons ainsi obtenu la métrique indiquée par Rosen, mais qui n'est valable que pour $\left(R^{4}\right)^{+}$, ce qui semblait en limiter l'interêt.

b) En adoptant d'autres coordonnées, il est aisé de munir $R^{t}$ d'une métrique partont régulière, euclidienne dans certains domaines et coincidant dans d'autres domaines avec la métrique de ROSEN.

En un point où $\beta^{\prime}(u)=0$, on peut ramener (28-1), soit:

$$
d s^{2}=e^{2 \varphi} d u d v-u^{2}\left(e^{-i \beta} d y^{2}+e^{i \beta} d z^{2}\right)
$$

à la forme galiléenne. Adoptons à cet effet les coordonnées $u$, $v$ et

$$
\bar{y}=u e^{-\beta} y \quad \bar{z}=u e^{\beta} z .
$$

Par différentiation, il vient:

$$
u e^{-\beta} d y=d \bar{y}-\frac{\bar{y}}{u} d u \quad \cdot u e^{\beta} d z=d \bar{z}-\frac{\bar{z}}{u} d u .
$$

On en déduit:

$$
u^{\prime}\left(e^{-: \beta} d y^{2}+e^{2 \beta} d z^{2}\right)=d \bar{y}^{2}+d \bar{z}^{2}-2 \frac{\bar{y} d \bar{y}+\bar{z} d \bar{z}}{u} d u+\frac{\bar{y}^{2}+\bar{z}^{2}}{u^{2}} d u^{2}
$$

et

$$
d s^{2}=d u\left(e^{2} \Phi d v+2 \frac{\bar{y} d \bar{y}+\bar{z} d \bar{z}}{u}-\frac{\bar{y}^{2}+\bar{z}^{2}}{u^{2}} d u\right)-\left(d \bar{y}^{2}+\overline{d z}^{2}\right)
$$

En effectuant le nouveu changement de variables

$$
\bar{u}=u \quad \bar{v}=e^{2 \varphi} \cdot v+\frac{\bar{y}^{2}+\bar{z}^{2}}{u}
$$

il vient an pointe considéré, comple tena de (28-2):

$$
d s^{2}=d \bar{u} d \bar{v}-\left(\overline{d y^{2}}+d \bar{z}^{2}\right)
$$

et il suffit de poser $\bar{u}=\bar{t}-\bar{x}, \bar{v}=\bar{t}+\bar{x}$, pour se trouver ramené à la forme galiléenne. 
Cela posé, supposons que $\beta$ soit une fonction quelconque de classe $\left(C^{1}\right.$, $C^{3}$ par morceaux) et effectuons sur (28-4) la transformation $\left({ }^{10}\right)$ definie par:

$$
\bar{u}=u, \quad \bar{v}=e^{2 \varphi} v+\frac{\bar{y}^{2}+\bar{z}^{2}}{u} \quad \bar{y}=u e^{-\beta} y \quad \bar{z}=u e^{\beta} z .
$$

Cette transformation définit une application biunivoque, à jacobien non nul, de $\left(R^{4}\right)^{+}$sur lui-même. il vient:

$$
u e^{-\beta} d y=d \bar{y}-\frac{\bar{y}}{u} d u+\beta^{\prime} \bar{y} d u \quad u e^{\beta} d z=d \bar{z}-\frac{\bar{z}}{u} d u-\beta^{\prime} \bar{z} d u .
$$

Par suite:

$$
\begin{gathered}
u^{2}\left(e^{-2 \beta} d y^{2}+e^{2 \beta} d z^{2}\right)=d \bar{y}^{2}+d \bar{z}^{2}-2 \frac{\bar{y} d \bar{y}+\bar{z} d \bar{z}}{u} d u+\frac{\bar{y}^{2}+\bar{z}^{2}}{u^{2}} d u^{2} \\
+2 \beta^{\prime}\left(\bar{y} d \bar{y}-\bar{z} d \bar{z}-\frac{\bar{y}^{2}-\bar{z}^{2}}{u} d u\right) d u+\beta^{\prime 2}\left(\bar{y}^{2}+\bar{z}^{2}\right) d u^{2}
\end{gathered}
$$

D'autre part:

$$
d \bar{v}=e^{2} \varphi d v+2 \frac{\bar{y} d \bar{y}+\bar{z} d \bar{z}}{u}-\frac{\bar{y}^{2}+\bar{z}^{2}}{u^{2}} d u+\beta^{\prime 2}\left[u \bar{v}-\left(\bar{y}^{2}+\bar{z}^{2}\right)\right] d u
$$

On en déduit:

$$
d s^{2}=d u d \bar{v}-\left(d \bar{y}^{2}+d \overline{z^{2}}\right)-2 \beta^{\prime}\left(\bar{y} d \bar{y}-\bar{z} d \bar{z}-\frac{\bar{y}^{2}-\bar{z}^{2}}{u} d u\right) d u-\beta^{\prime 2} u \bar{v} d u^{2}
$$

$u_{0}$ et $u_{1}$ étant deux nombres positif, choisissons pour $\beta^{\prime}$ une fonction définie et de classe $\left(C^{1}, C^{2}\right.$ par morceaux $)$ pour $u_{0} \leq u \leq u_{1}$ et telle que:

$$
\beta^{\prime}\left(u_{0}\right)=\beta^{\prime}\left(u_{1}\right)=0 \quad \beta^{\prime \prime}\left(u_{0}\right)=\beta^{\prime \prime}\left(u_{1}\right)=0 .
$$

Considérons l'espace numerique $R^{\sharp}$ des ensemble $(u, \bar{v}, \bar{y}, \bar{z})$ et munissons-le de la métrique définie de la manière suivante:

$$
\begin{array}{lr}
\text { pour } \quad u \leq u_{0} & d s^{2}=d u d \bar{v}-\left(d \bar{y}^{2}+d \bar{z}^{2}\right) \\
\text { pour } u_{0} \leq u \leq u_{1} & \text { le } d s^{2} \text { est donné par }(28 \cdot 8) \\
\text { pour } \quad u \geq u_{1} & d s^{2}=d u d \bar{v}-\left(d \bar{y} \bar{y}^{2}+d \bar{z}^{2}\right) .
\end{array}
$$

La métrique ainsi définie sur $R^{4}$ satisfait aux équations $R_{\alpha \beta}=0$ et est même partout $d \theta$ classe $C^{2}$. Elle n'est pas euclidienne pour $u_{0}<u<u_{1}$ si $\beta^{\prime}$ ne satisfait pas (28-3).

Dans cette région, elle représente un état de radiation gravitationnelle pure

(10) Cette transformation et le raisonnement qui suit sont dûs à BoNDI, « Nature», t. 179, 1072, (1957). 
et est de type intégrable. Bien entendu, elle n'est pas euclidienne dans le domaine à l'infini de l'espace.

\section{Formules de déviation}

\section{9. - Formule de la déviation geodesique.}

Pour étndier les effets physique soit de l'existence d' un front d'onde gravitationnelle avec discontinuités du tensenr de courbure, soit d'une état de radiation, nous utiliserons les formules dites de la deviation géodesique. Dans un donaine $\Delta$ de $V$, considérons les trajectoires spatio-temporelles d'une famille de particnles d'essai. Chacune de ces trajectoires est une géodesique de $V_{4}$ orientée dans le temps. Nous nous proposons d'étudier «l'accélération relative» (par rapport à $s$ ) de deux particnles infiniment voisines pour des points de $V_{4}$ définissant un vecteur orthogonal aux trajectoires.

a) Les considérations qui suivent sont natureilement locales. Donnons nous, dans un voisinage de $V_{4}$ une congruence de courbrs orientées dans le temps et désignons par $\vec{u}$ le vecteur unitaire tangent. De cette congruence, extrayons une famille a 1 parametre de courbes $\Gamma_{t} q$ ui engendre une surface $S$ de dimension 2. Sur chaque courbe $\Gamma_{t}$, nous utiliserons comme paramètre l'arc $s$ compté, à partir d'une origine convenable. La surface $S$ se trouve ainsi rapportée aux deux paramètres $s$ et $t$ et $x(s, t)$, où $x \in S$, sera supposé deux fois continuement differentiable par rapport à $(s, t)$. Le vecteur unitaire $\vec{u}$ tangent en $x$ à $\Gamma_{t}$ est le vecteur $\partial x / \partial s$ et nous désignons par $\vec{v}$ le vecteur $a x / \partial t$.

Le voisinage envisagé étant rapporté à des coordonnées locales $\left(x^{\alpha}\right)$, les vecteurs $u$ et $v$ ont respectivement pour composantes:

$$
u^{\alpha}=\frac{\partial x^{\alpha}}{\partial s} \quad v^{\alpha}=\frac{\partial x^{\alpha}}{\partial t}
$$

et

$$
\frac{\partial u^{\alpha}}{\partial t}=\frac{\partial v^{\alpha}}{\partial s}
$$

En terme de differentiation absolue, il vient:

$$
\frac{\bar{V} u^{\alpha}}{d t}=\frac{\partial u^{\alpha}}{\partial t}+\Gamma_{\beta \gamma}^{\alpha} u^{\beta} \frac{\partial x_{\gamma}^{\gamma}}{\partial t}=\frac{\partial v^{\alpha}}{\partial s}+\Gamma_{\beta \gamma}^{\alpha} u^{\beta} v_{\gamma}
$$

(I) Voir Prrani [2]. 
On a ainsi

$$
\frac{\bar{V} u^{\alpha}}{d t}=\frac{\bar{V} v^{\alpha}}{d s}
$$

Cela posé, appliquons à $\vec{u}$ l'identité de RIcor

$$
\bar{\nabla} \lambda \bar{V}_{\mu} u^{\alpha}-\bar{\nabla}_{\mu} \bar{V}_{\lambda} u^{\alpha}=R_{\rho, \lambda \mu}^{\alpha} u_{\rho} .
$$

Etudions le produit du premier membre par $v^{\lambda} u^{\mu}$. Il vient:

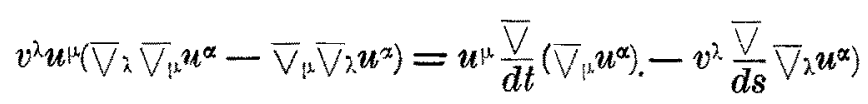

soit:

$$
\boldsymbol{v}^{\lambda} \boldsymbol{u}^{\mu}\left(\bar{\nabla}_{\lambda} \bar{\nabla}_{\mu} \boldsymbol{u}^{\alpha}-\bar{\nabla}_{\mu} \bar{\nabla}_{2} \boldsymbol{u}^{\alpha}\right)=\frac{\bar{\nabla}}{d t} \bar{\nabla}_{d s} \boldsymbol{u}^{\alpha}-\frac{\bar{V}}{d s} \frac{\bar{\nabla} \boldsymbol{u}^{\alpha}}{d t}-\frac{\bar{\nabla} \boldsymbol{u}^{\mu}}{d t} \bar{\nabla}_{\mu} \boldsymbol{u}^{\alpha}+\frac{\bar{\nabla} \boldsymbol{v}^{\lambda}}{d s} \overline{\mathrm{V}}_{\lambda} \boldsymbol{u}^{\alpha}
$$

De (29.2), il résulte:

$$
v^{2} u^{\mu}\left(\bar{\nabla}_{\lambda} \bar{\nabla}_{\mu} u^{\alpha}-\bar{\nabla}_{\mu} \bar{V}_{\lambda} u^{\alpha}\right)=\frac{\bar{V}}{d t} \frac{\bar{V} u^{\alpha}}{d s}-\frac{\bar{V}^{2} v^{\alpha}}{d s^{2}}
$$

De (29-3) on déduit ainsi la formule:

$$
\frac{\bar{V}^{2} v^{\alpha}}{d s^{2}}+R_{\rho, \lambda \mu}^{\alpha} u^{\rho} v^{\alpha} u^{\mu}=\frac{\bar{V}}{d t} \frac{\bar{V} u^{\alpha}}{d s}
$$

b) Supposons que les courbes $\Gamma_{t}$ envisagées soient des géodesiques de $V_{4}$. On a alors:

$$
\frac{\bar{\nabla} u^{\alpha}}{d s}=0
$$

Le vecteur $u^{\alpha}$ etant unitaire

$$
u_{\alpha} \frac{\bar{\nabla} u^{\alpha}}{d t}=u_{\alpha} \frac{\bar{\nabla} v^{\alpha}}{d s}=0
$$

et d'aprés (29.5)

$$
\frac{\bar{V}}{d s}\left(u_{\alpha} v^{\alpha}\right)=\frac{\bar{V} u_{\alpha}}{d s} v^{\alpha}+u^{\alpha} \frac{\bar{V} v^{\alpha}}{d s}=0
$$

Ainsi sur chaque courbe $\Gamma_{t}$ on a

$$
u_{\alpha} v^{\alpha}=\text { const. }
$$

Soit $C$ une trajectoire orthogonale sur $S$ des géodesiques $\Gamma_{t}$ et, pour origine de l'arc $s$ sur chaque $\Gamma$, adoptons un point de C. Aux points de $C(s=0) \vec{n}$ 
et $\vec{v}$ sont orthogonaux; par suite sur tout $S, u_{\alpha} v^{\alpha}=0$ et $\vec{v}$ est orthogonal aux géodesiques.

De (29.4) il résulte:

$$
\frac{\bar{V}^{2}}{d s^{2}} v^{\alpha}+R_{\rho}^{\alpha}, \lambda \mu \rho v^{\lambda} u^{\mu}=0
$$

C'est a cette formule qu'on donne le nom de fermule de l'écart géodesique. Les points correspondants sur deux géodesiques infiniment voisines étant cenx définis par la direction de $v^{\alpha}$ orthogonal aux géodesiques, elle nous fournit ce qu'on pent appeller l'accélération relative, par rapport à $s$, des deux particules d'essai qui décrivent ces géodesiques.

\section{0. - Applications.}

a) Au voisinage du point $\mathrm{x}$, considérons les trajectoires spatio-temporelles des particules d'essai envisagées, supposées soummis au seul champ de gravitation. Si $\Gamma$ est la trajectoire issue de $x$, introduisons en ce point un repère orthonormé $\left.\vec{e}_{\alpha}\right)$ tel que le premier vecteur $\overrightarrow{e_{0}}$ coincide arec le vecteur unitaire tangent en $x$ a $\Gamma$. Au point $x$ le vecteur $\vec{u}$ admet les composantes

$$
u^{0}=0 \quad u^{u}=0 \quad(u=1,2,3)
$$

et $\vec{v}$ etant orthogonal à $\vec{u}$ admet les composantes:

$$
v^{0}=0 \quad v^{\prime \prime} .
$$

Transportons par parallélisme le repère initial le long de $\Gamma$ et munissons le voisinage de $\Gamma$ d'une famille de repère prolongeant $C^{2}$ les repères ainsi attaohés anx different points de $\Gamma$. Si $\gamma^{\alpha} \beta_{\gamma}$ sont les coefficients de la connexion riemanienne de $V_{4}$ relativement à cette famille de repères, on a sur $\Gamma$ :

$$
\gamma^{\alpha} \beta_{\gamma} u r=0 \text {. }
$$

Evaluons aux points de $\Gamma$ les composantes de $\frac{\bar{V}^{2} v^{\alpha}}{d s^{2}}$. Il vient en ces points

$$
\frac{\bar{V} v^{\alpha}}{d s}=\frac{d v^{\alpha}}{d s}+\gamma_{\beta \gamma}^{\alpha} u v^{\beta}
$$

et par dérivation

$$
\frac{\bar{V}^{2} v^{\alpha}}{d s^{2}}=\frac{d^{2} v^{\alpha}}{d s^{2}}+\frac{d}{d s}\left(\gamma^{\alpha}{ }_{\beta \gamma} u \gamma v^{\beta}\right)+\gamma_{\beta \gamma}^{\alpha} u r \frac{\bar{V}^{2} v^{\beta}}{d s_{2}}
$$

Sur $\Gamma$ on a donc d'après (30-3)

$$
\frac{\bar{V}^{2} v^{\alpha}}{d s^{2}}=\frac{d^{2} v^{\alpha}}{d s^{2}}
$$


A. Lichnbrowicz: Ondes et radiations électromagnétiques, etc.

et l'équation (29.6) prend la forme

$$
\frac{d^{2} v^{\alpha}}{d s^{2}}+R_{\rho, \lambda \mu}^{\alpha} u \rho v^{\lambda} u^{\mu}=0
$$

b) Les trajectoires des particules d'essai soumises au seul champ de gravitation sont des géodesiques orientées dans le temps de $V_{4}$. Il en résulte qu'en tout point de $\Gamma, \overrightarrow{e_{0}}=\vec{u}$ et par suite $v^{0}=0$ sur $\Gamma$.

An point $x$ choisi, (30.5) s'écrit pour $\alpha=u$ :

$$
\frac{d v^{u}}{d s^{2}}+R_{0, r n}^{u} v^{v}=0
$$

et pour $\alpha=0$ nous n'obtenons, d'après la remarque précédente qu' une identité. Supposons $q$ u'il existe sur $V_{4}$ un front d'onde gravitationnelle passant par $x$, avec discontinuités du tenseur de courbure. De (30.6) il résulte:

$$
\left[\frac{d^{2} v^{u}}{d s^{2}}\right]=-\left[R^{u, v o}\right] v^{v}
$$

soit:

$$
\left[\frac{d^{2} v^{u}}{d s^{2}}\right]=K^{u} v^{v}
$$

en posant:

$$
K^{u}{ }_{v}=-\left[R^{u}{ }_{0, v 0}\right] .
$$

Le vecteur $\overrightarrow{e_{0}}$ etant fixé en $x$, nous avons du choisir le vecteur $\overrightarrow{e_{u}}$ du repère en ce point de façon que la matrice des discontinuités du tensenr de courbure prenne la forme $(22 \cdot 1)$. La matrice $\left(K^{u}{ }_{v}\right)$ peut alors s'écrire (avec $\rho=0$ ):

$$
\left(\boldsymbol{K}^{u_{v}}\right)=\left(\begin{array}{rrr}
0 & 0 & 0 \\
0 & \sigma & 0 \\
0 & 0 & -\sigma
\end{array}\right)
$$

et (30.10) peut être explicité par les relations:

$$
\left[\frac{d^{2} v^{1}}{d s^{2}}\right]=0 \quad\left[\frac{d^{\prime} v^{2}}{d s^{2}}\right]=\sigma v^{2} \quad\left[\frac{d^{2} v^{3}}{d s^{2}}\right]=-\sigma v^{3}
$$

qui nous donnent les composantes de la discontinuité de l'accélération rela. tive. La composante le long $\mathrm{de} \vec{e}_{1}$ c'est à dire dans la direction d'espace de la propagation de l'onde est toujours nulle. La discontinuité envisagèe dependant linéairement du vecteur $\vec{v}$ en $x$, nous pouvons étudier le cas général comme superposition des deux cas particuliers suivants:

Io, Si $\vec{v}$ est colineaire à $\overrightarrow{e_{1}}$, on a $v^{2}=v^{3}=0$ et la discontinuité de l'accélération relative est nulle. 
A. LrChNerowicz: Ondes et radiations électromagnétiques, etc.

$\left.2^{\circ}\right)$ Si $\vec{v}$ et dans le 2-plan $\left(\overrightarrow{e_{2}}, \overrightarrow{e_{3}}\right)$, e' est à dire orthogonale à la direction d'espace de la propagation de l'onde:

$$
v^{2}=v \cos \vartheta \quad v^{3}=v \sin \vartheta
$$

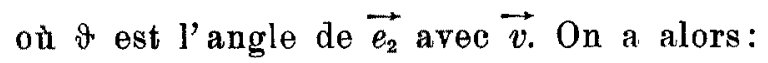

$$
\left[\frac{d^{2} v^{2}}{d s^{2}}\right]=\sigma v \cos \vartheta \quad\left[\frac{d^{2} v^{3}}{d s^{2}}\right]=-\sigma v \sin \vartheta
$$

et la discontinuité est portée par la direction du plan $\left(e_{2}, e_{3}\right)$ définie à partir de $\overrightarrow{e_{2}}$ par l'angle $-\vartheta$.

On a ainsi mis en évidence le caractère transversal de l'onde gravitationelle et mis en place la discontinuité de l'nocéleration relative produite par le front d'onde.

Dans un domaine de $V_{4}$ où la métrique représente un état de radiation gravitationelle pure, on voit sur (30-6) que les résultats sont identiques en ce qui concerne l'accélération relative elle-meme.

\section{Déviation des trayectoires de particules chargées en présence d'un champ électromagnétique.}

Supposons que dans un domaine $\triangle$ de $V_{4}$ existe un champ électroma. gnétique $F_{\alpha \beta}$ et proposons-nous d'étudier par analogie avec celle d'une géodesique, la déviation de la trajectoire d'une particule d'essai chargée qui se trouvera ainsi soumise à la fois au champ gravitationel et au champ électromagnétique. Les trajectoires spatio-temporelles des particules chargées satisfont au système differentiel qui s'exprime en cordonnées locales par:

$$
\frac{d^{2} x^{\alpha}}{d s^{2}}+\Gamma_{\beta \gamma}^{\alpha} \frac{d x^{\beta}}{d s} \frac{d x r}{d s}=k F_{\beta}^{\alpha} \frac{d x^{\beta}}{d s}
$$

où la constante $k$ caractérise le rapport de la charge à la masse de la particule envisagée.

a) Donnons nous sur $\triangle$ une poussière de particules chargées pour lesquelles le rapport $k$ est le mème et extrayons de la congruence de leurs trajectoires sur $\Delta$ une famille à 1 paramètre $t$ de trajectoires $\Gamma_{t}$. Les hypothéses, et les notations étant identiques à celles du $\S 29, a$, le système differentiel (31-1) pent s'écrive:

$$
\frac{\nabla u^{\alpha}}{d s}=k F_{\beta}^{\alpha} u^{\beta}
$$


On a d'autre part (29-4), soit:

$$
\frac{\nabla^{2} v^{\alpha}}{d s^{2}}+R_{\rho, \lambda \underline{\mu}}^{\alpha} u_{\rho}^{\rho} v^{\lambda} u^{\mu}=\frac{\nabla}{d t} \frac{\nabla u^{\alpha}}{d s}
$$

De (31-1) il résulte: $\frac{\nabla}{d t} \frac{\nabla u^{\alpha}}{d s}=k\left(\nabla_{\rho} F^{\alpha}{ }_{\beta} u^{\beta} v^{\rho}+F_{\beta}^{\alpha} \frac{\nabla v^{\beta}}{d s}\right)$.

Ainsi le vecteur $v^{\alpha}$ satisfait au système différentiel:

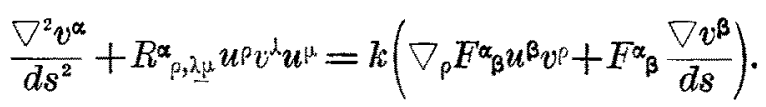

Soit $C$ une trajectoire orthogonale, sur la surface engendréc, des courbes $\Gamma_{t}$ envisagées. Adoptons pour origine de l'are, sur chaque $\Gamma_{t}$, un point de $C$. Aux points de $C(s=0) \vec{u}$ et $\vec{v}$ sont orthogonaux, mais il n'en est plus de même ici en dehors de $O$ puisque:

$$
\frac{d}{d t}\left(u_{\alpha} v^{\alpha}\right)=\frac{\nabla u^{\alpha}}{d s} v^{\alpha}=k F_{\alpha \beta} v^{\alpha} u^{\beta}
$$

n'est pas identiquement nul.

La formule (31.4) fournit l'accélération relative, par rapport à $s$, de deux particules d'essai infiniment voisines, les points correspondants des deux trajotoires spatio-temporelles étant ceux de même $s$, compté à partir de $C$.

b) $x$ etant un point determiné de l'une des trajectoires spatio-temporelles précédentes $I$, introduisons en ce point un repère orthonormé $\left(\overrightarrow{e_{\alpha}}\right)$ tel que le premier vecteur $\overrightarrow{e_{0}}$ coincide avec le vecteur unitaire $\vec{u}$ tangent en $x$ à $\Gamma$. D'apres une remarque précédente, nous pouvons supposer qu'en ce point $x, \vec{v}$ est orthogonal à $\vec{u}$. Ainsi en $x$, les vecteur $\vec{u}, \vec{v}$ admettent les composantes $(30-1)$ et $(30-2$.

En adoptant dans le voisinage de $\Gamma$ une famille de repères identiques à ceux definis au $\S 30, a,(31.4)$ prend la forme:

$$
\frac{d^{2} v^{\alpha}}{d s^{2}}+R_{\rho, \lambda \mu}^{\alpha} u^{\rho} v^{\lambda} u^{\mu}=k\left(\nabla_{\rho} F_{\beta}^{\alpha_{\beta}} u^{\beta} v^{\rho}+F^{\alpha} \frac{d v^{\beta}}{d s}\right)
$$

Supposons qu'il existe sur $V_{4}$ un front d'onde $S$ à la fois gravitationelle et électromagnétique passant par $x$; à la traversée de $S$ il $y$ a discontinuités du tenseur de courbure et $\mathrm{d} \mathbf{u}$ tenseur derivé du champ électromagnètique.

Nous supposons naturellement que la métrique satisfait à des équations d'Einstern à second membre contina et que $F_{\alpha \beta}$ satisfait aux équations de MAXwell avec vecteur-courant continu. De (31.5) il résulte $q u^{\prime}$ au point $x$ envisagé:

$$
\left[\frac{d^{2} v^{\alpha}}{d s^{2}}\right]+\left[R_{\rho, \lambda_{\mu}}^{\alpha}\right] u^{\rho} v^{\lambda} u^{\mu}=k\left[\nabla_{\rho} F_{\beta}^{\alpha_{\beta}}\right] u^{\beta} v_{\rho} \text {. }
$$


Pour $\alpha=0$ et compte-tenu du choix du repère en $x,(31-6)$ donne:

$$
\left[\frac{d^{2} v^{0}}{d s^{2}}\right]=0
$$

Pour $\alpha=u$ et compte-tenu des expressions (30.1) et (30-2) des composantes de $\vec{u}$ et de $\vec{v}$ :

$$
\left[\frac{d^{2} v^{u}}{d s^{2}}\right]=K^{w} v^{v}+k\left[\nabla_{v} F^{u}{ }_{0} v^{v}\right.
$$

oè les notations sont identiques ì celles du $\S 30$.

Le vecteur $\overrightarrow{e_{0}}$ etant fixé en $x$ et le vecteur $\vec{l}$ determiné par $S$, nous choisisson $\overrightarrow{e_{1}}$ tel qu'on puisse prendre $\vec{l}=\overrightarrow{e_{0}}+\overrightarrow{e_{1}}$. Pour un choix convenable de $\overrightarrow{e_{2}}$ dans le 2-plan $\left(\overrightarrow{e_{2}}, \overrightarrow{e_{3}}\right)$ la matrice $K_{v}^{u}$ a la forme $(30-9)$. Quant à la matrice $\left(\nabla_{\vartheta} F_{0}^{u_{0}}\right)$ elle a' d'après les considerations du $\S 9$, tous ses éléments nul pour $v \neq 1$ et d'aprés $(7,3)$ :

$$
\left[\nabla_{1} F^{1}{ }_{0}\right]=0 .
$$

Nous poson: $\left[\nabla_{1} F^{2}{ }_{0}\right]=\mu \quad\left[\nabla_{1} F^{3}{ }_{0}\right]=\vee$ et $(31.8)$ peut etre explicité par les relations:

$$
\left[\frac{d^{2} v^{1}}{d s^{2}}\right]=0 \quad\left[\frac{d^{2} v^{2}}{d s^{2}}\right]=\sigma v^{2}+\not k \mu v^{1} \quad\left[\frac{d^{2} v^{3}}{d s^{3}}\right]=-\sigma v^{3}+k v v^{1}
$$

qui donnent les composantes de la discontinité en $x$ de l'accéleration relative.

La composante le long de $\overrightarrow{e_{1}}$ de cette discontinuité est toujours nulle. Si $\vec{v}$ est colineaire à $\overrightarrow{e_{1}}$ la discontinuité est d'origine purement électromagnétique. Si $\vec{v}$ est orthogonal à $\overrightarrow{e_{1}}$, la discontinuité est d'origine purement gravitationelle.

\section{Remarques sur le cas pentadimensionnel}

\section{Composantes du tenseur de courbure, du tenseur de Ricci et $d u$} tenseur d' Einstein.

Les résultats précédents nous conduisent à étudier les fronts d'onde et les discontinuités du tenseur de courbure dans le cadre des théories pentadimensionnelles, théorie de KaLUZa-KLein et théorie de JoRDan-Tiry.

Nous considérons une variété riemanienne $V_{5}$ satisfaisant aux bypothèses du $\$ 19$ et nous rappelons d'abord les expressions des composantes $R_{\alpha \beta}, \lambda_{\mu}$ $(\alpha \ldots$ voir indice grec $=0,1,2,3,4)$ en repères orthonormés adaptés du tenseur 
de courbure. Si $i, j, . .=1,2,3,4$, il vient:

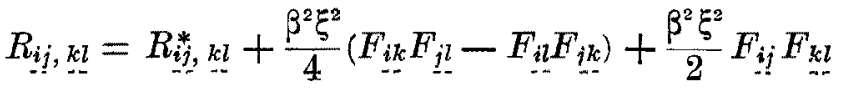

$$
\begin{aligned}
& R_{\underline{i}, \underline{k_{0}}}=\frac{\beta}{2}\left(\xi \nabla_{\underline{k}}^{*} F_{\underline{i j}}+2 \partial_{\underline{k}} \xi F_{\underline{i j}}-\partial_{\underline{i}} \xi F_{\underline{i k}}+\partial_{\underline{j}} \xi F_{\underline{i k}}\right) \\
& \left.R_{\underline{i 0,}, k_{0}}=\bar{\xi}^{-1} \nabla_{\underline{\underline{k}}}^{*} \partial_{\underline{i}} \xi\right)+\frac{\beta^{2} \xi^{2}}{4} F_{\underline{i r}} F_{\underline{\underline{k}}}
\end{aligned}
$$

où $F_{i j}$ a été identifié au tenseur champ électromagnétique, $\beta$ est une constante et où les éléments munis d'une * sont relatifs à la métrique quotient.

De (32-1) on déduit par contraction:

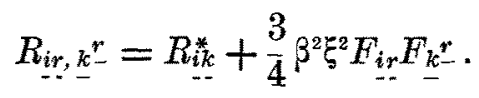

De (32-2), il vient:

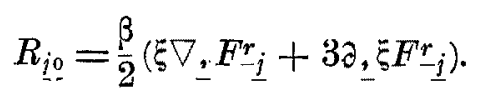

A partir de:

$$
R_{i k}=R_{i r, k^{r}}+R_{i 0, k^{0}}
$$

et de (32-4) et (32-5), on obtient, pour les composantes du tenseur de RIccr, les expressions suivantes:

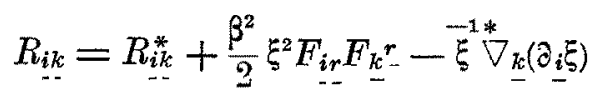

$$
R_{i 0}=\frac{\beta}{2} \dot{\xi}^{2 *} \nabla\left(\xi^{3} F_{-i}^{r}\right)
$$

Evaluons enfin les composantes du tenseur d'Ernsteis de $V_{5}$ :

$$
S_{\alpha \beta}=R_{\alpha \beta}-\frac{1}{2} \gamma_{\alpha \beta} R \text {. }
$$

De (32-6) il résulte:

$$
R_{i}^{i}=R^{*}+\frac{\beta^{2} \xi^{2}}{2} F_{r s} F^{r s}-\bar{\xi}^{-1} \Delta^{* \xi}
$$

et de (32-8)

$$
R_{\underline{0}}^{0}=-\bar{\xi}^{-1} \triangle^{* \xi}-F_{\underline{r s}} F^{r s} .
$$


Ainsi la courbure riemanienne scalaire $R$ a pour valeurs:

$$
R=R^{*}+\frac{\beta^{2} \xi^{2}}{4} F_{r s} F_{\stackrel{r}{r s}}-2 \bar{\xi}^{1} \Lambda^{*} \xi
$$

On obtient ainsi, pour le tenseur d'Enssterr, les composantes:

$$
\begin{aligned}
& S_{i k}=S_{i k}^{*}-\frac{\beta^{2} \xi^{2}}{2}\left(\frac{1}{4} g_{i k} F_{r s} F_{-r}^{r s}-F_{i r} F_{\underline{-}}^{r}\right)-\bar{\xi}^{1}\left(\bar{V}_{\underline{k}} \partial_{i} \xi-g_{i k} \triangle^{*} \xi\right) \\
& S_{i 0}=\beta \overline{2} \overline{V_{-}} \cdot\left(\xi^{3} F_{-i}^{r}\right) \\
& S_{00}=\frac{1}{2} R^{*}+\frac{3}{8} \beta^{2} \xi^{2} F_{\underline{r s}} F_{r s}^{r s} .
\end{aligned}
$$

Pour la théorie de JoRdan-TrRY, les équations de champ s'écrivent:

$$
S_{\alpha \beta}=\Theta_{\alpha \beta}
$$

où le tenseur second membre $\Theta_{\alpha \beta}$ décrit les sources du champ. Dans un domaine dépourru de sources, ce tenseur est nul (cas unitaire extérieur).

\section{3. Équations de champ dans la théorie de Kaluza-Klein.}

Dans la théorie de Kaldza-KLein $\xi=1$ et les formules précédentes relatives au tenseur de courbure prennent la forme:

$$
\begin{aligned}
& R_{i i, k l}=R_{i j, k l}^{*}+\frac{\beta^{2}}{4}\left(F_{i k-} F_{j l}-F_{i l} F_{i k}\right)+\frac{\beta^{2}}{2} F_{i j} F_{k l} \\
& R_{i j, k 0}=\frac{\beta}{2} \underline{V}_{\underline{k}} F_{i \underline{j}} \\
& R_{i \underline{0_{0}, k_{0}}}={ }_{4}^{\beta^{2}} F_{i r} F_{\underline{\underline{k}}} \text {. }
\end{aligned}
$$

Celles relatives au tenseur de RrCor s'écrivent:

$$
\begin{aligned}
& R_{i k}=R_{i \underline{k}}^{*}+\frac{\beta^{2}}{2} F_{i r} F_{k-} \\
& R_{i 0}=\frac{\beta}{2} \bar{V}_{\underline{-}}^{*} F_{-\underline{i}}^{r} \\
& R_{00}=\frac{\beta^{2}}{4} F_{* s} F^{r s}
\end{aligned}
$$


et celles relatives an tenseur d'EINSTEn:

$$
\begin{aligned}
& S_{i k}=S_{i k}^{*}-\frac{\beta^{2}}{2}\left(\frac{1}{4} g_{i k} F_{* s} F_{-s}^{r s}\right) \\
& S_{\underline{i 0}}=\frac{\beta}{2} \frac{*}{\underline{r}}_{\underline{r}} F^{r}{ }_{\underline{i}} \\
& S_{00}=\frac{1}{2} R^{*}+\frac{3}{8} \beta^{2} F_{z s} F^{r s} .
\end{aligned}
$$

Les quatorze équations au champ s'écrivent alors:

$$
S_{i k} \equiv R_{i k}-\frac{1}{2} g_{i k} R=\Theta_{i k}
$$

et

$$
S_{i 0} \equiv R_{i 0}=\Theta_{i 0}
$$

où les $\Theta$ décrivont les sources du champ. De (33-10) on déduira par contraction:

$$
g_{--}^{i k} R_{i k}-2 R=\Theta \quad\left(\Theta=\mathrm{g}_{.}^{i k} \Theta_{i k}\right)
$$

soit:

$$
R+R_{00}-2 R=\Theta
$$

Il en résulte:

$$
R=R_{00}-\Theta
$$

et (33-10) peut être mis sous la forme:

$$
R_{i k}=\frac{\beta^{2}}{8} g_{i k} F_{r s} F_{?-}^{r s}+\left(\Theta_{i k}-\frac{1}{2} g_{i k} \Theta\right)
$$

\section{Discontinuités a u tenseur de courbure de $V_{5}$.}

La métrique $\gamma_{\alpha \beta}$ de $V_{5}$ et le générateur infinitésimal $\vec{\xi}$ du groupe d'iso. métries sont supposés de classe $\left(C^{1}, C^{3}\right.$ par morceaux). Il en résulte que la métrique quotient et le scalaire $\xi$ sont anssi $\left(C^{1}, C^{2}\right.$ par morceau $\left.\mathrm{x}\right)$ alors que la tenseur $F_{i j}$ est continu et à dérivêes premières discontinues.

Plaçons-nous dans un domaine de $V_{5}$ où les seconds membres des équations de champ sont continus et étudions les discontinuités du tenseur de courbure de $V_{5}$ à la traversée d'une hypersurface $S$ engendrée par des trajectoires du groupe d'isométries. Eu coordonnées locales adaptées, l' equa. tion de $S$ est donc de la forme $f\left(x^{i}\right)=0$ et, dans ces coordonnées, tout 
vecteur $\vec{l}$ colinéaire au gradient de $f$ satisfait $l_{0}=0$, e'est-à-dire est orthogonal an recteur tangent à la trajectoire da groupe d'isométries. En repère orthonormé adapté $l_{0}=0$.

Dans les hypothèses faites, en théorie de JuRdan-THrRY, on a, à la traversée de $S$, en vertu de (18-4)

$$
\left[R_{\alpha \beta}\right]=0 .
$$

Cette relation est encore vérifiée dans les mêmes hypothèses, en theorie de KALUZA-KLEIN en verta de (33-12), (33-11) et (33-6).

D'après l'étude générale faite an $\$ 20, \vec{l}$ est de longueur nulle et le tenseur $\left[R_{\left.\alpha \beta, \lambda_{\mu}\right]}\right]$ satisfait aux relations:

$$
\begin{gathered}
l_{\alpha[}\left[R_{\beta \mu, \lambda_{\mu}}\right]+l_{\beta}\left[R_{\left.\gamma \alpha, \lambda_{\mu}\right]}\right]+l_{\gamma[}\left[R_{\alpha \beta, \lambda_{\mu} \mu}\right]=0 \\
l^{\alpha}\left[R_{\alpha \beta, \lambda_{\mu}}\right]=0
\end{gathered}
$$

en un point $x$ de $S$.

En ce point, considérons le vecteur normé $\overrightarrow{e_{0}}$ colinéaire à $\vec{\xi}$ et donnonsnous un vecteur unitaire $\overrightarrow{e_{4}} \overrightarrow{e_{4}^{*}}=1$ ) astreint senlement à être orthogonal a $\overrightarrow{e_{0}}$. Les vecteurs $\overrightarrow{e_{0}}$ et $\vec{l}$ definissent un 2-plan orthogonal à $\vec{e}$; dans ce 2-plan soit $\overrightarrow{e_{1}}$ le vecteur normé orthogonal à $\overrightarrow{e_{4}}$ tel qu'on puisse prendre:

$$
\vec{l}=\overrightarrow{e_{1}}+\overrightarrow{e_{1}} \text {. }
$$

On peut completer par deux vecteurs $\overrightarrow{e_{2}}, \overrightarrow{e_{3}}$ da 2-plan orthogonal au 3-plan défini par $\left.\overrightarrow{\left(\vec{e}_{0}\right.}, \overrightarrow{e_{1}}, \overrightarrow{e_{4}}\right)$ de façon à obtenir en $x$ un repère $\left(\overrightarrow{e_{\alpha}}\right)$ orthonomé adapté tel que (34-4) soit satisfaite. C'est relativement à un tel repère que nous raisonnerons dans la suite.

On notera que comme $l_{0}=0$, les $l_{i}$ définissent au point correspondant de la variété-quotient un vecteur de longueur nulle.

\section{Matrice représentative d'un tenseur $H_{\alpha \beta, \lambda_{\mu}}$ en $x$.}

Soit $H_{\alpha \beta, \lambda_{\mu}} \neq 0$ un tenseur jouissant des mémes propriétés de symétrie que le tenseur de courbnre et satisfaisant à (21-2) et $(21.3)$ pour un vecteur $\vec{l}$ orthogonal $\grave{a} e_{0}$. Ce vecteur est nécessairement de longueur nulle et nous pouvons adopter en $x$ le repère $\left(\vec{e}_{\alpha}\right)$ introduit au paragraphe précédent. $(21.2)$ et $(21.3)$ sont alors traduites par les relations:

$$
H_{\alpha \beta, \lambda \mu}=a_{00} \varphi_{\alpha \beta}^{(0)} \varphi_{, \mu}^{(0)}+\underset{A}{\Sigma} a_{0 A}\left(p_{\alpha \beta}^{0} \varphi_{\lambda \mu}^{(A)}+\varphi_{\alpha \beta}^{(A)} f_{\lambda \mu}^{(0)}\right)+\underset{A, B}{\Sigma} a_{A B} \varphi_{\alpha \beta}^{(A)} \varphi_{\lambda, \mu}^{(B)}
$$

où les tenseurs $\varphi$ sont les produits extérieurs de $\vec{l}$ avec les vecteurs $\overrightarrow{n^{0}}=\overrightarrow{e_{0}}$, $\overrightarrow{n^{(1)}}=\overrightarrow{e_{2}} \overrightarrow{n^{(2)}}=\overrightarrow{e_{3}}$ et où les indices $A, B$ prennent les valeurs $1,2$. 
En posant:

$$
\left\{\begin{array}{lll}
\overrightarrow{e_{2}} \wedge \overrightarrow{e_{3}}=\tau_{2} & \overrightarrow{e_{1}} \wedge \overrightarrow{e_{4}}=\tau_{4} & \overrightarrow{e_{1}} \wedge \overrightarrow{e_{0}}=\tau_{7} \\
\overrightarrow{e_{3}} \wedge \overrightarrow{e_{1}}=\tau_{2} & \overrightarrow{e_{2}} \wedge \overrightarrow{e_{4}}=\tau_{5} & \overrightarrow{e_{2}} \wedge \overrightarrow{e_{0}}=\tau_{8} \\
\overrightarrow{e_{1}} \wedge \overrightarrow{e_{2}}=\tau_{3} & \overrightarrow{e_{3}} \wedge \overrightarrow{e_{4}}=\tau_{8} & \overrightarrow{e_{3}} \wedge \overrightarrow{e_{0}}=\tau_{8} \\
& \overrightarrow{e_{4}} \wedge \overrightarrow{e_{0}}=\tau_{10}
\end{array}\right.
$$

on est conduit, comme au $\$ 21$, à représenter $H_{\alpha \beta, \lambda \mu}$ par une matrice $\left(H_{I J}\right)$ symétrique $(I, J=1, \ldots .10) ;(35.1)$, compte-tenu des composantes des tenseurs $\varphi$, conduit pour la matrice $\left(H_{I J}\right)$ à la forme:

\begin{tabular}{c|cccccc|cccc}
\multicolumn{1}{l}{} & 1 & 2 & 3 & 4 & 5 & 6 & 7 & 8 & 9 & 10 \\
\hline 1 & 0 & 0 & 0 & 0 & 0 & 0 & 0 & 0 & 0 & 0 \\
2 & 0 & $a_{22}$ & $-a_{12}$ & 0 & $-a_{12}$ & $-a_{22}$ & $-a_{02}$ & 0 & 0 & $a_{02}$ \\
3 & 0 & $-a_{12}$ & $a_{12}$ & 0 & $a_{11}$ & $a_{12}$ & $a_{01}$ & 0 & 0 & $-a_{01}$ \\
4 & 0 & 0 & 0 & 0 & 0 & 0 & 0 & 0 & 0 & 0 \\
5 & 0 & $a_{12}$ & $a_{11}$ & 0 & $a_{11}$ & $a_{12}$ & $a_{01}$ & 0 & 0 & $-a_{01}$ \\
6 & 0 & $-a_{22}$ & $a_{12}$ & 0 & $a_{12}$ & $a_{22}$ & $a_{02}$ & 0 & 0 & $-a_{02}$ \\
& & & & & & & & & & \\
7 & 0 & $-a_{02}$ & $a_{01}$ & 0 & $a_{01}$ & $a_{02}$ & $a_{00}$ & 0 & 0 & $-a_{00}$ \\
8 & 0 & 0 & 0 & 0 & 0 & 0 & 0 & 0 & 0 & 0 \\
9 & 0 & 0 & 0 & 0 & 0 & 0 & 0 & 0 & 0 & 0 \\
10 & 0 & $a_{02}$ & $-a_{01}$ & 0 & $-a_{01}$ & $-a_{02}$ & $a_{00}$ & 0 & 0 & $a_{00}$
\end{tabular}

On sait que nécessairement:

$$
H_{\alpha \beta}=\tau l_{\alpha} l_{\beta}
$$

En contractant $(35.1)$, il vient:

soit :

$$
H_{\alpha \beta}=-\left(a_{00}+a_{11}+a_{22}\right) l_{\alpha} l_{\beta}
$$

$$
\tau=-\left(a_{00}+a_{11}+a_{22}\right)
$$

Ainsi pour que $H_{\alpha \beta}=0$, il faut et il suffit que $a_{00}+a_{11}+a_{22}=0$. 
A. Lichnerowicz: Ondes et radiations électromagnétiques, etc.

\section{Matrice des discontinuités du tenseur de courbure dans les théories pentadimensionnelles.}

Dans le cas du tenseur $\left[R_{\alpha \beta, \lambda \mu}\right]$, on a $\left[R_{\alpha \beta}\right]=0$ et par suite $a_{00}+a_{11}+a_{22}=0$. Pour la théorie de KALUZA-KLEIN on a en outre, manifestement $\left[R_{i 0},{ }_{n 0}\right]=0$ et par suite $a_{00}=a_{11}+a_{22}=0$. Ainsi la matrice $\left(\left[R_{I J}\right]\right)$ a la forme $(35.2)$ soit avec $a_{00}+a_{11}+a_{22}=0$ (theorie de JORDAN-THIRY) soit avec $a_{00}=a_{11}+a_{22}=0$ (théorie de KaIdZA-KLEIN).

On retrouve aisément cette forme réduite à partir des formules:

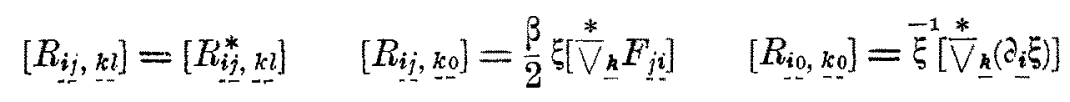

et des résultats antérieurs. Notons d'abord que d'après la condition de HA. DAMARD, il existe un scalaire $a_{00}$ en $x$ tel que:

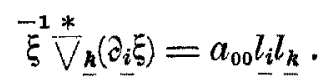

Le repère orthonormé adapté étant choisi de façon que $\vec{l}=\overrightarrow{e_{4}}+\overrightarrow{e_{1}}$, il résulte de (36.1) que la sous-matrice de $\left(\left[R_{I J}\right]\right)$ correspondant à $7 \leq I, J \leq 10$, a la forme lisible sur (35.2). De plus de (32.6) on déduit:

$$
\left[R_{i \underline{k}}^{*}\right]=\vec{\xi}^{1}\left[\ddot{V}_{\underline{k}}^{*}\left(\partial_{i} \xi\right)\right]=a_{00} l_{\underline{\underline{k}}} l_{\underline{k}}
$$

Ainsi $\left[R_{i j}^{*}, k l\right]$ qui satisfait

$$
l_{\underline{k}}\left[R_{i j}^{*}, k l\right]+l_{[}\left[R_{i k,-2}^{*}\right]+l_{\underline{j}}\left[R_{k i, k l}^{*}\right]=0
$$

et (36.2) reprêsentè, par la sous-matrice $\left(\left[R_{I J}\right]\right)(1 \leq I, J \leq 6)$ correspondant à (35.2) avec $a_{00}=-\left(a_{11}+a_{22}\right)$. Enfin de

$$
\frac{\beta}{2} \xi\left[\stackrel{*}{V}_{\underline{k}} F_{-}^{i j}\right]=\varphi_{i} l_{\underline{k}}
$$

où $\varphi_{i j}$ est singulière et de l'expression de cette forme pour $\vec{l}=\overrightarrow{e_{4}}+\overrightarrow{e_{1}}$, on déduit la sous-matrice correspondant a $1 \leq I \leq 6,7 \leq J \leq 10$.

\section{Radiation en théorie de Kaluza-Klein.}

10) Plaçons-nous dans le cadre de la théorie de Kaldza-Klein, la varieté $V_{5}$ étant rapportée à des repères orthonormés adaptés. Pour simplifier les notations, nous supprimons dans la suite le soulignage des indices. Suppo. 
sons que la métrique d'espace-temps $d s^{2}$ corresponde à un état de radiation totale pure, le champ électromagnétique représentant lui-même un état de radiation électromagnétique de même vecteur fondamental isotrope. Nous introduisons le vecteur isotrope de $V_{5}$ orthogonal à $\vec{\xi}$ et se projetant sur $V_{4}$ selon le vecteur précédent.

Sous nos hypothèses, nous avons d'une part:
(a) $S l_{h} R_{i j, k l}^{*}=0$
(b) $l^{i} R_{i j, k l}^{*}=0$

où $S$ désigne ici la sommation après permutation circulaire sur les indices $h, i, j$ et d'autre part:

$$
\begin{array}{ll}
\text { (a) } S l_{h} F_{i j}=0 & \text { (b) } l^{i} F_{i j}=0 .
\end{array}
$$

La formule (33.1) nous conduit à etudier, pour une 2-forme $F$ singuliere, le tenseur

$$
S l_{h}\left(F_{i k} F_{j l}-F_{i l} F_{j k}\right)
$$

Il vient:

$$
S l_{h}\left(F_{i k} F_{j l}-F_{i l} F_{j k}\right)=-S l_{h}\left(F_{i k} F_{l j}+F_{i l} F_{j k}\right) .
$$

Or $F$ étant une 2-forme singulière est un produit extérieur et:

$$
F_{i k} F_{l j}+F_{i l} F_{j k_{i}}+F_{i j} F_{k l}=0 .
$$

Ainsi :

$$
S l_{h}\left(F_{i h} F_{j l}-F_{i l} F_{j k}\right)=\left(S l_{h} F_{i j}\right) F_{k l} .
$$

Nous voyons done que, pour une 2-forme $F$ singulière,

$$
S l_{h}\left(F_{i k} F_{i l}-F_{i l} F_{j k}\right)=0 \quad l^{i}\left(F_{i k} F_{j b}-F_{i l} F_{j k}\right)=0 .
$$

Les hypothèses $(37.1)$ et (37.2) entrânent ainsi, à partir de (37-3),

$$
\begin{array}{ll}
\text { (a) } S l_{h} R_{i j, k l}=0 & \text { (b) } l^{i} R_{i j, k l}=0 .
\end{array}
$$

D'autre-part de (33-3), on déduit:

$$
l_{h} R_{i 0, k_{0}}-l_{i} R_{h_{0}, k_{0}}=\frac{\beta^{2}}{4}\left(l_{h} F_{i r}-l_{i} F_{h r}\right) F_{k}{ }^{r}
$$

soit, d'après $(37.2)_{\mathrm{a}}$ :

$$
l_{h} R_{i 0_{0}, k_{0}}-l_{i} R_{h_{0}, k_{0}}=\frac{\beta^{2}}{4} l_{r} F_{i h} F_{k}^{r}
$$

On a ainsi d'après $(37-2)_{\mathrm{b}}$ :

$$
\text { (a) } l_{h} R_{i 0, k_{0}}+l_{i} R_{0 h, k_{0}}=0 \quad l^{i} R_{i 0, k_{0}}=0 .
$$


$2^{\circ}$ ) Introdnsons le tensenr de $V_{5}$ ayant mème type de symétrie que le tenseur de courbure et dólini, relativement aux repères orthonomés adaptes, par:

$$
P_{i j, k l}=R_{i j, k l} \quad P_{i j, k 0}=0 \quad P_{i j, k 0}=-R_{i n, k_{0}}
$$

oǹ le signe-figure pour des raisons de signature. Le tenseur $P_{\alpha \beta}$. contracté de $P_{\alpha \beta, \gamma \delta}$ vérifie:

$$
P_{i 0}=0 \quad P_{00}=-R_{00}
$$

$l_{0}$ ctant nul, les relations $(37-4)$ et $(37.5)$ peuvent s'exprimer par:

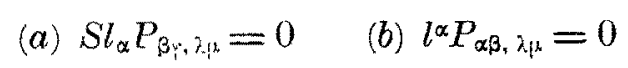

où le vecteur $l_{\alpha}$, est orthogonal à $\xi_{\alpha}$.

$\left.3^{\circ}\right)$ Inversement supposons que le tenseur $P_{\alpha \beta, \gamma)}$ défini en repèr's orthonormís adaptés par (37-6) soit tel qu'il existe un vecteur $l_{\alpha}$ orthogonal it $\xi_{\alpha}$ pour lequel les relations $(37-8)$ soient satisfaites.

$P_{\alpha \beta}$ est proportionnel is $l_{\alpha} l_{\beta}$. Comme $l_{0}=0$, on a $P_{00}=0$, soit $R_{00}=0$ et diapres (33-6)

$$
F_{r s} F^{r s}=0
$$

De plus, daprès $(37-5)_{h}$, :

$$
l^{i} K_{i}, F_{k}{ }^{r}=0
$$

$l^{i}$ eit ainsi vecteur propre, avec la valeur propre 0 , du tenseur de MaxweiL, de $F$ ot la 2 -forme $F$ est singuliere. De (37-4) on tire, compte-tenu de (37-3)

$$
S l_{h} R_{i j, k l}^{*}=0 \quad l^{i} R_{i, k l}^{*}=0
$$

et la métrique $d s^{2}$ définit un état de radiation totale pure.

Supposons que les équations de champ (33.12) soient satisfaites en l'absence de sources $\left(\Theta_{i k}=0\right)$. De $(37.9)$ on déduit:

$$
R_{i k}=0
$$

La 2-forme $F$ thant singuliere:

$$
\frac{\beta^{2}}{2} F_{i} \cdot F_{k}{ }^{r}=-\tau l_{i} l_{k}
$$

et d'après (33.4)

$$
R_{i k}^{*}=\tau l_{i} l_{k}
$$

ce qui explique l'expression d'état de «radiation totale pure». 
38. Expression intrinsèque du tensenr $P_{\alpha \beta, \gamma[\mu}$.

Il est possible d'oxprimer intrinséquement le tenseur $P_{\alpha \beta . \gamma \delta}$ à partir du tenseur de courbure de $V_{5}$ et du générateur $\vec{\xi}$ dn groupe d'isométries. A cet effet introduisons le vectear $\vec{u}$ normé $\left.\overrightarrow{u^{2}}=-1\right)$ colinéaire à $\vec{\xi}$ et étudions le tenseur de $V_{5}$ défini par:

$$
t_{\alpha \beta}^{\lambda \mu}=g_{\alpha}^{\lambda} g_{\beta}^{\mu}+g_{\alpha}^{\lambda} u_{\beta} u^{\mu}+g_{\beta}^{\mu} u_{\alpha} u_{\lambda}
$$

et qui satisfait:

$$
t_{\alpha \beta}^{\lambda \mu}=t_{\beta \boldsymbol{\alpha}}^{\mu \lambda}
$$

En repères orthonormés adaptés, on $a u^{\lambda}=g_{0}^{2}, u_{i}=0, u_{0}=-1$. On en déduit:

$$
\begin{aligned}
& t_{i j}^{2 \mu}=g_{i}^{\lambda} g_{j}^{n} \\
& t_{0 i}^{\lambda}=g_{0}^{\lambda} g_{i}^{l}-u^{\lambda} \cdot g_{i}^{\mu}=0 \\
& t_{00}^{\lambda \mu}=g_{0}^{\lambda} g_{0}^{\prime 2}-g_{0}^{\lambda} g_{0}^{\mu}-g_{0}^{\lambda} g_{0}^{\mu}=-g_{0}^{\lambda} g_{0}^{\mu} \text {. }
\end{aligned}
$$

A partir de (38.2), (38.3), (38-4), il est aisé de vérifier que l'on a:

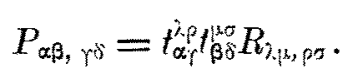

En effet d'après (38-2)

$$
P_{i j, k l}=R_{i j, k l}
$$

D'après (38.3)

$$
P_{i j, k 0}=0
$$

et d'après $(38.2)$ et $(38-4)$ :

$$
P_{i 0, k 0}=-R_{i 0, k_{0}}
$$

ce qui est en accord avec les relations du $\$ 37$.

38. Radiation en théorie de Jordan-Thiry.

$\left.1^{\circ}\right)$ Sur $V_{4}$ munie de la métrique-quotient $d s^{2}$, considerons le champ scalaire $\xi$ et supposons qu'il existe un vecteur $l^{i}$ tel que:

$$
\begin{array}{ll}
\text { (a) } l_{i} \stackrel{*}{\nabla}_{k} \partial_{j} \xi-l_{j} \vec{\nabla}_{k} \partial_{i} \xi=0 & \text { (b) } l^{i} \nabla_{k}^{*} \partial_{i} \xi=0
\end{array}
$$

où $\stackrel{\vee}{*}_{k} \partial_{i} \xi$ est $\neq 0$. Si $\vec{v}$ est un vecteur arbitraire en $x \in V_{4}$, on tire de $(39.1)_{a}$ par produit contracté par $v^{k}$ :

$$
v^{k} \stackrel{*}{\nabla}_{k} \partial_{i} \xi=\lambda \overrightarrow{(v)} l_{i}
$$


où $\lambda(\vec{v})$ est une forme linéaire en $\vec{v}$. Il en résulte $\vec{\nabla}_{k}^{*} \partial_{i} \xi=\lambda_{k} l_{i}$ et par raison de symétrie:

$$
\stackrel{*}{\nabla}_{k} \partial_{i} \xi=\tau l_{i} l_{k}
$$

où $\tau$ est un scalaire. Ainsi pour que les relations (39.1) soient satisfaites, il faut et il suffit que l'on ait (39.2) où $l_{i}$ est un vecteur isotrope. En particulier:

$$
\triangle * \xi \equiv 0 \text {. }
$$

Lorsque les hypothèses (39-1) sont satisfaites, nous dirons que le champ scalaire $\xi$ correspond à un etat de radiation pure.

$\left.2^{\circ}\right)$ Dans le cadre de la théorie de Jordan-ThrRy, supposons que la métrique quotient $d s^{2}$ (qui diffère ici de la métrique d'univers $\tilde{d s^{2}}=\xi d s^{2}$ ), la forme $F$ et le scalaire $\xi$ satisfassent aux conditions de radiation pour le mème vecteur fondamental isotrope. En repères orthonormés adaptés, on a (39-2),

$$
S l_{h} R_{i j, k l}^{*}=0 \quad l^{i} R_{i j, k l}^{*}=0
$$

et

$$
S l_{h} F_{i j}=0 \quad l^{i} F_{i j}=0 .
$$

Ces relations entraĩnent, en vertu de (32-1) et (32.3), les relations :

$$
S l_{\alpha} P_{\beta \gamma, \lambda \mu}=0 \quad l^{\alpha} P_{\alpha \beta, \lambda \mu}=0
$$

où $P_{\alpha \beta, \lambda_{j}}$ est encore défini par (38-5).

40. Construction d'un exemple de radiation pure avec champ electromagnétique.

Proposons nous de construire un exemple de radiation totale pure satisfaisant aux équations de la théorie de Kaloza.KLEIN en l'absence de sources. Sur l'espace-temps $V_{4}$, nous devons ainsi obtenir un champ gravitationnel verifiant $(37-1)$ et (37.2) et un champ électromagnétique singulier satisfaisant aux équations d'EINSTErn-NLXweLL de la relativité générale.

$\left.1^{\circ}\right)$ Considérons un domaine $D$ de l'espace-temps mnni de la métrique (27.2) qui peut s'écrire avec de nouvelles notations:

$$
d s^{2}=e^{2} \psi\left[\left(d x^{4}\right)^{2}-\left(d x^{1}\right)^{2}\right]-\left[\xi^{2}\left(d x^{2}\right)^{2}+\eta^{2}\left(d x^{3}\right)^{2}\right]=g_{i j} d x^{i} d x^{j}
$$

où $\psi . \xi>0, \eta>0$ sont trois fonctions de la seule variable:

$$
u=x^{4}-x^{1} .
$$

Introduisons un potentiel-vecteur $\varphi_{i}$ défini en condonnées $\left(x^{k}\right)$ par:

$$
\varphi_{1}=\varphi_{2}=\varphi_{4}=0 \quad \varphi_{3}=\varphi(u) .
$$


A u potentiel-vecteur correspond une 2-forme $F$ dont les seules composantes non nulles sont:

$$
F_{34}=-\partial_{4} \varphi=-\varphi^{\prime} \quad F_{31}=-\partial_{1} \varphi=\varphi^{\prime} .
$$

Le vecteur $l_{i}$, gradient de $u$, dont les composantes covariantes sont :

satisfait

$$
l_{1}=-1 \quad l_{2}=l_{3}=0 \quad l_{4}=1
$$

$$
S l_{h} F_{i j}=0
$$

où $S$ indique la sommation après permatation circulaire. Cette relation est en effet satisfaite pour $h=4$ et soit $i=2, j=3$, soit $i=3, j=1$, soit $i=1$, $j=2$. D'autre part $l^{i}$ admettaut les composantes contravariantes:

on a:

$$
l^{4}=l^{1}=e^{-2 \psi} \quad l^{2}=l^{3}=0
$$

$$
l^{i} F_{i j}=0 .
$$

Ainsi la forme $F$ est singulière et admet le vectenr fondamental isotrope $l_{i}$.

$2^{\circ)}$ Evaluons $\stackrel{*}{\vee} l_{i}$, où $\stackrel{*}{\vee} j$ est le symbole de dérivation covariante dans le métrique (40.1). On a d'abord.

Or :

$$
\stackrel{*}{\vee}_{2} l_{i}=-\stackrel{*}{\Gamma}_{i 2}^{4}+\stackrel{*}{\Gamma}_{i 2}^{1} \text {. }
$$

$$
{\stackrel{*}{\Gamma_{i 2}^{4}}}_{i}^{4} g^{44}[i 2,4]=\frac{1}{2} e^{-2} \psi g_{i 2}^{\prime} \quad{\stackrel{*}{\Gamma_{i 2}}}_{i}^{1}=g^{11}[i 2,1]=\frac{1}{2} e^{-2 * g_{i 2}^{\prime}} .
$$

On obtient ainsi :

et de même:

$$
{\stackrel{*}{V_{2}}}_{l} l_{i}=\stackrel{*}{\nabla}_{i} l_{2}=0
$$

D'autre part:

$$
\stackrel{*}{V}_{3} l_{i}=\stackrel{*}{V} l_{3}=0 \text {. }
$$

$$
\stackrel{*}{\nabla_{1}} l_{1}=-\Gamma_{11}^{4}+\stackrel{*}{\Gamma}_{11}^{1} .
$$

Or d'après les résultats du $\$ 27$ :

Il en résulte:

$$
\stackrel{*}{\Gamma}_{11}^{4}=\psi^{\prime} \quad \stackrel{*}{\Gamma}_{11}^{1}=-\psi^{\prime} .
$$

et de même:

$$
\stackrel{*}{V_{1}} l_{1}=-2 \Psi^{\prime}
$$

$$
\stackrel{*}{V_{4}} l_{1}=\stackrel{*}{V_{1}} l_{4}=2 \psi^{\prime} \quad{ }^{*} l_{4}=-2 \psi^{\prime} .
$$

Nous avons ainsi établi que:

$$
\stackrel{*}{V_{j}} l_{i}=-2 \psi^{\prime} l_{i} l_{j}
$$


De la relation (40.2) on déduit par dérivation:

$$
S l_{h} \stackrel{*}{V}_{k} F_{i j}+S \bar{V}_{k} l_{h} \cdot F_{i j}=0
$$

soit, en vertu de (40.4),

$$
S l_{h} \stackrel{*}{\vee}{ }_{k} F_{i j}-2 \psi^{\prime} l_{k} S l_{h} F_{i j}=0
$$

c'est à-dire :

$$
S l_{h} \bar{\vee}_{k} F_{i j}=0 \text {. }
$$

De même, par dérivation de (40-3), on obtient :

Soit, en vertu de (40.4),

$$
l^{i} \stackrel{*}{\vee} k_{k} F_{i j}+\stackrel{*}{\vee}_{k} l^{i} F_{i j}=0 \text {. }
$$

$$
l^{i} \stackrel{*}{\vee}_{k} F_{i j}=0 \text {. }
$$

$3^{\circ}$ ) Considérons, sur la variété $V_{5}$ de la théorie de KaLUZa-KLEIN, la métrique :

$$
d \sigma^{2}=e^{2} \psi\left[\left(d x^{4}\right)^{2}-\left(d x^{1}\right)^{2}\right]-\left[\xi^{2}\left(d x^{2}\right)^{2}+\eta^{2}\left(d x^{3}\right)^{2}\right]-\left(d x^{0}-\varphi d x^{3}\right)^{2}=\gamma_{\alpha \beta} d x^{\alpha} d x^{\beta} .
$$

La métrique-quotient par le groupe d'isométries $x^{0}-x^{0}+$ const. coinncide avec $d s^{2}$ et les relations (33-1), 33.2), (33-3) s'appliquent avec la constante $\beta=1$, en repères orthonormés adaptés. La forme $F$ étant singulière, on $a$ :

$$
S l_{\underline{h}}\left(F_{i k} F_{\underline{j l}}-F_{i l} F_{j k}\right)=0 \quad l_{-}^{i}\left(F_{i k} F_{j l}-F_{\underline{i l}} F_{\underline{j}}\right)=0 .
$$

D'autre part d'après les résultats du $\$ 27$ :

$$
S l_{h} R_{i j, k l}^{*}=0 \quad l^{i} R_{i j, k l}^{*}=0
$$

Ainsi l'on a :

$$
S l_{h} R_{i, k l}=0 \quad l \underline{i} R_{i j, k l}=0 .
$$

Les relations $(40-5)$ et (40-6) expriment que:

$$
S l_{h} R_{i j, k_{0}}=0 \quad l_{-}^{i} R_{i j, k_{0}}=0:
$$

Enfin du caractère singulier de $F^{\prime}$ et du $\$ 37,1^{\circ}$ il résulte

$$
l_{h} R_{i 0, k_{0}}+l_{i} R_{0 h, k_{0}}=0 \quad l \underline{i} R_{i 0, k_{0}}=0 .
$$

Ainsi le vecteur $l_{\alpha}$, de composantes $l_{\underline{\xi}}$, orthogonal à $\underline{\xi}_{\alpha}$, satisfait:

$$
S l_{\alpha} R_{\beta \gamma, \lambda_{\mu}}=0 \quad l^{\alpha} R_{\alpha \beta, \lambda_{\mu}}=0 .
$$

On sait $q u^{\prime}$ il en résulte:

$$
R_{\alpha \beta}=\tau l_{\alpha} l_{\beta}
$$


$\left.4^{\circ}\right)$ D' après (40-12), $R_{i 0}=0$ et les équations de MAXwELL sont satisfaites pour $F$. Pour vérifier les équations de champ (33.12) en l'absence, de sources, soit $R_{i k}=0$, il nous suffit d'après (40-12) de vérifies $R_{44}=0$, soit d'après (33-4):

$$
R_{\underline{4}}^{*}+\frac{1}{2} F_{\underline{4}-\underline{\underline{4}}} F_{\underline{4}}^{r}=0
$$

ou en coordonnées $\left(\boldsymbol{x}^{i}\right)$

$$
R_{44}^{*}+\frac{1}{2} F_{4 r} F_{4}^{r}=0
$$

Or d'après les résultats du $\S 27$ :

D'autre part :

$$
R_{* 4}^{*}=-\vec{\xi}^{-1}\left(\xi^{\prime \prime}-2 \psi^{\prime} \xi^{\prime}\right)-\bar{\eta}^{-1}\left(\eta^{\prime \prime}-2 \psi^{\prime} \eta^{\prime}\right)
$$

$$
F_{4 r} F_{4}{ }^{r}=F_{43} F_{4}^{3}=-\eta^{-2} \varphi^{\prime 2}
$$

$1 l$ en résulte que les fonctions $\xi, \eta, \psi, \varphi$ doiveut étre liées par la relation:

$$
\bar{\xi}^{-1}\left(\xi^{\prime \prime}-2 \psi^{\prime} \xi^{\prime}\right)+\bar{\eta}^{-2}\left(\eta^{\prime \prime}-2 \psi^{\prime} \eta^{\prime}\right)+\frac{1}{2} \bar{\eta}^{-1} \varphi^{\prime 2}=0
$$

En nous limitant à $u>0$ et posant $\xi \eta=u^{2}, \eta / \xi=e^{2 \beta},(40-13)$ peut étre mis sous la forme:

$$
2 \psi^{\prime}=u \beta^{12}+\frac{1}{4 u} e^{-2 \beta} \varphi^{\prime 2}
$$

Si $\beta$ et $\varphi$ sont des fonctions données pour $u>0$, (40-10) permet dans le mềme domaine de déterminer $\psi$ par quadrature.

Pour la partie $\left(R^{k}\right)^{+}(u>0)$ de l'espace te:aps $R^{t}$ défini pas les $x^{i}$, effectuons sur la métrique $d s^{2}$ le changement de variables (28-7). Sur $\left(R^{4}\right)^{+}$on obtient, compte-tenu de (40.14), l'expression suivante de la métrique:

$$
\begin{gathered}
d s^{2}=d u d \bar{v}-\left(d \bar{y}^{2}+d \overline{z^{2}}\right)-2 \beta^{\prime}\left(\bar{y} d \bar{y}-\bar{z} d \bar{z}-\frac{\bar{y}^{2}-\bar{z}^{2}}{u} d u\right) d u-\beta^{\prime 2} u \bar{v} d u^{2}- \\
-\left(\bar{v}-\frac{\overline{y^{2}}+\overline{z^{2}}}{u}\right) \frac{1}{4 u} e^{-2 \beta} \varphi^{\prime 2} d u^{2}
\end{gathered}
$$

$u_{0}$ étant strictement positif, choisisson pour $\beta^{\prime}$ et $\varphi^{\prime}$ des fonctions de classe $C^{2}$ nulles pour $u \leq u_{0}$. On obtient ainsi une métrique euclidienne pour $u \leq u_{0}$, non enclidienne pour $u>u_{0}$, qui jointe à la forme $F$ introduite définit une solution $d u$ problème que nous nous étions posé. 


\section{Un processus de quantification de champs.}

\section{A. LE CHAMP ÉLECTROMAGNÉTIQUE.}

\section{Transformation de Fourier.}

En théorie quantique des champs, le champs électromagnétique $F_{\alpha \beta}$ se trouve généralement quantifié par recours au potentiel-vecteur et étude des transtormations de jauge correspondantes. Nous allons indiquer un processus de quantification directe du champ électromagnétique en relativité restreinte. Ce processus, qui est étroitement lié à la notion de radiation électromagnétique, pent être adapté, comme nous le verrons, à une quantification du champ gravitationnel.

Dans toute cette section, $V_{4}$ est l'espace-temps de la relativité restreinte supposé rapporté à des repères orthonormés $\left.\overrightarrow{e_{\alpha}}\right)(\alpha$, out indice grec $=0,1$, $2,3)$ La mélrique de $V_{4}$ rapporté à de tels repères sera désignée par

$$
d s^{2}=\eta_{\alpha \beta} d x^{\alpha} d x^{\beta}
$$

où le point $\vec{x} \in V_{4}$ admet les coordonnées $\left(x^{\alpha}\right)$. Considérons un champ électro. magnétique $F$ supposé satisfaire aux équation de Maxwell avec vecteur courant électrique identiquement nul, éest-à-dire à :

$$
S \partial_{\alpha} F_{\beta_{\gamma}}=0 \quad \partial_{\alpha} F^{\alpha_{\beta}}=0
$$

où $S$ désigne la sommation après permutation circulaire sur les trois indices.

Sous des hypothèses simples, le courant défini par $F$ est une transformée de Fourier sur l'espace-temps et l'on peat écrire en un sens évident (avec l'abus de notation usuel chez les physiciens)

$$
F_{\alpha \beta} \overrightarrow{(x)}=\frac{1}{(2 \pi)^{4}} \int U_{\alpha \beta(\vec{p})} e^{\vec{p}, \vec{x}} d \tau(\vec{p})
$$

où $\vec{p}$ décrit l'espace minkowskien et où $d \tau(\vec{p})$ est l'élément d'hypervolume correspondant:

$$
d \tau\left(\overrightarrow{p)}=d p^{0} \wedge d p^{1} \wedge d p^{2} \wedge d p^{3}\right.
$$

De (41.2) on déduit:

$$
S p_{\alpha} U_{\beta \gamma}=0 \quad p^{\alpha} U_{\alpha \beta}=0
$$


Par suite $p$ n'est différent de 0 que s'il est isotrope. $V_{4}$ étant rapporté à un repère $\left(\overrightarrow{e_{\alpha}}\right)$, soit $\vec{p}$ un vecteur arbitraire de l'espace-temps et posons

$$
\vec{p}=\vec{l}+\lambda \overrightarrow{e_{0}}
$$

où les composantes dn vecteur isotrope

$$
\vec{l}=l^{0} e_{0}+l^{u} \overrightarrow{e_{u}}
$$$$
(u=1,2,3)
$$

satisfont à

$$
\left(l^{0}\right)^{2}=\underset{u}{\Sigma}\left(l^{u}\right)^{2}
$$

D'après l'expression de $d \tau(\vec{p})$, on a:

où

$$
d \tau\left(\overrightarrow{p)}=l^{0} d \lambda \wedge d \overrightarrow{Q(\vec{e})}\right.
$$

$$
d \mathrm{Q}(\vec{l})=\frac{d l^{1} \wedge d l^{s} \wedge d l^{3}}{l^{0}}
$$

est l'élément de volume invariant du còne isotropie $C$. En introduisant une measure de Drrac de la variable $\lambda$, on pent mettre (41-3) sous la forme:

$$
F_{\alpha \beta} \overrightarrow{(x)}=\frac{1}{(2 \pi)} \int_{C} G_{\alpha \beta}(\vec{l}) e^{i \vec{r} \cdot \vec{x}} d Q(\vec{l}) .
$$

\section{Conditions de quantification.}

A la 2-forme $F$ à valeurs scalaires, substituons une 2-forme désignée par la même notation et à valenrs dans un espace vectoriel $M$ d'opérateurs de l'espace de Hilbart complexe. Nous ferons les hypothèses suivantes:

a) les valeurs de $F$ sont des opérateurs hermitiens

b) $F$ vérifie des équations (41-2).

Nous désignons par * le passage à un opérateur adjoint. La formule (41.4) est encore valable ici pourva que $G_{\alpha \beta}$ soit un tenseur à valeurs dans $M$. Dans cette formule, il résulte:

$$
F_{\alpha \beta}^{*}(\vec{x})=\frac{1}{(2 \pi)^{3}} \int G_{\alpha \beta}^{*}(\vec{l}) e^{-i \vec{l} \cdot \vec{x}} d \Omega(\vec{l})
$$

soit en changeant $\vec{l}$ en $-\vec{l}$

$$
F_{\alpha \beta}^{*}\left(\overrightarrow{x)}=\frac{1}{(2 \pi)^{3}} \int G_{\alpha \beta}^{*}\left(-\overrightarrow{l)} e^{i \vec{l} \cdot \vec{x}} d \mathbf{Q}(\vec{l}) .\right.\right.
$$

Du caractère hermitien des valenrs de $F$, on déduit ainsi :

$$
G_{\alpha \beta}^{*}(-\vec{l})=G_{\alpha \beta}(\vec{l})
$$


Il est possible de transformer la formule (41.4) de manière à réduire le domaine d'intégration ia la nappe positive $C^{+}$du cône isotrope. Il vient ainsi:

$$
F_{\alpha \beta}\left(\overrightarrow{x)}=\frac{1}{(2 \pi)^{3}} \int_{C^{+}}\left(G_{\alpha \beta} \overrightarrow{(l)} e^{\vec{\imath} \cdot \vec{x}}+G_{\alpha \beta}(-\vec{l}) e^{-i \vec{l} \cdot \vec{x})} d \mathrm{Q}(\vec{l})\right.\right.
$$

soit, d'après $(42-1)$,

$$
F_{\alpha \beta}(\vec{x})=\frac{1}{(2 \pi)^{3}} \int_{C^{+}}\left(G_{\alpha \beta}(\vec{l}) e^{i \vec{l} \cdot \vec{x}}+G_{\alpha \beta}^{*}\left(\vec{l} \vec{l} e^{-\vec{l} \cdot \vec{x}}\right) d Q(\vec{l}) .\right.
$$

Sur la formule $(42-2)$, le caractère hermitian des valeurs de $F$ se tronve en évidence. L'hypothèse $b$ se traduit par les relations:

$$
S l_{\alpha} G_{\beta Y}=0 \quad l^{\alpha} G_{\alpha \beta}=0 .
$$

Supposons que $G_{\alpha \beta}$ soit une 2-forme à valeurs scalaires satisfaisant aे (41-3). Ces relations expriment que la z-forme envisagée est singulière. Désignous par $\left.\overrightarrow{u^{(1)}}, \vec{l}\right)$ et $\overrightarrow{n^{(2)}(\vec{l})}$ denx vecteurs orthogonaux normés arbitraires du 3-plan tangent au còne isotrope le long de $\vec{l}$. Ces vecteurs définissent un 2-plan orienté dans l'espace et le 2-plan orthogonal orienté dans le temps contient $\vec{l}$. Si $\overrightarrow{e_{0}}$ est un vecteur unitaire arbitrairc de ce 2 -plan, on obtient un repère orthonormé $\left(\vec{e}_{\alpha}\right)$ tel que:

$$
\frac{\vec{l}}{l^{0}}=\overrightarrow{e_{0}}+\overrightarrow{e_{1}} \quad \overrightarrow{e_{2}}=\overrightarrow{n^{(1)}} \quad \overrightarrow{e_{3}}=\overrightarrow{n^{(2)}}
$$

De l'étude du $\S 7$ (voir en particulier (7.3), il résulte que pour que $G_{\alpha \beta}$ satisfasse $(42-3)$, il fant et il suffit que:

$$
\left.G_{\alpha \beta} \vec{l}\right)=\sum_{i} \alpha(i, \vec{l})\left(l_{\alpha} n_{\beta}^{(i)}-l_{\beta} n_{\alpha}^{(i)}\right)
$$

où les indices $i, j, \ldots$ prennent les valeurs 1 et 2 et où les $a(i, \vec{l})$ sont des scalaires.

Par l'introduction $d$ 'une forme linéaire sur $M$ a valeurs scalaires on voit immédiatement que si $G_{\alpha \beta}$ est une 2 -forme à valeurs dans $M$ satisfaisant $(42-3)$, la formule $(42.5)$ est encore valable $\dot{y}$ condition de prendre pour les $\mathbf{a}(i, \vec{l})$ des élements de $M$.

La quantification du champ $F$ s'effectuer: en postulant les conditions de crochet:

$$
\left\{\begin{array}{l}
{[a(i, \vec{l}), a(j, \vec{l})]=0} \\
{\left[a^{*}(i, \vec{l}), a\left(j, l^{\prime}\right)\right]=\frac{\vec{h}}{i} \delta_{i j} \delta_{0} \vec{l}, \overrightarrow{\left.l^{\prime}\right)}}
\end{array}\right.
$$


A. Lichnerowicz: Ondes et radiations électromagnétiques, eto.

où $\vec{l}$ et $\vec{l}$ sont deux vecteurs de $C^{+}, \delta_{i j}$ le symbole de Kronecker et $\delta_{0}$ la mesure de Dirac relative an cône isotrope muni de l'élément de volume $d Q$ On peut obtenir un formalisme plus condensé en introduisant des indices $A, B$ prenant les valeurs +1 et -1 et en posant:

$$
\left.a^{A}(i, \vec{l})=a(i, \vec{l}) \text { pour } A=1 \quad a^{A(i,} \vec{l}\right)=a^{*}(i, \vec{l}) \text { pour } A=-1 .
$$

Avec ces notations les conditions de quantification $(42-6)$ s'écrivent:

$$
\left[a^{-A}(i \vec{l}), a^{B}\left(j, \overrightarrow{l^{\prime}}\right)\right]=\frac{\bar{h}}{i} A \delta_{A B} \delta_{i j} \delta_{\Omega}(\vec{l}, \vec{l})
$$

\section{Une formule auxiliaire.}

Dans le calcul des relations de commutation auxquelles satisfait, en vertu de (42-7) le champ $F$, nous serons amenés à évaluer le tenseur défini à partir de $\vec{l}, \overrightarrow{n^{(1)}(\vec{l})}$ et $\overrightarrow{n^{(2)}(\vec{l})}$ par:

$$
P_{\alpha \beta, \lambda \mu} \vec{l}(\vec{l})=\Sigma \delta_{i j}\left(l_{\alpha} n_{\beta}^{(i)}-l_{\beta} n_{\alpha}^{(i)}\right)\left(l_{2} n_{\mu}^{(j)}-l_{\mu} n\left({ }_{\lambda}^{(j)}\right)\right.
$$

Pour cette évaluation, partons du repère orthonormé introduit an $\$ 42$ et satisfaisant à (42.4); posons

$$
\overrightarrow{e_{0}}=\vec{u} \quad \overrightarrow{e_{1}}=\frac{l}{l^{0}}-\vec{u}=k \vec{l}-\vec{u} \quad\left(k=1 / l^{0}\right) .
$$

A partir des composantes des vecteurs de ce repère orthonormé, le tenseur métrique $\eta_{\alpha \beta}$ de $V_{4}$ peut s'exprimer par:

$$
\eta_{\alpha \beta}=u_{\alpha} u_{\beta}-\left(k l_{\alpha}-u_{\alpha}\right)\left(k l_{\beta}-u_{\beta}\right)-n_{\alpha}^{(1)} n_{\beta}^{(1)}-n_{\alpha}^{(2)} n_{\beta}^{(2)}
$$

On en déduit:

$$
\sum_{i, j} \delta_{i j} n_{\alpha}^{(i)} n_{\beta}^{(i)}=n_{\alpha}^{(1)} n_{\beta}^{(1)}+n_{\alpha}^{(2)} n_{\beta}^{(\beta)}=l_{c}\left(l_{\alpha} u_{\beta}+l_{\beta} u_{\alpha}\right)-k^{2} l_{\alpha} l_{\beta}-\eta_{\alpha \beta} .
$$

En développant le second membre de $(43-1)$, on obtient:

$$
\begin{gathered}
P_{\alpha \beta, \lambda_{\mu} \mu}(\vec{l})=l_{\alpha} l_{\lambda}\left\{k\left(l_{\beta} u_{\mu}+l_{\mu} u_{\beta}\right)-k^{2} l_{\beta} l_{\mu}-\eta_{\beta_{\beta}}\right\}+l_{\beta} l_{\mu}\left\{k\left(l_{\alpha} u_{\lambda}+l_{\lambda} u_{\alpha}\right)-k^{2} l_{\alpha} l_{\lambda}-\eta_{\alpha \lambda}\right\} \\
-l_{\alpha} l_{\mu}\left\{k\left(l_{\beta} u_{\lambda}+l_{\lambda} u_{\beta}\right)-k^{2} l_{\beta} l_{\lambda}-\eta_{\alpha \beta}-l_{\beta} l_{\lambda}\left\{k\left(l_{\alpha} u_{\mu}+l_{\mu} u_{\alpha}\right)-k^{2} l_{\alpha} l_{\mu}-\eta_{\alpha_{\mu}}\right\}\right.
\end{gathered}
$$

Après simplification, il vient ainsi la formule:

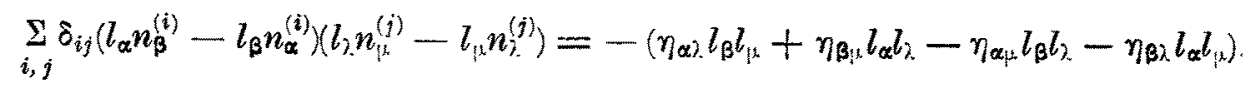




\section{Les relations de commutation.}

Au point $\vec{x}$ de $V_{4}, F_{\alpha \beta}(\vec{x})$ peut s'écrire, en vertu de $(42-5)$

$$
F_{\alpha \beta} \overrightarrow{(x)}=\frac{1}{(2 \pi)^{3}} \underset{A, i}{\mathrm{~V}} \int_{C^{+}} \alpha^{A}(i, \vec{l}) e^{i A \vec{\imath} \cdot \vec{x}}\left(l_{\alpha} n_{\beta}^{(i)}-l_{\beta} n_{\alpha}^{(i)}\right) d \Omega \vec{l} \overrightarrow{.}
$$

Soit $\overrightarrow{x^{\prime}}$ un autre point de $V_{4}$ pour lequel:

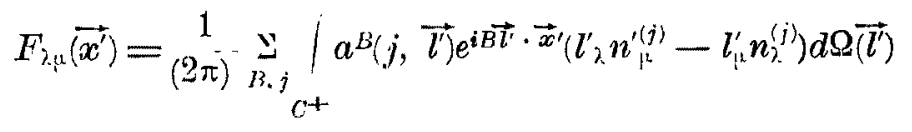

où les $\overrightarrow{n^{(j}}(\vec{l})$ sont des vecteurs orthogonaux normés tangents à $C$ le long de $\vec{l}$. Evaluons le crochet

$$
\begin{aligned}
& {\left[F_{\alpha \beta}\left(\overrightarrow{x)}, F_{\lambda, k}\left(\vec{x}^{\prime}\right)\right]=\frac{1}{(2 \pi)^{\beta}} \iint_{C^{+} C^{+}}\left[a^{-A}(i, \vec{l}), a^{B}(j, \vec{l})\right] e^{i\left(-\overrightarrow{A l} \cdot \vec{x}+\overrightarrow{B l^{\prime}} \vec{x}^{\prime}\right)}\right.}
\end{aligned}
$$

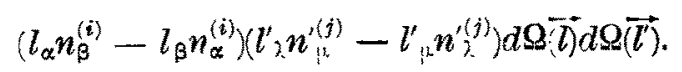

$D^{\circ}$ après $(42-7)$, il vient:

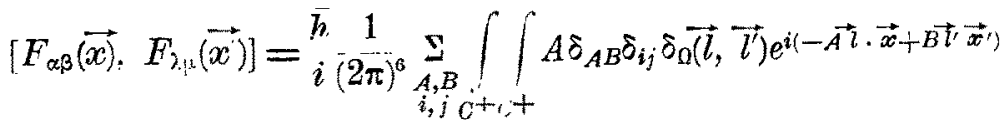

$$
\begin{aligned}
& \left(l_{\alpha} n_{\beta}^{(i)}-l_{\beta} n_{\alpha}^{(i)}\right)\left(l_{,}^{\prime} n_{i^{\prime}}^{\prime(j)}-l_{\mu}^{\prime} n_{\lambda}^{\prime(i)}\right) d \Omega(\vec{l}) d \mathbf{Q}\left(\overrightarrow{l^{\prime}}\right) .
\end{aligned}
$$

En intégrant par rapport à la variable $\overrightarrow{l^{\prime}}$, on obtient:

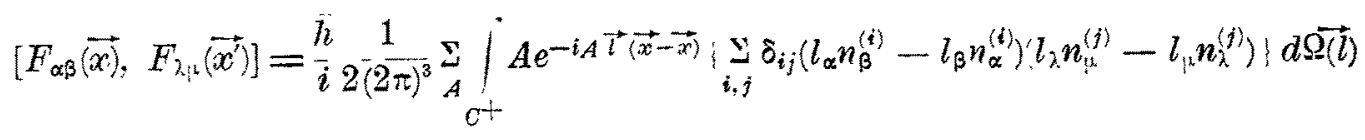

De la formule préliminaire (43-2) on déduit ainsi:

$$
\begin{gathered}
{\left[F_{\alpha \beta}(\vec{x}), F_{\lambda \beta}\left(\overrightarrow{x^{\prime}}\right)\right]=-\frac{\breve{h}}{i} \frac{1}{2(2 \pi)^{3}} \underset{A}{\mathrm{Q}} \int_{C^{+}} A e^{-i A \vec{l} \cdot\left(\vec{x}-\vec{x}^{\prime}\right)}} \\
\left(\eta_{\alpha \beta} l_{\beta} l_{\mu}+\eta_{\beta \mu} l_{\alpha} l_{\lambda}-\eta_{\alpha \mu} l_{\beta} l_{\lambda}-\eta_{\alpha \lambda} l_{\alpha} l_{\mu}\right) d \Omega(\vec{l}) .
\end{gathered}
$$

Nous sommes ainsi conduits à introduire la distribution invariante définie par:

$$
D(\vec{x})=\frac{1}{2(2 \pi)^{3}} \sum_{C^{+}} A e^{-i A \vec{l} \cdot \vec{x}} d \Omega(\vec{l})
$$


qui n'est autre que le "propagateur» de JoRdan-PaUli. On obtient immé. diatement:

$$
\partial_{\alpha} D \overrightarrow{(x)}=-\frac{i}{2(2 \pi)_{A}^{3}} \Sigma \int_{C^{+}} e^{-i \overrightarrow{A l} \cdot \vec{x}} l_{\alpha} d \Omega(\vec{l})
$$

et

$$
\partial_{\alpha} \partial_{\beta} D \overrightarrow{(\vec{c})}=-\frac{1}{2(2 \pi)^{3}} \Sigma \int_{C^{+}} A e^{-i A \vec{l} \cdot \vec{x}} l_{\alpha} l_{\beta} d Q(\vec{l}) .
$$

Par suite la formule (44-2) peut s'écrire:

$$
\left[F_{\alpha \beta}(\vec{x}), F_{\lambda \mu}\left(\overrightarrow{x^{\prime}}\right)\right]=\frac{\bar{h}}{i}\left(\eta_{\alpha \lambda} \partial_{\alpha} \partial_{\mu}+\eta_{\beta \mu} \partial_{\alpha} \partial_{\lambda}-\eta_{\alpha, \lambda} \partial_{\beta} \partial_{\lambda}-\eta_{\beta \lambda} \partial_{\alpha} \partial_{\mu}\right) D(\vec{x}-\vec{x})
$$

où les dérivations ont lieu par rapport à la variable $\vec{x}$. La formule (44-4) pent encore s'écrire en substituant à $\eta_{\alpha \lambda}$ le produit scalaire $\overrightarrow{e_{\alpha}} \cdot \overrightarrow{e_{\lambda}}$ des vecteurs du repère et en introduisant des dérivations par rapport aux coordonnées $d u$ point $\overrightarrow{x^{\prime}}$.

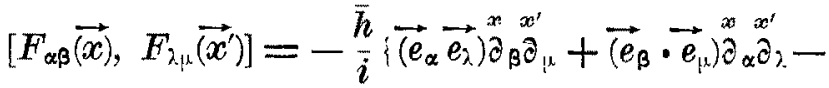

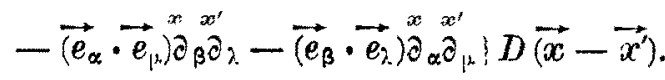

Dans (44-4) ou (44-5) nous retrouvons la relation de commutation classique de la théorie quantique du champ électromagnetique $\left({ }^{13}\right)$. Comme on le vórifie immédiatement, le crochet obtenu est bien compatible avec les équations (41-2): si $K_{\alpha \beta, \lambda_{k}}$ désigne le crochet

$$
S \partial_{\gamma} K_{\alpha \beta, \lambda_{\beta}}=0 \quad \partial_{\alpha} K_{\beta, \lambda_{\mu}}=0
$$

où $S$ indique une sommation après permutation circulaire sur le trois indices $\alpha, \beta, \gamma$. Cette quantification définit une représentation nnitaire irréductible du groupe de LoRENiz inhomogène qui peut être caractérisée par sa restriction au "petit groupe" au sens de WIGNer (sous groupe laissant invariant un vecteur isotrope). Comme on sait la représentation obtenue est caractérisée par une masse nulle et un spin égal à 1 .

\section{Forme de la relation de commutation en repères arbitraires.}

Supposons les voisinages des points $\vec{x}$ et $x^{\prime}$ rapportés à denx repères différents $\left(\vec{e}_{\alpha}\right)$ et $\left(\vec{e}_{\lambda^{\prime}}\right)$ arbitraires. Nous rapportons ainsi le champ électromagnétique en $\vec{x}$ au repère $\overrightarrow{\left(e_{\alpha}\right)}$ et le champ électomagnétique en $\overrightarrow{x^{\prime}}$ au repère

(11) Voir par exemple G. Wentzer, Quantum theory of fields, p. 115. 
$\left(\overrightarrow{e_{\lambda}}\right)$. Par multiplication par les matrices de changements de repères, la formule (44-5) peut sećrire en supprimant les indices $x$ et $x^{\prime}$ devenus inutiles.

$$
\begin{aligned}
& {\left[F_{\alpha \beta}(\vec{x}), F_{\lambda^{\prime \mu^{\prime}}}\left(\overrightarrow{x^{\prime}}\right)\right]=-\frac{\bar{h}}{i}\left\{\left(\overrightarrow{e_{\alpha}} \cdot \overrightarrow{\left.e_{\lambda^{\prime}}\right)} \partial_{\beta} \partial_{\mu^{\prime}}+\overrightarrow{\left(e_{\beta}\right.} \cdot \overrightarrow{\left.e_{\alpha^{\prime}}\right)} \partial_{\alpha} \partial_{\lambda^{\prime}}-\right.\right.} \\
& \left.\left.-\overrightarrow{\left(e_{\alpha}\right.} \cdot \overrightarrow{e_{1 \mu^{\prime}}}\right) \partial_{\beta} \partial_{\lambda^{\prime}}-\overrightarrow{\left(e_{\beta}\right.} \cdot \vec{e}_{\lambda^{\prime}}\right) \partial_{\alpha^{\prime}} \partial_{\mu^{\prime}} \mid \overrightarrow{\left(\vec{x}-\overrightarrow{x^{\prime}}\right)}
\end{aligned}
$$

où les deux membres représentent un bitenseur, tenseur antisymétrique d'ordre 2 en $\vec{x}$ et tenseur antisymétrique d'ordre 2 en $\overrightarrow{x^{\prime}}$. Le second membre met en evidence le bitenseur défini par les produits $\left(\overrightarrow{e_{\alpha}} \cdot \overrightarrow{e_{\lambda^{\prime}}}\right)$ et dont il est aisé d'ob. tenir une expression intéressante; on a en effet:

$$
\overrightarrow{x^{\prime}}-\vec{x}=x^{\lambda^{\prime}} \overrightarrow{e_{\lambda^{\prime}}}-x^{\alpha} \overrightarrow{e_{\alpha}} \text {. }
$$

Par suite si $s$ désigne l'intervalle spatio-temporel joignant le point $\vec{x}$ an point $\overrightarrow{x^{\prime}}$.

$$
\left.s^{2}=\overrightarrow{\left(x^{\prime}\right.}-\vec{x}\right)^{2}=x^{\prime \prime} x^{\mu^{\prime} \vec{e}_{\lambda^{\prime}}} \overrightarrow{e_{\mu^{\prime}}}+x^{\alpha} x^{\beta} \overrightarrow{e_{\alpha}} \cdot \overrightarrow{e_{\beta}}-2 x^{\alpha} x^{\lambda^{\prime}} \overrightarrow{e_{\alpha}} \cdot \overrightarrow{e_{\lambda^{\prime}}}
$$

Par dérivation il vient:

$$
\hat{c}_{\alpha} \partial_{\lambda^{\prime}} s^{2}=-\overrightarrow{2 e_{\alpha}} \cdot \overrightarrow{e_{\lambda^{\prime}}} .
$$

Ainsi si nous introduisons le bitenseur en $\vec{x}$ et $\vec{x} c^{\prime}$ défini par:

$$
\theta_{\alpha\rangle^{\prime}}= \pm \frac{1}{2} \partial_{\alpha} \hat{c}_{\lambda^{\prime}} s^{2}
$$

on obtient pour la relation de commutation la forme:

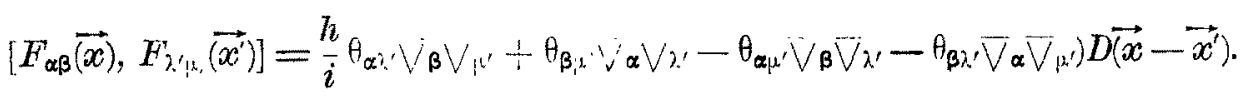

Sous cette forme la relation est valable en repères mobiles quelconques et en particulier si l'on rapporte l'espace-temps a des cordonnées curvilignes locales $\overrightarrow{\left(x^{\alpha}\right)}$ au voisinage de $\vec{x}$ et $\overrightarrow{\left(x^{\prime}\right)}$ au voisinage de $\overrightarrow{x^{\prime}}$.

\section{B. LE CHAMP GRAVITATIONNEL.}

\section{I.es équations de champ.}

Considérons un espace-temps riemamien $V_{4}$ et désignons par $R_{\beta, \lambda_{k}}$ sun tenseur de conrbure. Celui-ci jouit des propriétes de symétrie suivantes:

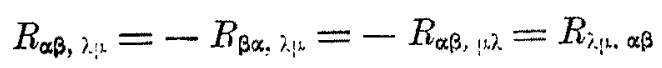

et satisfait à l'identité :

$$
S R^{\lambda}{ }_{\alpha, \beta_{\gamma}}=0
$$


qui n'est autre que la condition d'intégrabilitê relative à la torsion dans le cas où celle-ci est nulle. Nous supposons que l'espace-temps envisagé $V_{4}$ satisfait aux équations d' EINSTEIN

$$
R_{\alpha \beta}=0 .
$$

L'analogie avec les équations de Maxwell et l'étude fait de l'état de radiation pure nous conduisent à adopter comme équations de champ.:

$$
\text { (a) } S \nabla_{\alpha} R^{\lambda}{ }_{\mu, \beta \gamma}=0 ; \quad \text { (b) } \bar{V}_{\alpha} R_{\beta, \lambda \mu}^{\alpha}=0
$$

où $\bar{V}_{\alpha}$ est l'opérateur de dérivation covariante dans la connexion riemannienne; (46-4) $)_{a}$ n'est autre que l'identité de BranchI, condition d'intégrabilité des équations reliant le tenseur de courbure à la connexion. De $(46-4)_{a-}$, on déduit par contraction:

$$
\bar{V}_{\alpha} R_{\mu, \beta \gamma}^{\alpha}=\bar{V}_{\beta} R_{\mu \gamma}-\bar{V}_{r} R_{\mu \beta}
$$

et l'on voit que (46-3) entraìne $(46 \cdot 4)_{b}$

$\left.2^{\circ}\right)$ Nous nous proposons de montrer qu'inversement si les équations de champ (46-4) sont satisfaites, (46-3) peut ètre considérée comme une simple condition initiale. D' une manière plus précise, soit $\Sigma$ une hypersurface de $V_{4}$ orientée dans l'espace et sur laquelle $R_{\alpha \beta}$ s'annule identiquemet; si les équations (46-4) sont satisfaites, nous allons établir que $R_{\alpha \beta}$ est aussi nécessairement nul en dehors de $\Sigma$.

Soit $x^{0}=0$ l'équation locale de $\Sigma$; cette hypersurface étant orientée dans l'espace, on a $g^{00}>0$. De $(46 \cdot 4)_{b}$ il résulte d'après $(46.5)$

$$
\bar{V}_{\beta} R_{\mu \varphi}-\nabla_{Y} R_{\mu \beta}=0
$$

et en contractant $(46.4)_{b}$, il vient:

$$
\bar{\nabla}_{\alpha} R^{\alpha_{\lambda}}=0
$$

qui peut encore s'écrire:

$$
g^{\alpha \beta} \bar{\vee}_{\alpha} R_{\beta \lambda}=0
$$

Désignons par $u, v, \ldots$ des indices prenant les valeur 1, 2, 3. En explicitant le premier membre de (46.7) on a:

$$
g^{00} \bar{\vee}_{0} R_{0 \lambda}+g^{\prime u}\left(\nabla_{0} R_{u \lambda}+\nabla_{u} R_{0 \lambda}\right)+g^{u v} \bar{V}_{u} R_{v \lambda}=0
$$

soit d'après $(46-6)$ :

$$
g^{00} \bar{V}_{0} R_{0 \lambda}=-2 g^{0 x} \nabla_{u} R_{0 \lambda}-g^{3 v} \bar{\vee}_{u} R_{v \lambda} .
$$


En faisant dans (46-6) $\beta=0, \gamma=u$ et dans $(46.8) \lambda=0$, on obtient le système linéaire et homogène aux dérivées partielles du premier ordre:

$$
\left\{\begin{array}{l}
\bar{\nabla}_{0} R_{\mu u}=\bar{\nabla}_{u} R_{\mu \nu} \\
g^{00} \bar{\vee}_{0} R_{00}=-2 g^{0 u} \bar{V}_{u} R_{00}-g^{u v} \bar{\nabla}_{u} R_{r 0}
\end{array}\right.
$$

où $g^{00}$ est $\neq 0$. Pour une donnéc initiale $R_{\alpha \beta}$ nulle sur $\Sigma$, ce système n'admet d'autre solution que la solution nulle, ce qui démontre la propriété.

$\left.3^{\circ}\right)$ Supposons $V_{4}$ portée par un espace-temps minkowskien. Si le champ de gravitation envisagé est faible, le tenseur métrique $g_{\alpha \beta}$ de $V_{4}$ peut s'écrire:

$$
g_{\alpha \beta}=\eta_{\alpha \beta}+\varepsilon \psi_{\alpha \beta}
$$

où $\eta_{\alpha \beta}$ définit la métrique minkowskienne et où $\varepsilon$ est l'infiniment petit principal. Si $V_{4}$ est rapporté à un repère orthonormé relativement à la mé. trique $\eta_{\alpha \beta}$, les coefficients correspondants de la connexion riemannienne $V_{4}$ sont de l'ordre de $\varepsilon$, ainsi que les composantes du tenseur de courbure. Les indices étant élevés et abaissés à l'aide $d u$ tensenr $\eta_{\alpha \beta}$, la partie principale $H_{\beta, \lambda, \mu}^{\alpha}$ du tenseur de courbure satisfait aux identités (46-1) et (46.2) et est astreint aux équations déduites de (46.4) en substituant aux dérivées covariantes relatives à $g_{\alpha \beta}$ les dérivées ordinaires.

\section{Conditions de quantification.}

Prenons donc pour espace-temps $V_{4}$ l'espace-temps de Minkowski que nous supposons toujours rapporté à des repères orthonormés $\left.\vec{e}_{\alpha}\right)$. Nous som. mes conduits à décrire le champ de gravitation au moyen d' un tenseur $H_{\alpha \beta,}$, satisfaisant aux identités

$$
H_{\alpha \beta, \lambda_{i k}}=-H_{\beta \alpha, \lambda_{\mu}}=-H_{\alpha \beta, \mu \lambda}=H_{\lambda \mu, \alpha \beta}
$$

et à l'identité:

$$
S H^{\lambda_{\alpha, \beta y}}=0
$$

les indices étant élevés et abaissés à l'aide de la métrique $\eta_{\alpha \beta}$ de l'espace-temps. Nous prendons comme équations de champ:

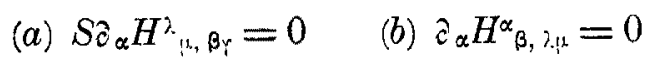

et ajouterons à ces équations la condition supplémentaire:

$$
H_{\alpha \beta}=H_{\alpha, \beta \beta}=0 \text {. }
$$

Le raisonnement mème du $2^{\circ}$ paragraphe precedent, où l'on substitute $R_{\alpha \beta}, \gamma_{k}$ le tenseur $H_{\alpha \beta}, \lambda_{\mu}$, montre qu'il suffit, pour une solution de (47-3), de vérifier cette condition sur une hypersurface $\Sigma$ orientée dans l'espace.

Un tenseur ordinaire tel que $H$ peat être identifié à une forme multili. néaire $H_{\alpha \beta, \lambda, \mu} V_{(1)}^{\alpha} V_{(2)}^{\beta} V_{(3)}^{\lambda} \Gamma_{(4)}^{\beta \mu}$ à valeurs scalaires. Substituons-lui une forme 
A. LICINERowiCz: Ondes et radiations électromagnétiques, etc.

multilinéaire désignée par la mềne notation, mais à valeurs dans l'espace vectoriel $M$ et satisfaisant bien entendu aux identités $(47-1)$ et (47-2). De manière analogue au cas électromagnélique, nous supposerons que:

a) les valeurs de $H$ sont des opérateurs hermitiens

b) $H$ satisfaisant aux equations (47-3).

Sous des hypothèses simples permettant l'introduction de la transformation de Fourier, et avec des notations analogues a celles utilisées dans le cas électromagnétique, $H$ peut être mis sous la forme:

$$
H_{\alpha \beta, \lambda \mu} \overrightarrow{(x)}=\frac{1}{(2 \pi)^{3}} \int_{C+}\left(K_{\alpha \beta, \lambda, \mu} \vec{l} \vec{l}\right) e^{i \vec{l} \cdot \vec{x}}+K_{\alpha, \beta, \lambda \mu}^{*} \overrightarrow{(l)} e^{-\overrightarrow{i l} \cdot \vec{x})} d \overrightarrow{Q(\vec{l})}
$$

où $\vec{x} \in V_{4}$ ef ou $K_{\alpha \beta, \lambda \mu}$ est un tenseur à valeurs dans $M$. L'hypothèse $b$ se traduit par les relations

$$
\text { (a) } S l_{\alpha} K_{\beta \gamma, \lambda \mu}=0 \quad \text { (b) } l^{\alpha} K_{\alpha \beta, \lambda \mu}=0 .
$$

Supposons que $H_{\alpha \beta, \lambda}$ soit an tenseur ordinaire jouissant des propriétés de symétrie ${ }_{3 \rightarrow}(47-1)$ et satisfaisant à $(47.3)$; lo tenseur $K_{\alpha \beta,} \lambda_{\mu}(\vec{l})$ correspondant jouit des mèmes propriétés de symétrie et d'après (21.9) les solutions de (47-6) sont données par:

$$
K_{\alpha \beta, \lambda, \mu}(\vec{l})=\sum_{i, j} \alpha(i, j, \vec{l})\left(l_{\alpha} n_{\beta}^{(i)}-l_{\beta} n_{\alpha}^{(i)}\right)\left(l_{\lambda} n_{\mu}^{(j)}-l_{\beta} n_{\lambda}^{(j)}\right)
$$

avec

$$
a(i, j, \overrightarrow{l)}=\alpha(j, i, \vec{l})
$$

par raison de symétrie.

Par l'introduction d' une forme linéaire sur $M$ à valeurs scalaires, on voit encore que si $K_{\alpha \beta, \lambda \mu}$ est un tenseur à valeurs dans $M$ jouissant des propriétés de symétrie précisées et satisfaisant à (47.6), la formule (47.7) est toujours valable, à condition de prendre pour les $a(i, j, \vec{l})$ des éléments de $M$.

La quantification du champ $H$ s'effectuera en postalant les conditions de crochet:

$$
\left\{\begin{array}{l}
\left\lfloor a(i, j, \vec{l}), a\left(h, k, \vec{l}^{\prime}\right)\right]=0 \\
\left.\left[a^{*}(i, j, \vec{l}), a^{\prime} h, k, \vec{l}^{\prime}\right)\right]=\frac{\bar{h}}{i}\left(\delta_{i h} \hat{\partial}_{j k}+\delta_{i k} \hat{o}_{j h}-\delta_{i j} \delta_{h k}\right) \tilde{o}_{0}\left(\vec{l}, \overrightarrow{\left.l^{\prime}\right)}\right.
\end{array}\right.
$$

où $\vec{l}$ et $\vec{l}$ sont des vecteurs de $C^{+}$. Au moyen du formalisme condensé intro. duit dans le cas électromagnétique, on peut traduire (47.8) par:

$$
\left[a^{-A}(i, j, \vec{l}), a^{B}(h, k, \vec{l})\right]=\frac{h}{i} A_{A B}\left(\hat{\delta}_{i h} \delta_{j k}+\delta_{i k} \delta_{j h}-\delta_{i j} \delta_{h k}\right) \delta_{\Omega}(\vec{l}, \vec{l}) .
$$




\section{Les relations de commutations.}

Au point $\vec{x}$ de $V_{4}, H_{\alpha \beta, ~ \gamma o \delta}(\overrightarrow{x)}$ peut s'écrire, en vertu de $(47 \cdot 5)$ et $(47.7)$.

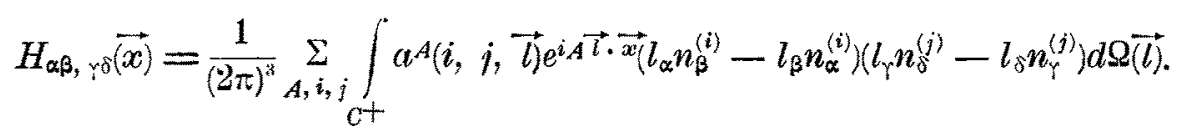

Soit $\overrightarrow{x^{\prime}}$ un autre point de $V_{4}$ pour lequel:

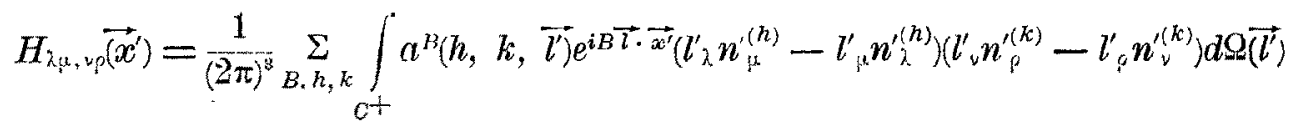

oú les $\overrightarrow{n^{\prime}(h)}\left(\overrightarrow{\left.l^{\prime}\right)}\right.$ sont des vecteurs orthogonaux tangents at $C$ le long de $\overrightarrow{l^{\prime \prime}}$. D'après $(47-9)$, on a:

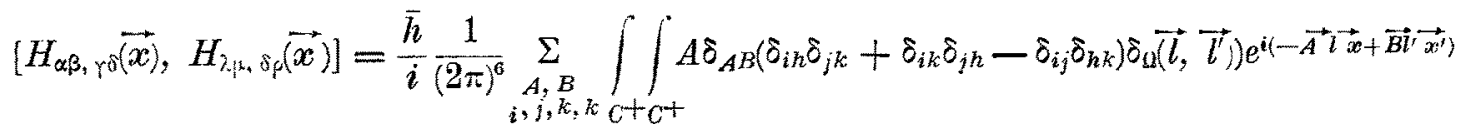

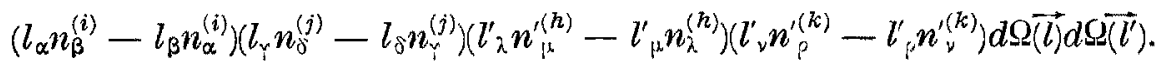

En intégrant par rapport à la variable $\vec{l}$, on obtient:

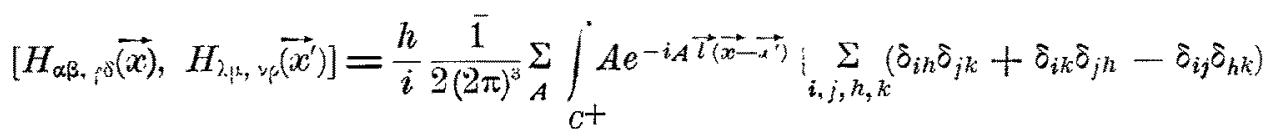

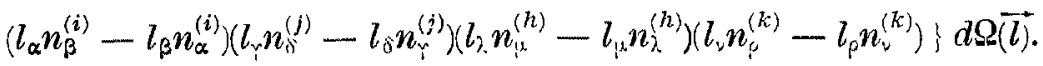

De la formule auxiliaire (43-2), il résulte ainsi:

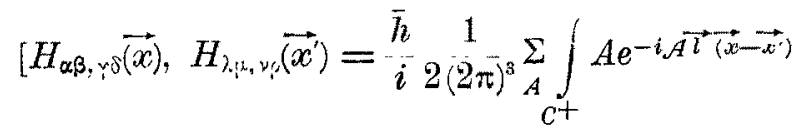

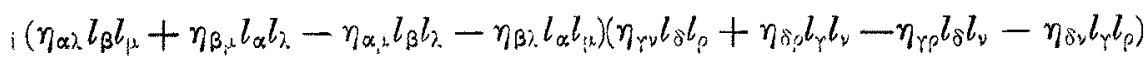

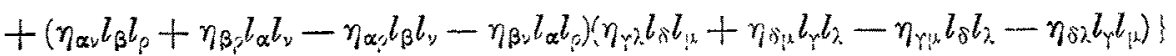

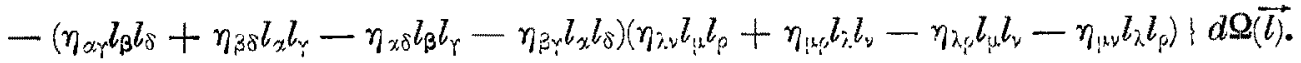

De l'introduction du propagateur $\overline{D(x)}$ de JoRDAN-PAULI, on déduit:

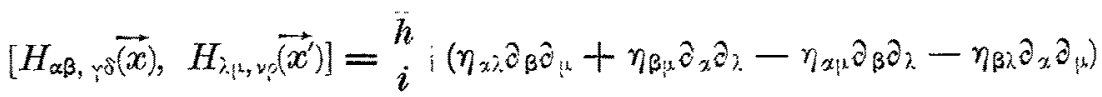

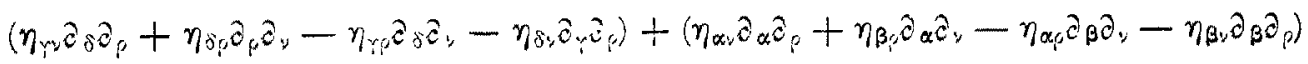

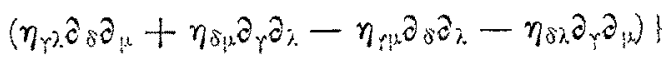

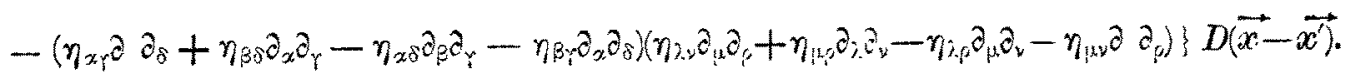


Nous avons ainsi obtenu la relation de commutation relative au champ $H$. On vérifie aisément que si $H$ satisfait à l'identité (47-2), la relation obtenne est compatible avec cette identité. Elle est bien entendu compatible avec les equations de champ (47-3). L'étude, selon WIGNER, da "petit groupe» montre aisément que la quantification précédente definie par (47-9) donne en général un mélange de particules de masses nulles et de spin 0 et 2 . Si l'on introduit la condition supplémentaire $H_{\alpha \beta}=0$, on a nécessairement $K_{\alpha \beta}=K_{\alpha_{\alpha, \beta}}=0$ et on déduit de (47.7)

$$
\sum_{i, j} \delta_{i j} a(i, j, \vec{l})=0
$$

Soit la condition de trace:

$$
a(1,1, \vec{l}+(2,2, \vec{l})=0
$$

qui ne laisse subsister que la représentation caractérisée par une masse nulle et un spin égal ì̀ 2 .

\section{Interprétation du tenseur $H$ en termes de métrique.}

10) Dans l'espace-temps minkowskien rapporté ì un repère orthonormé relativement à $\eta_{\alpha \beta}$. considérons le champ de tenseurs ordinaire $H^{\lambda_{\mu}, \beta \alpha}$ satisfaisant aux identités algébriques (47.1) et (47.2) et aux équations de champ (47-3), ainsi qu'à la condition supplémentaire $H_{\alpha \beta}=0$. D'après $(47-3)_{\mathfrak{a}}$, il existe un système de quantités $\Gamma_{\alpha \beta}^{\lambda}$ telles que:

$$
H_{\mu \mu, \alpha \beta}^{\lambda}=\partial_{\alpha} \Gamma_{i, \beta}^{\hat{\lambda}}-\partial_{\beta} \Gamma_{\mu \alpha}^{\lambda}
$$

ce système de quantités se trouvant défini à la transformation près:

$$
\Gamma_{\alpha \beta}^{\lambda} \rightarrow \Gamma_{\alpha \beta}^{\lambda}+\partial_{\beta} A_{\alpha}^{\lambda}
$$

ox̀ les $A_{\alpha}^{\lambda}$ sont arbitraires. Posons

$$
S_{\alpha \beta}^{\lambda}=\frac{1}{2}\left(\Gamma_{\alpha \beta}^{\lambda}-\Gamma_{\beta \alpha}^{\lambda}\right)
$$

De (49-1) écrite sous la forme:

$$
H_{\alpha, \beta_{\gamma}}^{\lambda_{\gamma}}=\partial_{\beta} \Gamma_{\alpha_{Y}}^{\lambda}-\partial_{Y} \Gamma_{\alpha \beta}^{\grave{\lambda}}
$$

on tire par sommation, après permutation circulaire sur $\alpha, \beta, \gamma$

$$
S \partial_{\gamma}^{\prime} S_{\alpha \beta}^{\lambda}=-\frac{1}{2} S H_{\alpha, \beta \gamma}^{\prime}=0
$$


d'après l'identité (47-2). Il en résulte qu'il existe un système de quantités $B_{\alpha}^{\lambda}$ telles que:

$$
2 S_{\alpha \beta}^{\lambda}=\hat{c}_{\alpha} B_{\beta}^{\lambda}-\partial_{\beta} B_{\alpha}^{\lambda}
$$

ce système de quantités étant défini ì la transformation près:

$$
B_{\alpha}^{\lambda} \rightarrow B_{\alpha}^{\lambda}+\partial_{\alpha} \rho^{\lambda} \text {. }
$$

De $(49-3)$; il rësulte:

$$
\Gamma_{\alpha \beta}^{\lambda}+\partial_{\beta} B_{\alpha}^{\lambda}=\Gamma_{\beta \alpha}^{\lambda}+\partial_{\alpha} B_{\beta}^{\lambda}
$$

En substituant aux $\Gamma_{\alpha \beta}^{\lambda}$ initiaux les premiers membres de (49-5), ce qui est permis par la transformation (49-2). on voit que les $\Gamma_{\alpha \beta}^{\lambda}$ satisfaisant a (49-1) peuvent ètre astreints à ètre symétriques par rapport a lears deux indices inférieurs. Ils se trouvent alors définis à la transformation près:

$$
\Gamma_{\alpha \beta}^{\lambda}-\Gamma_{\alpha \beta}^{\grave{\lambda}}+\hat{\mathfrak{z}}_{\beta} \hat{\partial}_{\alpha} \hat{p}^{\gamma} \text {. }
$$

Elevons et abaissons les indices au moyen du tenseur $\eta_{i_{i}}$ et posons en particulier:

$$
\Gamma_{\alpha, \beta \beta}=\eta_{j, k \beta} \Gamma_{\alpha \beta \beta}^{\dot{\gamma}}
$$

La relation (49-1) peut se mettre sons la forme:

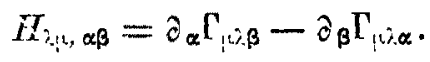

De l'identité (47-1) rérifiée par $H$, il résulte:

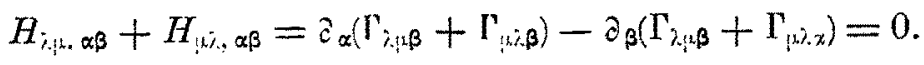

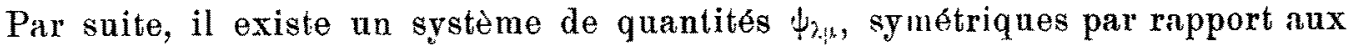
indices $\lambda$ ef $\mu$, telles que:

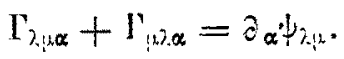

Compte-tenu de $(49.6), \psi_{, \mu}$ est défini à la transformation près:

$$
\psi_{\lambda \mu} \rightarrow \psi_{\lambda i^{\alpha}}+\mathfrak{z}_{\lambda \varphi_{\mu}}+\partial_{\mu} \varphi_{\lambda}+\text { const. }
$$

Des propriétés de symétrie des $\Gamma$ et de (49-7), on déduit:

$$
\Gamma_{i, \mid \mu,}=[\lambda \mu, v]
$$

où le second membre désigne l'algorithme de CHRIsToFres appliqué au système des $\psi_{\lambda, \mu}$. Ainsi, d'après les hypothèses faites sur $H$, il existe un 
système de quantités $\psi_{\lambda_{j k}}$ (avec $\psi_{\lambda \mu \alpha}=\psi_{\mid \lambda \lambda}$ ), définies à la transformation (49-8) près, telles que les quantités (49-9) qui s'en déduisent vérifient:

$$
H_{\lambda \mu \alpha \beta}=\partial_{\alpha} \Gamma_{\mu \lambda \beta}-\partial_{\beta} \Gamma_{\mu \lambda \alpha} \text {. }
$$

Considérons le tenseur défini, relativement aux coordonnées adoptées, par:

$$
g_{\lambda, \mu}=\eta_{\lambda \mu}+\varepsilon \psi_{\lambda \mu \mu}
$$

où $\varepsilon$ est l'infiniment petit principal. Si nous effectuons le changement de coordonnées:

$$
x^{p^{\prime}}=x^{\rho}+\varepsilon \varphi p(x)
$$

on voit que $\psi_{\lambda_{1},}$ subit la transformation (dite encore de jauge)

$$
\psi_{\lambda_{\mu}} \rightarrow \psi_{\lambda \mu}+\partial^{\lambda} \varphi_{\mu}+\partial_{\mu} \varphi_{\lambda} .
$$

Nous pourons ainsi interpréter les résultats précedents en munissant l'espace-temps de la métrique riemanienne définie par $g_{\lambda \mu}$. En coordonnées voisines de coordonnes orthonormés par l'espace-temps minkowskien, cette métrique est voisine de la métrique minkowskienne definie par $\eta_{\lambda, k}$, la déviation subissant dans un changement de coordonnées la transformation (49.11) et $H_{\lambda \mu, \alpha \beta}$ est la partie principale du tenseur de courbure de $g_{\lambda, 1 .}$

$2^{\circ}$ ) Il nous reste à examiner les équations

$$
H_{\lambda, k}=0 \text {. }
$$

Par un choix convenable des $\varphi \rho$, on peut astreindre les $\Gamma_{\alpha \beta}^{2}$ aux conditions de coordonnées:

$$
\eta^{\alpha \beta} \Gamma_{\alpha \beta}^{\lambda}=0
$$

qui ne sont autres que la partie principale des conditions d'isothermie. On a alors:

$$
\eta^{\alpha \beta}\left(\partial_{\alpha} \psi_{\beta \mid \alpha}+\partial_{\beta} \psi_{\alpha \beta}-\partial_{\mu} \psi_{\alpha \beta}\right)=0
$$

et pour respecter (49-12), $\varphi_{\lambda}$ se trouve astreint anx conditions:

$$
\eta^{\alpha \beta} \partial_{\alpha \beta} \varphi_{\lambda}=0 .
$$

De (49.10), on déduit (voir (16-4)):

$$
H_{\lambda_{\mu}}=\frac{1}{2} \gamma_{i \beta}^{\alpha \beta}\left(\partial_{\alpha \lambda} \xi_{\beta_{k}}+\partial_{\beta \mu} \psi_{\alpha \lambda}-\partial_{\alpha \beta} \psi_{\lambda_{\mu}}-\partial_{\gamma_{\mu}} \psi_{\alpha \beta}\right)
$$

Les conditions de coordonnés, parties princirales des conditions d'isothermie, 
A. Lichnerowrcz: Ondes et radiations électromagnétiques, etc.

permettent de donner une expression simple de $H_{k, k}$. partie principale dn tenseur de Rrocr. De (49.13) on déduit par dérivation:

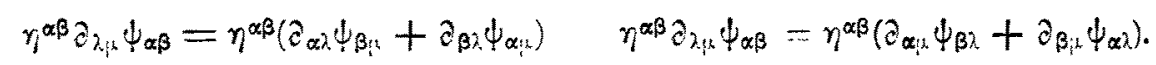

On en déduit que, sous les conditions de coordonnées

$$
H_{\lambda_{1 !}}=-\frac{1}{2} \eta^{\alpha \beta} \mathfrak{a}_{\alpha \beta} \psi_{\lambda_{\mu}}
$$

Ainsi, sous les conditions de coordonnées introduites, les équations $H_{\lambda_{\mu}}=0$ se traduisent par:

$$
\eta^{\alpha \beta} \partial_{\alpha \beta} \psi_{\lambda_{j \mu}}=0 .
$$

\section{Rapport avee la théorie du graviton.}

On peut montrer que notre processus est finmalement équivalent an processus classique qui conduit a la théorie du graviton; mais il se révèle plus cohérent et par suite plus satisfaisant mathématiquement et physiquement.

Sous les hypothèses simples permettant d'introduire la transformation de Fourier, on a en vertu de $(49.14)$ et $(49.15)$

$$
\psi_{\lambda_{, k}(\vec{x})}=\frac{1}{(2 \pi)^{3}} \int_{c} \chi_{\lambda, \mu} \overrightarrow{(l)} e^{i \vec{l} \cdot \vec{x}} d \Omega(\vec{l})
$$

et

$$
\left.\varphi_{i k}(\vec{x})=\frac{1}{(2 \pi)^{3}} \int_{c} f_{i t} \vec{l}\right) e^{i \vec{l} \cdot \vec{x}} d Q(\vec{l})
$$

où $C$ est le còne isotrope défini par $\eta_{\lambda, k} ; \psi_{\lambda, k}$ se trouvant défini à une transformation de jange près $\chi_{\lambda_{i}}$ se trouve défini à la transformation près :

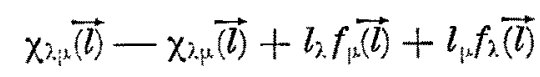

où $f_{i n}(\vec{l})$ est un vecteur arbitraire. On peut d'abord choisir ce vecteur de façon que

$$
\left.\chi \overrightarrow{(l)}=\eta^{\lambda_{1}} \chi_{\lambda, \mu} \vec{l} \vec{l}\right)=0
$$

et $f_{\xi}(\vec{l})$ est alors astreint a la condition:

$$
l f_{p} \overrightarrow{(l)}=0
$$

Si $\overrightarrow{n^{(1)}(\vec{l})} \overrightarrow{n^{(2)}(\vec{l})}$ sont encore denx vecteurs orthogonaux et normés tangents à $C$ le long de $\vec{l}, f_{i}(\vec{l})$ est un vecteur arbitraire de la forme:

$$
f_{\mu} \overrightarrow{(l)}=\frac{1}{2} \overrightarrow{a(\vec{l})} l_{\mu}+\sum_{i} b(i, \vec{l}) n_{k}^{(i)} \quad(i, j=1,2) .
$$


Pour que les conditions de coordonnées soient satisfaites, il faut et il suffit que:

$$
l^{2} \chi_{\lambda \mu} \vec{l} \vec{l}=0
$$

Il en résulte $q$ u'il existe des scalaires $a(\vec{l}), b(i, \vec{l}), c(i, j, \vec{l})$ (avec $c(i, j, \vec{l})=$ $=c(j, i, \vec{l})$ tels que:

$$
\chi_{\lambda \mu} \overrightarrow{(l)}=a(\vec{l}) l_{\lambda} l_{\mu}+\sum_{i} b(i, \vec{l})\left(l_{\lambda} n_{\mu^{2}}^{(i)}+l_{\mu} n_{\lambda}^{(i)}\right)+\sum_{i, j} c\left(i, j, \vec{l} n_{\lambda}^{(i)} n_{\mu}^{(j)}\right.
$$

En utilisant la transformation (50-1) avec (50-3), on voit que $\chi_{2, \mu}$ peut être astreint à la forme

$$
\chi_{\lambda \mu}(\vec{l})=\Sigma_{i, j} c(i, j, \vec{l}) n_{\lambda}^{(i)} n_{\mu}^{(j)}
$$

et est alors entièrement fixé; pour que $\chi(\vec{l})=0$, il faut et il suffit que l'on ait:

$$
c(1,1, \vec{l})+c(2,2, \vec{l})=0 .
$$

La quantification du champ $\psi_{\lambda \mu}$ s'effectuerà, selon notre processus, en imposant aux opérateurs substitués dans (50.4) aux scalaires $c(i, j, \vec{l})$ des conditions analogues à (47-8). Un caloul un peu long permet alors d'en déduice les relations de commutation (48-2).

\section{BIBLIOGRAPHIE}

L. BeL [1], *Comptes rendus Acad. Sc. Paris", t. 245, 2482 (1957).

-- [2], "Comptes rendus Acad. Sc. Paris", t. 248, 1297 (1959).

H. BoND1, * Nature», t. 179, 1072, (1957).

A. LICHNEROWICZ [1], Théories relativistes de la gravitation et de l'électromagnétisme, Paris (1955).

- [2], "Comptes rendus Acad. Sc. Paris s, t. 246, 893 (1958).

- - [3], a Comptes rendus Acad. Sc. Paris , t. 247, 433 (1958).

L. Mariot; Thése, Paris (1957).

A. Z. Petrov, sci. Not. de l'Université de Kazan», t. 114, p. 55 (1954).

F. A. E. Prrani [1], *Acta Phys. Polon. », t. 15, 389 (1956).

- [2], * Phys. Rev. s, t. 105, 1089 (1957).

N. Rosen, * Phys Z. Sorjet Union *, t. 12, 366 (1937).

K. Stellmacher, Math. Ann. *, t. 115, 740-783 (1988).

A. Trautman, Boundary conditions at infinity for physical theories - Radiation and Batu. dary conditions in the theory of gravitation, «Bull. Acad. Polon. Sci. s, t. 6 (1958). 